\title{
Twisted vertex algebras, bicharacter construction and boson-fermion correspondences
}

\author{
Iana I. Anguelova \\ Department of Mathematics, College of Charleston, Charleston SC 29424 *
}

\begin{abstract}
The boson-fermion correspondences are an important phenomena on the intersection of several areas in mathematical physics: representation theory, vertex algebras and conformal field theory, integrable systems, number theory, cohomology. Two such correspondences are well known: the types A and B (and their super extensions). As a main result of this paper we present a new bosonfermion correspondence, of type D-A. Further, we define a new concept of twisted vertex algebra of order $N$, which generalizes super vertex algebra. We develop the bicharacter construction which we use for constructing classes of examples of twisted vertex algebras, as well as for deriving formulas for the operator product expansions (OPEs), analytic continuations and normal ordered products. By using the underlying Hopf algebra structure we prove general bicharacter formulas for the vacuum expectation values for three important groups of examples. We show that the correspondences of type B, C and D-A are isomorphisms of twisted vertex algebras.
\end{abstract}

\section{Contents}

I. Introduction 2

A. Motivation 2

B. Overview of the paper

II. Notation and background 4

A. Notation 4

B. Super vertex algebras and the boson-fermion correspondence of type A 5

III. Twisted vertex algebras: definition, overview, examples 8

A. Twisted vertex algebras: definition and overview 8

B. Examples of twisted vertex algebras: the boson-fermion correspondence of type B 10

C. Examples of twisted vertex algebras: the boson-fermion correspondence of type D-A 12

D. Examples of twisted vertex algebras: the boson-fermion correspondence of type D-A of order $N \quad 13$

IV. Bicharacter construction: constructing examples of twisted vertex algebras 14

A. Super bicharacters and free Leibnitz modules 14

B. Examples of free Leibnitz modules $\quad 15$

C. Exponential map and its properties; Nonsingular twisted vertex algebras

D. Vertex operators, analytic continuations, OPEs and normal ordered products from a bicharacter 18

V. Examples of twisted vertex algebras based on a bicharacter 24

A. Twisted vertex algebras based on $\mathbb{C}\{\phi\}$ and a choice of a bicharacter $\quad 24$

B. Twisted vertex algebras based on $\mathbb{C}\{\phi\}$ : Pfaffian vacuum expectation values 24

C. Twisted vertex algebras based on $\mathbb{C}\{\phi\}$ : the neutral free fermion of type $\mathrm{B}$

D. Twisted vertex algebras based on $\mathbb{C}\{\phi\}$ : the neutral free fermion of type D-A $\quad 26$

E. Twisted vertex algebras based on $\mathbb{C}\{\phi, \psi\}$ : determinant vacuum expectation values

F. Super vertex algebra based on $\mathbb{C}\{\phi, \psi\}$ : the charged free fermions of type A 31

G. Twisted vertex algebras based on $\mathbb{C}[\mathbb{Z} \alpha]$ and a choice of a bicharacter $\quad 32$

H. Twisted vertex algebras based on $\mathbb{C}[\mathbb{Z} \alpha]$ : product vacuum expectation values

I. Super vertex algebra based on $\mathbb{C}[\mathbb{Z} \alpha]$ : the free boson of type A $\quad 33$

J. Twisted vertex algebra based on $\mathbb{C}[\mathbb{Z} \alpha]$ : the free boson of type $B \quad 33$

K. Twisted vertex algebra based on $\mathbb{C}[\mathbb{Z} \alpha]$ : the free boson of type D-A

L. Boson-fermion correspondence of type D-A and order $N$

*Electronic address: anguelovai@cofc.edu 
M. Other examples of twisted vertex algebras; the correspondence of type $\mathrm{C}$

\section{Summary}

References

\section{INTRODUCTION}

\section{A. Motivation}

In 2 dimensions the bosons and fermions are related by the boson-fermion correspondences. The best known boson-fermion correspondence is that of type A (see e.g. [Fre81], DJKM81a], KR87]). There are many properties and applications of this correspondence, often called the charged free fermion-boson correspondence, and an exposition of some of them is given in [KR87], [MJD00]. [DJKM81a] and [Fre81] discovered its connection to the theory of integrable systems (e.g. the KP and KdV hierarchies), to the theory of symmetric polynomials and the representation theory of the infinite dimensional Lie algebra $a_{\infty}$ (whence the name "type A" derives), as well as to $\hat{s l}_{n}$ and other affine Lie algebras. As this boson-fermion correspondence has so many applications and connections to various mathematical areas (including number theory and geometry, as well as random matrix theory and random processes), a deeper understanding of the types of mathematical structures that were being equated by it was sought. A partial answer early on was given by Igor Frenkel in [Fre81], but a complete answer had to wait for the development of the theory of vertex algebras. Vertex operators were introduced in string theory and now play an important role in many areas such as quantum field theory, integrable models, representation theory, random matrix theory, statistical physics, and many others. The theory of super vertex algebras axiomatizes the properties of some, simplest, "algebras" of vertex operators (see for instance [Bor86], FLM88], [FHL93], [Kac98], [LL04], [FBZ04]). Thus, as an application of the theory of super vertex algebras the question "what is the boson-fermion correspondence of type A?" can be answered precisely as follows: the boson-fermion correspondence of type A is an isomorphism of two super vertex algebras ( $\mathrm{Kac} 98]$ ).

There are other examples of boson-fermion correspondences, for instance the boson-fermion correspondence of type B (e.g. [DJKM82], You89], [vdLO09]), the super extensions of the boson-fermion correspondences of type A and B (KvdL89], Kac98]), and others ([JKM81b], voS11]). The first of those, the type B, was introduced in [DJKM82], and was discovered in connection to the theory of integrable systems, e.g. the BKP hierarchy ([DKM82]), and to the representation theory of the $b_{\infty}$ algebra (whence the name "type B" derives). Connections to the theory of symmetric polynomials and the symmetric group are shown in YYu89]. There is also ongoing work on other boson-fermion and boson-boson correspondences, e.g. the CKP correspondence (see [DJKM81b], voS11]).

Our motivation thus is twofold: first, we considered the questions "Are all boson-fermion correspondences isomorphisms of super vertex algebras? And if not, what are the boson-fermion correspondences, i.e., what mathematical structures do they equate?". And second, we considered the question "are there other bosonfermion correspondences?".

The first part of the first question is readily answered by the fact that in all other cases except the type A, the boson-fermion correspondences cannot be isomorphisms between two super vertex algebras, since the associated operator product expansions have singularities at both $z=w$ and $z=-w$. Hence, in order to answer the question, "what mathematical structures do these correspondences equate", we introduce the concept of a twisted vertex algebra of order $N$ (where $N$ is a positive integer). It generalizes the concept of a super vertex algebra in the sense that a super vertex algebra can be considered a twisted vertex algebra of order 1. The boson-fermion correspondence of type B is then an example of an isomorphism of twisted vertex algebras of order 2. There are other examples of isomorphisms of twisted vertex algebras in the literature: the correspondence of type CKP, which was introduced in [DJKM81b] and developed further in [vOS11], while strictly speaking not a boson-fermion correspondence since the generating operator product expansions are bosonic on both sides, is another such example, as is the super extension of the boson-fermion correspondence of type B.

As one of the main results of this paper, and as an answer to the second question posed, we introduce the new example of the boson-fermion correspondence of type D-A. This boson-fermion correspondence of type D-A completes the bosonisation of the 4 double-infinite rank affine Kac-Moody Lie algebras $a_{\infty}, b_{\infty}, c_{\infty}, d_{\infty}$. Although the super vertex algebra of the neutral fermion of type D is well known (see [Kac98], KWY98], KW94] ) as it gives the basic representation of $d_{\infty}$, this neutral fermion super vertex algebra does not itself 
have a bosonic equivalent, but as we show in this paper its twisted double cover does. We further show that it is another example of an isomorphism of twisted vertex algebras. Hence all the correspondences of type B, $\mathrm{C}$ and D-A are isomorphisms of twisted vertex algebras.

\section{B. Overview of the paper}

After detailing the notation and some background that we will use throughout the paper, we first briefly describe two examples of super vertex algebras. We assume the reader is familiar with the definition and properties of super vertex algebras, hence we directly list the relevant example of the boson-fermion correspondence of type A. We list only one property which is an "imprint" of the boson-fermion correspondence of type A: the Cauchy determinant identity which is a direct corollary of the equality between the vacuum expectation values of the two sides of the correspondence. (As discussed above, there are many properties and applications of any boson-fermion correspondence, which can, and do, occupy many papers). Next we proceed with the definition of a twisted vertex algebra (Section III). Twisted vertex algebras generalize super vertex algebras in two main directions. First, they have finitely many points of locality at $z=\epsilon^{i} w$, where $\epsilon$ is a primitive $N$ th root of unity (whereas super vertex algebras are only local at $z=w$ ). As such, twisted vertex algebras are similar to the $\Gamma$-vertex algebras of Li06], but are more general as they were designed to incorporate normal ordered products and operator product expansion coefficients as descendant fields (for a more detailed discussion on the comparison see [ACJ13]). The second main difference between twisted vertex algebras and super vertex algebras (and $\Gamma$-vertex algebras of [Li06]) is that in most of the examples of twisted vertex algebras the space of fields strictly contains the space of states. In fact a projection map from the space of fields to the space of states is part of the definition (see Definition III.1). In this aspect twisted vertex algebras more closely resemble the deformed chiral algebras defined in [FR97]. We do not discuss many properties of twisted vertex algebras in this paper due to length, instead we refer the interested readers to the follow-up paper [ACJ13]. In Section [II] we continue with the description of the examples of two pairs of twisted vertex algebras which constitute two examples of boson-fermion correspondences: the boson-fermion correspondence of type B (reformulated from [DJKM82] and You89] in the language of vertex algebras) and the boson-fermion correspondence of type D-A, which is new to this paper. We list only one property out of many for each correspondence, a representative identity which is a direct corollary of the equality between the vacuum expectation values of the two sides of the correspondence. For the correspondence of type B, this equality is actually the Schur Pfaffian identity (Lemma III.15), and for the type D-A it is a different Pfaffian identity (Lemma II.21). As the definition of a twisted vertex algebra is new and technical, its necessity can only be justified by some very meaningful examples of twisted vertex algebras - which indeed are the boson-fermion correspondences. Although there are many other examples of twisted vertex algebras, as we will see in theorem IV.41, the boson-fermion correspondences are important enough phenomena to justify this new definition of a twisted vertex algebra. Since the proofs of the statements in the narrative of the examples are lengthy enough to impede the overview, we give the proofs in the latter part of the paper, after we have introduced the requisite technical set of tools, namely the bicharacter construction. The bicharacter construction was first introduced in the context of vertex algebras in Bor01], the super bicharacters were introduced in Ang08]. The bicharacter construction is used in this paper in two ways. First, to give a general construction and description of variety of examples of twisted vertex algebras. We prove formulas for the analytic continuations, operator product expansions, vacuum expectation values using the bicharacters. (Such explicit formulas, for example the analytic continuations formulas, are very hard if not impossible to obtain otherwise even for specific examples.) Secondly, we use the bicharacter construction extensively to prove the properties of the new boson-fermion correspondence of type D-A.

There is a major difference between the bicharacter description of a vertex algebra and the operator-based description typically used: In the operator-based description the examples are presented in terms of generating fields (vertex operators) and their OPEs (or commutation relations). With the bicharacter construction one starts instead with a (supercommutative supercocommutative) Hopf algebra $M$ and its free Leibnitz module (for the definition and examples of free Leibnitz module see sections IV A and IV B). The commutation relations then result from the choice of a bicharacter $r$ - different bicharacter $r$ will dictate different commutation relations. Moreover, for each Hopf algebra $M$ there are many choices of a bicharacter $r$, hence each such pair $(M, r)$ will give rise to a different twisted vertex algebra, even if the underlying spaces of states are the same as Hopf algebras. It is in fact the field-state correspondence $Y$ that changes with each choice of a bicharacter, and hence the fields in the vertex algebra. This is the case for instance for the fermionic sides of both the B and D-A boson-fermion correspondences: they have identical spaces of states as Hopf algebras (which is not altogether surprising as they are both neutral fermions), but the collections of fields describing them differ 
substantially. Therefore in the bicharacter construction examples are grouped based on the underlying Hopf algebra $M$ - one starts by keeping $M$ the same, but changing the bicharacter $r$. We want to stress that there is a variety of examples even after we fix the algebra $M$. This description based on the Hopf algebra $M$ is further used to prove general formulas for the vacuum expectation values based on the Hopf algebra $M$ (sections $\mathrm{VB}, \mathrm{VE}$ and $\mathrm{VH}$ ). This is the explanation why the vacuum expectation values for both the B and the $\mathrm{D}$ fermion are Pfaffians, although, of course, different ones. In section $\mathrm{V}$ the particular examples of the fermionic and bosonic examples are detailed and their properties are proved, including the statements and assertions from sections $\amalg$ and $\amalg$ III

\section{NOTATION AND BACKGROUND}

\section{A. Notation}

In this section we list notations we will continuously use throughout the paper. Throughout we assume $N$ is a positive integer. We work over the field of complex numbers $\mathbb{C}$, with the category of super vector spaces, i.e., $\mathbb{Z}_{2}$ graded vector spaces. The flip map $\tau$ is defined by

$$
\tau(a \otimes b)=(-1)^{\tilde{a} \cdot \tilde{b}}(b \otimes a)
$$

for any homogeneous elements $a, b$ in the super vector space, where $\tilde{a}, \tilde{b}$ denote correspondingly the parity of $a, b$.

A superbialgebra $H$ is a an associative algebra with a $\mathbb{Z}_{2}$ grading and a compatible coalgebra structure, in the sense that the coproduct denoted by $\triangle$ and the counit denoted by $\eta$ are algebra maps. A Hopf superalgebra is a superbialgebra with an antipode $S$. For a superbialgebra $H$ we will write $\triangle(a)=\sum a^{\prime} \otimes a^{\prime \prime}$ for the coproduct of $a \in H$ (Sweedler's notation); i.e., we will usually omit the indexing in $\triangle(a)=\sum_{k} a_{k}^{\prime} \otimes a_{k}^{\prime \prime}$, especially when it is clear from the context. Recall in a super Hopf algebra the product on $H \otimes H$ is defined by

$$
(a \otimes b)(c \otimes d)=(-1)^{\tilde{b} \cdot \tilde{c}}(a c \otimes b d)
$$

for any $a, b, c, d$ homogeneous elements in $H$. A supercocommutative bialgebra is a superbialgebra with $\tau(\triangle(a))=\triangle(a)$. If $H$ is a Hopf super algebra, an element $a \in H$ satisfying $\triangle(a)=a \otimes 1+1 \otimes a, \eta(a)=0$, and $S(a)=-a$ is called "primitive" A element $g \in H$ satisfying $\triangle(g)=g \otimes g, \eta(g)=1, S(g)=g^{-1}$ is called "grouplike".

Notation II.1 For any $a \in A$, where $A$ is a commutative associative $\mathbb{C}$ algebra denote $a^{(n)}:=\frac{a^{n}}{n !}$.

Definition II.2 (The Hopf algebra $H_{D}=\mathbb{C}[D]$ )

The Hopf algebra $H_{D}=\mathbb{C}[D]$ is the polynomial algebra with a primitive generator $D$. We have

$$
\triangle D^{(n)}=\sum_{k+l=n} D^{(k)} \otimes D^{(l)} .
$$

\section{Definition II.3 (The Hopf algebra $H_{T_{\epsilon}}^{N}$ )}

Let $\epsilon$ be a primitive root of unity of order $N$. The Hopf algebra $H_{T_{\epsilon}}^{N}$ is the Hopf algebra with a primitive generator $D$ and a grouplike generator $T_{\epsilon}$ subject to the relations:

$$
D T_{\epsilon}=\epsilon T_{\epsilon} D, \quad\left(T_{\epsilon}\right)^{N}=1 .
$$

$H_{T_{\epsilon}}^{N}$ contains $H_{D}$ as a Hopf subalgebra. Both $H_{D}$ and $H_{T_{\epsilon}}^{N}$ are entirely even.

Notation II.4 (The function spaces $\left.\mathbf{F}^{N}(z, w), \mathbf{F}^{N}(z, w)^{+, w}\right)$

Let $\epsilon$ be a primitive root of unity of order $N$. Denote by $\mathbf{F}^{N}(z, w)$ the space of rational functions in the formal variables $z, w$ with only poles at $z=0, w=0, z=\epsilon^{i} w, i=1, \ldots, N$ :

$$
\mathbf{F}^{N}(z, w)=\mathbb{C}[z, w]\left[z^{-1}, w^{-1},(z-w)^{-1},(z-\epsilon w)^{-1}, \cdots,\left(z-\epsilon^{N-1} w\right)^{-1}\right] .
$$

Also, denote $\mathbf{F}^{N}(z, w)^{+, w}$ the space of rational functions in the formal variables $z, w$ with only poles at $z=$ $0, z=\epsilon^{i} w, i=1, \ldots, N$. Note that we do not allow a pole at $w=0$ in $\mathbf{F}^{N}(z, w)^{+, w}$, i.e., if $f(z, w) \in \mathbf{F}^{N}(z, w)$, 
then $f(z, 0)$ is well defined. More generally, let $\mathbf{F}^{N}\left(z_{1}, z_{2}, \ldots, z_{l}\right)$ is the space of rational functions in variables $z_{1}, z_{2}, \ldots, z_{l}$ with only poles at $z_{m}=0, m=1, \ldots, l$, or at $z_{i}=\epsilon^{k} z_{j}$, and $i \neq j=1, \ldots, l, k=1, \ldots, N$. Lastly $\mathbf{F}^{N}\left(z_{1}, z_{2}, \ldots, z_{l}\right)^{+, z_{l}}$ is the space of rational functions in variables $z_{1}, z_{2}, \ldots, z_{l}$ with only poles at $z_{m}=0$, $m=1, \ldots, l-1$, or at $z_{i}=\epsilon^{k} z_{j}, i \neq j=1, \ldots, l, k=1, \ldots, N$.

Fact II.5 $\mathbf{F}^{N}(z, w)$ is an $H_{T_{\epsilon}}^{N} \otimes H_{T_{\epsilon}}^{N}$ (and consequently an $H_{D} \otimes H_{D}$ ) Hopf module by

$$
\begin{aligned}
& D_{z} f(z, w)=\partial_{z} f(z, w), \quad\left(T_{\epsilon}\right)_{z} f(z, w)=f(\epsilon z, w) \\
& D_{w} f(z, w)=\partial_{w} f(z, w), \quad\left(T_{\epsilon}\right)_{w} f(z, w)=f(z, \epsilon w)
\end{aligned}
$$

We will denote the action of elements $h \otimes 1 \in H_{T_{\epsilon}}^{N} \otimes H_{T_{\epsilon}}^{N}$ on $\mathbf{F}^{N}(z, w)$ by $h_{z}$, correspondingly $h_{w} \cdot$ will denote the action of the elements $1 \otimes h \in H_{T_{\epsilon}}^{N} \otimes H_{T_{\epsilon}}^{N}$.

Notation II.6 For a rational function $f(z, w)$ we denote by $i_{z, w} f(z, w)$ the expansion of $f(z, w)$ in the region $|z| \gg|w|$, and correspondingly for $i_{w, z} f(z, w)$. Similarly, we will denote by $i_{z_{1}, z_{2}, \ldots, z_{n}}$ the expansion in the region $\left|z_{1}\right| \gg \cdots \gg\left|z_{n}\right|$.

\section{B. Super vertex algebras and the boson-fermion correspondence of type A}

The following definitions are well known (see for instance [FLM88], [FHL93], [Kac98], [LL04] and others).

Definition II.7 (Field) A field $a(z)$ on a vector space $V$ is a series of the form

$$
a(z)=\sum_{n \in \mathbf{Z}} a_{(n)} z^{-n-1}, \quad a_{(n)} \in \operatorname{End}(V), \text { such that } a_{(n)} v=0 \quad \forall v \in V, n \gg 0 .
$$

Remark II.8 The coefficients $a_{(n)}, n \in \mathbb{Z}$, are often called modes. The indexing above is typically used in super vertex algebras: if a field $a(z)$ with this indexing is a vertex operator in a super vertex algebra, then the modes $a_{(n)}$ with $n \geq 0$ annihilate the vacuum vector (hence are called annihilation operators), and the modes $a_{(n)}$ with $n<0$ are the creation operators. Denote

$$
a(z)_{-}:=\sum_{n \geq 0} a_{n} z^{-n-1}, \quad a(z)_{+}:=\sum_{n<0} a_{n} z^{-n-1} .
$$

Definition II.9 (Normal ordered product) Let $a(z), b(z)$ be fields on a vector space $V$. Define the normal ordered product of these fields by

$$
: a(z) b(w):=a(z)_{+} b(w)+(-1)^{\tilde{a} \tilde{b}} b(w) a_{-}(z) .
$$

Remark II.10 Let $a(z), b(z) \in \operatorname{End}(V)\left[\left[z^{ \pm 1}\right]\right]$ be fields on a vector space $V$. Then $: a(z) b(\lambda z):$ and $: a(\lambda z) b(z)$ : for any $\lambda \in \mathbb{C}^{*}$ are well defined elements of $\operatorname{End}(V)\left[\left[z^{ \pm 1}\right]\right]$, and are also fields on $V$.

Remark II.11 The space of fields on a vector space $V$ is an $H_{T_{\epsilon}}^{N}$ module via the same action $h_{z}$. used on the space $\mathbf{F}^{N}(z, w)$.

The definition of a super vertex algebra is well known, we refer the reader for example to [FLM88], [FHL93], Kac98], LL04], as well as for notations, details and theorems. Super vertex algebras have two important properties which we would like to carry over to the case of twisted vertex algebras. These are the properties of analytic continuation and completeness with respect to operator product expansions (OPEs). In fact our definition of a twisted vertex algebra is based on enforcing these two properties. Recall we have for the OPE of two local (at $z=w$ ) fields (see e.g. Kac98])

$$
a(z) b(w)=\sum_{j=0}^{N-1} i_{z, w} \frac{c^{j}(w)}{(z-w)^{j+1}}+: a(z) b(w): .
$$

Moreover, if the two fields $a(z), b(w)$ are vertex operators in a super vertex algebra we have $\operatorname{Res}_{(z-w)} a(z) b(w)(z-w)^{j}=c^{j}(w)=\left(a_{(j)} b\right)(w)$, i.e., the coefficients of the OPEs are vertex operators in 
the same super vertex algebra (i.e., attached to elements of the vertex algebra). Since the commutation relations are determined only by the singular part of the OPE, we abbreviate the OPE above as:

$$
a(z) b(w) \sim \sum_{j=0}^{N-1} \frac{c^{j}(w)}{(z-w)^{j+1}}
$$

Also, an analytic continuation property for a super vertex algebra holds: for any $a_{i} \in V, i=1, \ldots, k$, there exist a rational vector valued function

$$
X_{z_{1}, z_{2}, \ldots, z_{k}}: V^{\otimes k} \rightarrow W\left[\left[z_{1}, z_{2}, \ldots, z_{k}\right]\right] \otimes \mathbf{F}_{\epsilon}^{1}\left(z_{1}, z_{2}, \ldots, z_{k}\right)^{+, z_{k}}
$$

such that

$$
Y\left(a_{1}, z_{1}\right) Y\left(a_{2}, z_{2}\right) \ldots Y\left(a_{k}, z_{k}\right) 1=i_{z_{1}, z_{2}, \ldots, z_{k}} X_{z_{1}, z_{2}, \ldots, z_{k}}\left(a_{1} \otimes a_{2} \otimes \ldots \otimes a_{k}\right) .
$$

For many examples, super vertex algebras are generated by a much smaller number of generating fields (see e.g. Kac98]), with imposing the condition that the resulting space of fields of the vertex algebra has to be closed under certain operations: for any vertex operator $a(z)$ the field $D a(z)=\partial_{z} a(z)$ has to also be a vertex operator in the vertex algebra. Also, the OPEs coefficients $\left(c^{j}(w)\right.$ from (II.11) ) and normal ordered products $: a(z) b(z)$ : of any two vertex operators $a(z)$ and $b(w)$ have to be vertex operators in the vertex algebra. Note that the identity operator on $V$ is always a vertex operator in the vertex algebra corresponding to the vacuum vector $|0\rangle \in V$.

Next we briefly recall the boson-fermion correspondence of type A, which is an isomorphism of super vertex algebras. The fermion side of the boson-fermion correspondence of type A is a super vertex algebra generated by two nontrivial odd fields - two charged fermions: the fields $\phi(z)$ and $\psi(z)$ with only nontrivial operator product expansion (OPE):

$$
\phi(z) \psi(w) \sim \frac{1}{z-w} \sim \psi(z) \phi(w), \quad \phi(z) \phi(w) \sim 0 \sim \psi(z) \psi(w),
$$

where the 1 above denotes the identity map $I d$. The modes $\phi_{n}$ and $\psi_{n}, n \in \mathbb{Z}$, of the fields $\phi(z)$ and $\psi(z)$, which we index as follows:

$$
\phi(z)=\sum_{n \in \mathbf{Z}} \phi_{n} z^{n}, \quad \psi(z)=\sum_{n \in \mathbf{Z}} \psi_{n} z^{n},
$$

form a Clifford algebra $C l_{A}$ with relations

$$
\left[\phi_{m}, \psi_{n}\right]_{\dagger}=\delta_{m+n,-1} 1, \quad\left[\phi_{m}, \phi_{n}\right]_{\dagger}=\left[\psi_{m}, \psi_{n}\right]_{\dagger}=0 .
$$

The indexing of the generating fields vary depending on the point of view; our indexing here corresponds to $\phi_{n}=\hat{v}_{n+1}, \quad \psi_{n}=\check{v}_{-n}^{*}$ of [KR87]. Here our choice corresponds to our bicharacter description of this example. This indexing and the properties of the vertex algebra dictate that the underlying space of states of this super vertex algebra - the fermionic Fock space, denoted by $F_{A}$ - is the highest weight representation of $C l_{A}$ generated by the vacuum vector $|0\rangle$, so that $\phi_{n}|0\rangle=\psi_{n}|0\rangle=0$ for $n<0$. We denote by $F_{A}$ both the space of states and the resulting vertex algebra generated by the fields $\phi(z)$ and $\psi(z)$; it is often called the charged free fermion vertex algebra.

We can calculate vacuum expectation values if we have a symmetric bilinear form $\langle\mid\rangle: V \otimes V \rightarrow \mathbb{C}$ on the space of states of the vertex algebra $V$. There is a very important concept of an invariant bilinear form on a vertex algebra, for details see for example [FHL93], [Li94]. We will not recall the full definition here, but we will require that the bilinear form is such that the vacuum vector $|0\rangle$ spans an orthogonal subspace on its own (for a precise statement in the context of the bicharacter construction see sections VB and VE). We also require that the bilinear form is normalized on the vacuum vector:

$$
\langle\langle 0|| \mid 0\rangle\rangle=1 \text {. }
$$

By abuse of notation we will just write $\langle 0 \mid 0\rangle$ instead of $\langle\langle 0|| \mid 0\rangle\rangle$.

Lemma II.12 The following determinant formula for the vacuum expectation values on the fermionic side $F_{A}$ holds:

$$
\left\langle 0\left|\phi\left(z_{1}\right) \phi\left(z_{2}\right) \ldots \phi\left(z_{n}\right) \psi\left(w_{1}\right) \psi\left(w_{2}\right) \ldots \psi\left(w_{n}\right)\right| 0\right\rangle=(-1)^{n(n-1) / 2} i_{z, w} \operatorname{det}\left(\frac{1}{z_{i}-w_{j}}\right)_{i, j=1}^{n} .
$$

Here $i_{z ; w}$ stands for the expansion $i_{z_{1}, z_{2}, \ldots, z_{n}, w_{1}, \ldots, w_{n}}$. 
This formula is well known, new proof as a corollary of the bicharacter description will be given in the section $\mathrm{VF}$

The boson-fermion correspondence of type A is determined once we write the images of generating fields $\phi(z)$ and $\psi(z)$ under the correspondence. In order to do that, an essential ingredient is the so-called Heisenberg field $h(z)$ given by

$$
h(z)=: \phi(z) \psi(z):
$$

It follows that the Heisenberg field $h(z)=\sum_{n \in \mathbb{Z}} h_{n} z^{-n-1}$ has OPEs with itself given by:

$$
h(z) h(w) \sim \frac{1}{(z-w)^{2}}, \quad \text { in modes: }\left[h_{m}, h_{n}\right]=m \delta_{m+n, 0} 1 .
$$

i.e., its modes $h_{n}, n \in \mathbb{Z}$, generate a Heisenberg algebra $\mathcal{H}_{\mathbb{Z}}$ (which is the reason for the name Heisenberg field). It is well known that any irreducible highest weight module of this Heisenberg algebra is isomorphic as Heisenberg module to the polynomial algebra $B_{m} \cong \mathbb{C}\left[x_{1}, x_{2}, \ldots, x_{n}, \ldots\right]$ with countably many variables via the action:

$$
h_{n} \mapsto \partial_{x_{n}}, \quad h_{-n} \mapsto n x_{n}, \quad \text { for any } n \in \mathbb{N}, \quad h_{0} \mapsto m \cdot .
$$

The fermionic Fock space decomposes (via the charge decomposition, for details see for example [KR87]) as $F_{A}=\oplus_{i \in \mathbb{Z}} B_{i}$, which we can write as

$$
F_{A}=\oplus_{i \in \mathbb{Z}} B_{i} \cong \mathbb{C}\left[e^{\alpha}, e^{-\alpha}\right] \otimes \mathbb{C}\left[x_{1}, x_{2}, \ldots, x_{n}, \ldots\right],
$$

where by $\mathbb{C}\left[e^{\alpha}, e^{-\alpha}\right]$ we mean the Laurent polynomials with one variable $e^{\alpha}$ (the reason for this notation is that the resulting vertex algebra on the right hand side is a lattice vertex algebra.) The isomorphism is as Heisenberg modules, where $e^{n \alpha}$ identifies the highest weight vector for the irreducible Heisenberg module $B_{n}$. We denote the vector space on the right-hand-side of this $\mathcal{H}_{\mathbb{Z}}$-module isomorphism by $B_{A}:=\mathbb{C}\left[e^{\alpha}, e^{-\alpha}\right] \otimes \mathbb{C}\left[x_{1}, x_{2}, \ldots, x_{n}, \ldots\right] . B_{A}$ is the underlying vector space of the bosonic side of the bosonfermion correspondence of type A.

Now we can write the images of generating fields $\phi(z)$ and $\psi(z)$ under the correspondence:

$$
\phi(z) \mapsto e_{A}^{\alpha}(z), \quad \psi(z) \mapsto e_{A}^{-\alpha}(z),
$$

where the generating fields $e_{A}^{\alpha}(z), e_{A}^{-\alpha}(z)$ for the bosonic part of the correspondence are given by

$$
\begin{aligned}
e_{A}^{\alpha}(z) & =\exp \left(\sum_{n \geq 1} \frac{h_{-n}}{n} z^{n}\right) \exp \left(-\sum_{n \geq 1} \frac{h_{n}}{n} z^{-n}\right) e^{\alpha} z^{\partial_{\alpha}}, \\
e_{A}^{-\alpha}(z) & =\exp \left(-\sum_{n \geq 1} \frac{h_{-n}}{n} z^{n}\right) \exp \left(\sum_{n \geq 1} \frac{h_{n}}{n} z^{-n}\right) e^{-\alpha} z^{-\partial_{\alpha}},
\end{aligned}
$$

the operators $e^{\alpha}, e^{-\alpha}, z^{\partial_{\alpha}}$ and $z^{-\partial_{\alpha}}$ act in an obvious way on the space $B_{A}$.

The resulting super vertex algebra generated by the fields $e_{A}^{\alpha}(z)$ and $e_{A}^{-\alpha}(z)$ acting on the underlying vector space $B_{A}$ we denote also by $B_{A}$.

Lemma II.13 The following product formula for the vacuum expectation values on the bosonic side $B_{A}$ holds:

$$
\left\langle 0\left|e_{A}^{\alpha}\left(z_{1}\right) e_{A}^{\alpha}\left(z_{2}\right) \ldots e_{A}^{\alpha}\left(z_{n}\right) e_{A}^{-\alpha}\left(w_{1}\right) e_{A}^{-\alpha}\left(w_{2}\right) \ldots e_{A}^{-\alpha}\left(w_{n}\right)\right| 0\right\rangle=i_{z, w} \frac{\prod_{i<j}^{n}\left(\left(z_{i}-z_{j}\right)\left(w_{i}-w_{j}\right)\right)}{\prod_{i, j=1}^{n}\left(z_{i}-w_{j}\right)}
$$

Here $i_{z, w}$ stands for the expansion $i_{z_{1}, z_{2}, \ldots, z_{n}, w_{1}, \ldots, w_{n}}$.

The proof will be given in the section VI.

Theorem II.14 ([Kac98]) The boson-fermion correspondence of type $A$ is the isomorphism between the charged free fermions super vertex algebra $F_{A}$ and the bosonic super vertex algebra $B_{A}$. 
Corollary II.15 The Cauchy's determinant identity follows from the equality of the vacuum expectation values (AC stands for Analytic Continuation):

$$
\begin{aligned}
(-1)^{n(n-1) / 2} \operatorname{det}\left(\frac{1}{z_{i}-w_{j}}\right)_{i, j=1}^{n} & =A C\left\langle 0\left|\phi\left(z_{1}\right) \ldots \phi\left(z_{n}\right) \psi\left(w_{1}\right) \ldots \psi\left(w_{n}\right)\right| 0\right\rangle \\
& =A C\left\langle 0\left|e_{A}^{\alpha}\left(z_{1}\right) \ldots e_{A}^{\alpha}\left(z_{n}\right) e_{A}^{-\alpha}\left(w_{1}\right) \ldots e_{A}^{-\alpha}\left(w_{n}\right)\right| 0\right\rangle=\frac{\prod_{i<j}\left(\left(z_{i}-z_{j}\right)\left(w_{i}-w_{j}\right)\right)}{\prod_{i, j=1}^{n}\left(z_{i}-w_{j}\right)} .
\end{aligned}
$$

It is important to note that although this determinant identity is obviously well known and has been proved in a variety of ways since Cauchy, it is also a direct corollary of the boson-fermion correspondence of type A.

\section{TWISTED VERTEX ALGEBRAS: DEFINITION, OVERVIEW, EXAMPLES}

\section{A. Twisted vertex algebras: definition and overview}

Definition III.1 (Twisted vertex algebra of order $N$ ) A twisted vertex algebra of order $N$ is a collection $(V, W, \pi, Y)$ of the following data:

- the space of fields $V$ : a vector super space and an $H_{T_{\epsilon}}^{N}$ module graded as an $H_{D}$-module;

- the space of states $W$ : a vector super subspace of $V$;

- a linear surjective projection map $\pi: V \rightarrow W$, such that $\left.\pi\right|_{W}=I d_{W}$

- a field-state correspondence $Y$ : a linear map from $V$ to the space of fields on $W$;

- a vacuum vector: a vector $|0\rangle \in W \subset V$.

This data should satisfy the following set of axioms:

- vacuum axiom: $Y(|0\rangle, z)=I d_{W}$;

- modified creation axiom: $\left.Y(a, z)|0\rangle\right|_{z=0}=\pi(a)$, for any $a \in V$;

- transfer of action: $Y(h a, z)=h_{z} \cdot Y(a, z)$ for any $h \in H_{T_{\epsilon}}^{N}$;

- analytic continuation: For any $a, b, c \in V$ exists $X_{z, w, 0}(a \otimes b \otimes c) \in W[[z, w]] \otimes \mathbf{F}^{N}(z, w)$ such that

$$
Y(a, z) Y(b, w) \pi(c)=i_{z, w} X_{z, w, 0}(a \otimes b \otimes c)
$$

- symmetry: $X_{z, w, 0}(a \otimes b \otimes c)=X_{w, z, 0}(\tau(a \otimes b) \otimes c)$;

- Completeness with respect to Operator Product Expansions (OPE's): For each $i \in 0,1, \ldots, N-1$, $k \in \mathbb{Z}$, and any $a, b, c \in V$, where $a, b$ are homogeneous with respect to the grading by $D$, there exist $l_{k, i}^{s} \in \mathbb{Z},\left|l_{k, i}^{s}\right| \leq(N-1)(k+1)$, such that

$$
\operatorname{Res}_{z=\epsilon^{i} w} X_{z, w, 0}(a \otimes b \otimes c)\left(z-\epsilon^{i} w\right)^{k}=\sum_{s}^{\text {finite }} w^{l_{k, i}^{s}} Y\left(v_{k, i}^{s}, w\right) \pi(c)
$$

for some homogeneous elements $v_{k, i}^{s} \in V, \quad l_{k, i}^{s} \in \mathbb{Z}$.

Remark III.2 If $V$ is an (ordinary) super vertex algebra, then the data $\left(V, V, \pi=I d_{V}, Y\right)$ is a twisted vertex algebra of order 1. Moreover, it can be proved that a twisted vertex algebra $\left(V, V, \pi=I d_{V}, Y\right)$ of order 1 is a super vertex algebra, due to the restrictions $\left|l_{k, i}^{s}\right| \leq(N-1)(k+1)$ in the shifts $l_{k, i}^{s}$.

The definition of a twisted vertex algebra is very similar to the definition of a deformed chiral algebra given by E. Frenkel and Reshetikhin in [FR97]. 
Remark III.3 (OPE shifts) The axiom requiring completeness with respect to the OPEs is weaker than the OPE property of super vertex algebras. We can express this weaker axiom as follows (for more details see [ACJ13]): the OPE coefficient, the residue $\operatorname{Res}_{z=\epsilon^{i} w} X_{z, w, 0}(a \otimes b \otimes c)\left(z-\epsilon^{i} w\right)^{k}$, gives the values of a field $v(w)$ which may not be a vertex operator associated to an element of $V$. But each mode $v_{(n)}$ is a finite sum of the modes $v_{k, i}^{s}(w)_{\left(n-l_{k, i}^{s}\right)}$ of the corresponding fields $v_{k, i}^{s}(w)$. Each of these modes $v_{k, i}^{s}(w)_{\left(n-l_{k, i}\right)}$ may require a different shift $l_{k, i}^{s}$ in its index. As was mentioned in the previous section, in a super vertex algebra we have a stronger property, requiring that the OPE coefficients automatically, without need of a shift, be vertex operators in the same vertex algebra (i.e., attached to an element of $V$ ). This stronger property cannot hold in the interesting examples, which forced the modification of the OPE completeness axiom (see remark III.7 below).

Definition III.4 (Isomorphism of twisted vertex algebras) Two twisted vertex algebras $(V, W, \pi, Y)$ and $\left(\widetilde{V}, \widetilde{W}, \tilde{\pi}_{f}, \widetilde{Y}\right)$ are said to be isomorphic via a linear bijective map $\Phi: V \rightarrow \widetilde{V}$ if $\Phi\left(|0\rangle_{W}\right)=|0\rangle_{\widetilde{W}}$ and the following holds: for any $v \in V, \tilde{v} \in \widetilde{V}$ and $w \in W, \tilde{w} \in \widetilde{W}$ we have

$$
\Phi(v)=\sum_{\text {finite }} c_{k} \tilde{v}_{k}, \quad c_{k} \in \mathbb{C}, \quad \tilde{v}_{k} \in \tilde{V}, \quad \Phi^{-1}(\tilde{v})=\sum_{\text {finite }} d_{m} v_{m}, \quad d_{m} \in \mathbb{C}, \quad v_{m} \in V ;
$$

so that

$$
\begin{aligned}
& \Phi(Y(v, z) w)=\sum_{\text {finite }} z^{l_{k}} c_{k} \tilde{Y}\left(\tilde{v}_{k}, z\right) \tilde{\pi}_{f} \circ \Phi(w), \quad l_{k} \in \mathbb{Z} ; \\
& \Phi^{-1}(\tilde{Y}(\tilde{v}, z) \tilde{w})=\sum_{\text {finite }} z^{l_{m}} d_{m} Y\left(v_{m}, z\right) \pi \circ \Phi^{-1}(\tilde{w}), \quad l_{m} \in \mathbb{Z} .
\end{aligned}
$$

Remark III.5 This definition is much more complicated than in the super vertex algebra case due to the allowance for the shifts in the OPEs. This can cause each of the summands in the linear sum $\Phi(v)$ to appear with a different shift in the sum of the corresponding vertex operators, e.g. (V.38) and (V.52), hence the allowance for the different powers of $z$ in the above definition.

There are a variety of different vertex algebra like theories, each designed to describe different sets of examples of collections of fields. The best known is the theory of super vertex algebras (see e.g. [FLM88], Kac98], LL04], Bor86], [FBZ04]). The axioms of super vertex algebras are often given in terms of locality (see Kac98], [FBZ04]), as locality is a property that plays crucial importance for super vertex algebras ([Li96]). On the other hand, there are vertex algebra like objects which do not satisfy the usual locality property, but rather a generalization. Twisted vertex algebras are among them, but there are also generalized vertex algebras, $\Gamma$-vertex algebras, deformed chiral algebras, quantum vertex algebras. Twisted vertex algebras occupy intermediate step between super vertex algebras and deformed chiral algebras of [FR97]). We chose here to define twisted vertex algebras with axioms closer to the deformed chiral algebra axioms, including the analytic continuation axiom. But twisted vertex algebras are closer to super vertex algebras in two aspects. First, they satisfy $N$-point locality (finitely many points of locality), see ACJ13], unlike the deformed chiral algebras which have lattices of points of locality. Second, as for super vertex algebras, the axioms requiring existence of analytic continuation of product of two vertex operators plus the symmetry axiom do in fact enforce the property that analytic continuation of arbitrary product of fields exist; something that is not true for deformed chiral algebras (see [FR97]). This property, which we will not prove here (proof is given in [ACJ13]), is why we chose to give the axioms for twisted vertex algebra in the form above. Further, we will derive formulas for the analytic continuations using the bicharacter construction which we will use to derive vacuum expectation values identities.

Proposition III.6 (Analytic continuation for arbitrary products of fields)

Let $(V, W, \pi, Y)$ be a twisted vertex algebra. There exists a rational vector valued function

$$
X_{z_{1}, z_{2}, \ldots, z_{k}}: V^{\otimes k} \rightarrow W\left[\left[z_{1}, z_{2}, \ldots, z_{k}\right]\right] \otimes \mathbf{F}^{N}\left(z_{1}, z_{2}, \ldots, z_{k}\right)^{+, z_{k}},
$$

such that

$$
Y\left(a_{1}, z_{1}\right) Y\left(a_{2}, z_{2}\right) \ldots Y\left(a_{k}, z_{k}\right) 1=i_{z_{1}, z_{2}, \ldots, z_{k}} X_{z_{1}, z_{2}, \ldots, z_{k}}\left(a_{1} \otimes a_{2} \otimes \ldots \otimes a_{k}\right)
$$

for any $a_{i} \in V, i=1, \ldots, k$. 
We do not discuss the axiomatics of twisted vertex algebras further in this paper due to length (for this see [ACJ13]). Instead we show that the concept of a twisted vertex algebra answers the question "what mathematical structures are the boson-fermion correspondences of type B, C and D-A?". We claim that these boson-fermion correspondences are isomorphisms of twisted vertex algebras.

Similarly to super vertex algebras, twisted vertex algebras are often generated by a smaller number of fields. We will not prove theorems on what constitutes a generating set of fields (see [ACJ13]), instead we will take an alternative approach and use the bicharacter construction which we will present in the next section. The bicharacter construction in some sense mimics the generation by a smaller set of generating fields, in that we start with a smaller set of data which then "generates" the entire set of data for the twisted vertex algebra. The bicharacter construction will thus replace the necessary theorems on generating sets of fields. We want to mention though, that if we have a set of generating fields, the full space of fields is in turn determined by requiring, as in a super vertex algebra, that it be closed under OPEs (see modification above); that for any vertex operator $a(z)$ the field $D a(z)=\partial_{z} a(z)$ has to be a vertex operator in the twisted vertex algebra. A new ingredient in a twisted vertex algebra is the requirement that the field $T_{\epsilon} a(z)=a(\epsilon z)$ is a vertex operator in the same twisted vertex algebra as well. Note that this immediately violates the stronger creation axiom for a classical vertex algebra, since:

$$
\left.\left(T_{\epsilon} a\right)(z)|0\rangle\right|_{z=0}=\left.a(\epsilon z)|0\rangle\right|_{z=0}=\left.a(z)|0\rangle\right|_{z=0}=\pi(a) .
$$

Hence any such field $T_{\epsilon} a(z)$ cannot belong to a classical vertex algebra. This is the reason we require the projection map $\pi$ to be a part of our data for a twisted vertex algebra, as well as we modify the field-state correspondence with the modified creation axiom. The projection map $\pi$ in the definition of a twisted vertex algebra $(V, W, \pi, Y)$ could be made more general, for example one can omit the requirement that $W \subset V$ and make $\pi$ a more general linear surjective projection map, but the current generality is sufficient for the examples we want to describe.

\section{B. Examples of twisted vertex algebras: the boson-fermion correspondence of type B}

We now proceed with the two examples of a twisted vertex algebra of order 2 which give the two sides of the boson-fermion correspondence of type B. This correspondence was first introduced in DJKM82, and was interpreted as an isomorphism of twisted vertex algebras in Ang13 (without proofs). Since this is a case of twisted vertex algebras of order two, the root of unity is -1 , and we will write $T_{-1}$ instead of $T_{\epsilon}$. The proofs of all the statements and assertions in this section will be given in sections $\mathrm{VC}$ and $\mathrm{VJ}$.

The fermionic side is described via a single field $\phi^{B}(z)=\sum_{n \in \mathbf{Z}} \phi_{n}^{B} z^{n}$, with OPE given by:

$$
\phi^{B}(z) \phi^{B}(w) \sim \frac{-2 w}{z+w}, \quad \text { in modes: }\left[\phi_{m}^{B}, \phi_{n}^{B}\right]_{\dagger}=2(-1)^{m} \delta_{m,-n} 1 .
$$

The modes generate a Clifford algebra $C l_{B}$, and the underlying space of states, denoted by $F_{B}$, of the twisted vertex algebra is a highest weight representation of $C l_{B}$ with the vacuum vector $|0\rangle$, such that $\phi_{n}^{B}|0\rangle=$ 0 for $n<0$. The space of fields, which is larger than the space of states, is generated via the field $\phi^{B}(z)$ together with its descendent $T_{-1} \phi^{B}(z)=\phi^{B}(-z)$. We will prove in Section VC that there exists a twisted vertex algebra, denoted by $T V A\left(F_{B}\right)$, with a space of states $F_{B}$.

Remark III.7 The defining OPE, (III.6), shows why we adopted the modification of the completeness with respect to OPEs. The field defined by the residue at $z=-w$ in (III.6) is $c(w):=-2 w I d_{W}$. Note that $c(w)=-2 w I d_{W}$ is a field, but is not a vertex operator in a super or twisted vertex algebra as is. But a shift by $w^{-1}$ will produce the field $w \cdot c(w)=-2 I d_{W}$, which is the vertex operator $Y(-2|0\rangle, w)$ attached to the state $-2|0\rangle$.

Remark III.8 One often writes, especially in physics, the OPE (III.6) as

$$
\phi^{B}(z) \phi^{B}(w) \sim \frac{z-w}{z+w},
$$

even though strictly speaking $\frac{z-w}{z+w}=\frac{-2 w}{z+w}+1$ contains also a portion of the normal ordered product (the nonsingular part). 
Lemma III.9 The following formula for the vacuum expectation values on the fermionic side $F_{B}$ holds:

$$
\left\langle 0\left|\phi^{B}\left(z_{1}\right) \phi^{B}\left(z_{2}\right) \ldots \phi^{B}\left(z_{2 n}\right)\right| 0\right\rangle=i_{z} \operatorname{Pf}\left(\frac{z_{i}-z_{j}}{z_{i}+z_{j}}\right)_{i=1}^{2 n} .
$$

Here $i_{z}$ stands for the expansion $i_{z_{1}, z_{1}, \ldots, z_{2 n}}$, and Pf denotes the Pfaffian of an antisymmetric matrix.

This Pfaffian identity is well known, it was first derived in DJKM82]; we will show if follows directly from the bicharacter construction in section $\mathrm{VC}$.

The boson-fermion correspondence of type B is determined once we write the image of the generating fields $\phi^{B}(z)$ (and thus of $T_{-1} \phi^{B}(z)=\phi(-z)$ ) under the correspondence. In order to do that, an essential ingredient is the so-called (see below) twisted Heisenberg field $h(z)$.

Lemma III.10 ([DJKM82], [You89], [Anq13]) Let

$$
h(z):=\frac{1}{4}\left(: \phi^{B}(z) T_{-1} \phi^{B}(z):-1\right)=\frac{1}{4}\left(: \phi^{B}(z) \phi^{B}(-z):-1\right) .
$$

The field $h(z)$ has only odd-indexed modes, $h(z)=\sum_{n \in \mathbb{Z}} h_{2 n+1} z^{-2 n-1}$, and OPE:

$$
h(z) h(w) \sim \frac{z w\left(z^{2}+w^{2}\right)}{2\left(z^{2}-w^{2}\right)^{2}} .
$$

Its modes, $h_{n}, n \in 2 \mathbb{Z}+1$, generate a twisted Heisenberg algebra $\mathcal{H}_{\mathbb{Z}+1 / 2}$ with relations $\left[h_{m}, h_{n}\right]=\frac{m}{2} \delta_{m+n, 0} 1$, $m, n$-odd integers; thus we call the field $h(z)$ "twisted Heisenberg field".

$\mathcal{H}_{\mathbb{Z}+1 / 2}$ has (up-to isomorphism) only one irreducible highest weight module $B_{1 / 2} \cong \mathbb{C}\left[x_{1}, x_{3}, \ldots, x_{2 n+1}, \ldots\right]$.

Lemma III.11 ([DJKM89], YYou89], [Anq13]) The space of states $F_{B}$ can be decomposed as

$$
F_{B}=B_{1 / 2} \oplus B_{1 / 2} \cong \mathbb{C}\left[\mathbb{Z}_{2}\right] \otimes \mathbb{C}\left[x_{1}, x_{3}, \ldots, x_{2 n+1}, \ldots\right]
$$

where the isomorphism is as twisted Heisenberg modules for $\mathcal{H}_{\mathbb{Z}+1 / 2}$. We identify the group algebra $\mathbb{C}\left[\mathbb{Z}_{2}\right]$ with the polynomial algebra $\mathbb{C}\left[e_{B}^{\alpha}, e_{B}^{-\alpha}\right]$ with the extra relation $e_{B}^{2 \alpha} \equiv 1$. Denote $B_{B}:=\mathbb{C}\left[e_{B}^{\alpha}, e_{B}^{-\alpha}\right] \otimes$ $\mathbb{C}\left[x_{1}, x_{3}, \ldots, x_{2 n+1}, \ldots\right]$. In the isomorphism above the image of the vacuum vector $|0\rangle$ (a highest weight vector in $\left.F_{B}\right)$ is $1 \in B_{B}$. The image of the second highest weight vector $\phi_{0}^{B}|0\rangle \in F_{B}$ is $e_{B}^{\alpha} \in B_{B}$.

The (rather peculiar) group algebra notation above, as we will show and use later, is due to the Hopf algebra structure on $B_{B}$, which differs from the super Hopf algebra structure on $F_{B}$. Moreover, we will show that there is a twisted vertex algebra structure, denoted by $T V A\left(B_{B}\right)$, such that $B_{B}$ is the space of states of the bosonic side of the boson-fermion correspondence of type B.

The image of the generating field $\phi^{B}(z)$ (acting on $F_{B}$ ) is the field $e_{B}^{\alpha}(z)$ (acting on $B_{B}$ ), which will determine the correspondence of type B:

Lemma III.12 ([Ang1]]) The bosonization of type B is determined by

$$
\phi^{B}(z) \mapsto e_{B}^{\alpha}(z)=\exp \left(\sum_{k \geq 0} \frac{h_{-2 k-1}}{k+1 / 2} z^{2 k+1}\right) \exp \left(-\sum_{k \geq 0} \frac{h_{2 k+1}}{k+1 / 2} z^{-2 k-1}\right) e^{\alpha} .
$$

The fields $e_{B}^{\alpha}(z)$ and $e_{B}^{\alpha}(-z)=e_{B}^{-\alpha}(z)$ (observe the symmetry) will generate a resulting twisted vertex algebra, which we denote by $T V A\left(B_{B}\right)$.

Lemma III.13 The following formula for the vacuum expectation values on the bosonic side $B_{B}$ holds:

$$
\left\langle 0\left|e_{B}^{\alpha}\left(z_{1}\right) \ldots e_{B}^{\alpha}\left(z_{2 n}\right)\right| 0\right\rangle=i_{z} \prod_{i<j}^{2 n} \frac{z_{i}-z_{j}}{z_{i}+z_{j}} .
$$

Here $i_{z}$ stands for the expansion $i_{z_{1}, z_{1}, \ldots, z_{2 n}}$, and Pf denotes the Pfaffian of an antisymmetric matrix. 
Note that one Heisenberg $\mathcal{H}_{\mathbb{Z}+1 / 2}$-module $B_{1 / 2}$ on its own can be realized as a twisted module for an ordinary super vertex algebra (see [FLM88], BK04] for details), but the point is that we need two of them glued together for the bosonic side of the correspondence. The two of them glued together as above no longer constitute a twisted module for an ordinary super vertex algebra.

Theorem III.14 ([Anq1]]) The boson-fermion correspondence of type $B$ is an isomorphism between the fermionic twisted vertex algebra $T V A\left(F_{B}\right)$ and the bosonic twisted vertex algebra $T V A\left(B_{B}\right)$.

Corollary III.15 The Schur Pfaffian identity follows from the equality between the vacuum expectation values:

$$
A C\left\langle 0\left|\phi^{B}\left(z_{1}\right) \ldots \phi^{B}\left(z_{2 n}\right)\right| 0\right\rangle=\operatorname{Pf}\left(\frac{z_{i}-z_{j}}{z_{i}+z_{j}}\right)_{i, j=1}^{2 n}=\prod_{i<j}^{2 n} \frac{z_{i}-z_{j}}{z_{i}+z_{j}}=A C\left\langle 0\left|e_{B}^{\alpha}\left(z_{1}\right) \ldots e_{B}^{\alpha}\left(z_{2 n}\right)\right| 0\right\rangle .
$$

AC stands for Analytic Continuation.

\section{Examples of twisted vertex algebras: the boson-fermion correspondence of type D-A}

Next are the two examples of a twisted vertex algebra of order 2 which give the two sides of the boson-fermion correspondence of type D-A. The boson-fermion correspondence of type D-A is new, and the bosonisation of type $\mathrm{D}$ is one of the main results of this paper. This correspondence was discussed during the work on both this paper and on [RT12], where the "multilocal fermionization" is discussed. The author thanks K. Rehren for the helpful discussions and a physics point of view on the subject. Here we will show the bosonization of type D (including the split of the neutral fermion space into a direct sum of bosonic spaces) and prove that the boson-fermion correspondence of type D-A is an isomorphism of twisted vertex algebras. The correspondence of type D-A can be generalized to arbitrary order $N \in \mathbb{N}$ (see below, and also [RT12]), but we start with the case of twisted vertex algebras of order two. Again we write $T_{-1}$ instead of $T_{\epsilon}$. The proofs of all the statements and assertions in this section are given in sections $\mathrm{VD}$ and $\mathrm{VK}$

The fermionic side is generated by a single field $\phi^{D}(z)=\sum_{n \in \mathbf{Z}+\mathbf{1} / \mathbf{2}} \phi_{n}^{D} z^{-n-1 / 2}$ with OPE given by:

$$
\phi^{D}(z) \phi^{D}(w) \sim \frac{1}{z-w}, \quad \text { in modes: }\left[\phi_{m}^{D}, \phi_{n}^{D}\right]_{\dagger}=\delta_{m,-n} 1 .
$$

The modes generate a Clifford algebra $C l_{D}$, with underlying space of states denoted by $F_{D}$ - the highest weight representation of $C l_{D}$ with the vacuum vector $|0\rangle$, such that $\phi_{n}|0\rangle=0$ for $n<0$. Here it is recognized that on its own the field $\phi^{D}(z)$ and its descendants $D^{(n)} \phi^{D}(z)$ generate an (ordinary) super-vertex algebra (see e.g., Kac98], KW94]). It is important to note that on its own this super-vertex algebra, called free neutral fermion vertex algebra, cannot be bosonized. But, if we take not only the field $\phi^{D}(z)$, but also its twisted descendant $T_{-1} \phi^{D}(z)=\phi^{D}(-z)$, they together with all their descendants will generate a twisted vertex algebra, denoted by $T V A\left(F_{D}\right)$ which will be bosonized. We call this twisted vertex algebra "free neutral fermion of type D-A". The space of fields of the twisted vertex algebra $T V A\left(F_{D}\right)$ strictly contains the super-vertex algebra $F_{D}$ as a subset, and we can think of it as an orbifolded double cover of the super-vertex algebra.

The boson-fermion correspondence of type D-A is determined once we write the images of the generating fields $\phi^{D}(z)$ and $T_{-1} \phi^{D}(z)=\phi(-z)$ under the correspondence. In order to do that, an essential ingredient is once again the Heisenberg field.

Proposition III.16 Let

$$
h^{D}(z):=\frac{1}{2}: \phi^{D}(z) T_{-1} \phi^{D}(z):=\frac{1}{2}: \phi^{D}(z) \phi^{D}(-z):
$$

The field $h^{D}(z)$ has only odd-indexed modes, $h^{D}(z)=\sum_{n \in \mathbb{Z}} h_{n}^{D} z^{-2 n-1}$ (note the indexing), and OPE:

$$
h^{D}(z) h^{D}(w) \sim \frac{z w}{\left(z^{2}-w^{2}\right)^{2}} .
$$

Its modes, $h_{n}^{D}, n \in \mathbb{Z}$, generate an untwisted Heisenberg algebra $\mathcal{H}_{\mathbb{Z}}$ with relations $\left[h_{m}^{D}, h_{n}^{D}\right]=m \delta_{m+n, 0} 1$, $m, n$-integers; hence we call $h^{D}(z)$ a "Heisenberg field". 
Unlike the twisted Heisenberg algebra, the untwisted Heisenberg algebra has infinitely many irreducible highest weight modules, labeled by the action of $h_{0}^{D}$.

Proposition III.17 The space of states $F_{D}$ can be decomposed as

$$
W=F_{D} \cong \oplus_{i \in \mathbb{Z}} B_{i} \cong \mathbb{C}\left[e^{\alpha}, e^{-\alpha}\right] \otimes \mathbb{C}\left[x_{1}, x_{2}, \ldots, x_{n}, \ldots\right],
$$

$\mathbb{C}\left[e^{\alpha}, e^{-\alpha}\right]$ denotes the Laurent polynomials with one variable $e^{\alpha}$. The isomorphism above is as Heisenberg modules, where $e^{n \alpha}$ denotes the highest weight vector for the irreducible Heisenberg module $B_{n}$, with highest weight $n$.

We denote $B_{D}:=\mathbb{C}\left[e^{\alpha}, e^{-\alpha}\right] \otimes \mathbb{C}\left[x_{1}, x_{2}, \ldots, x_{n}, \ldots\right]$. We will show and use later that $B_{D}$ has a Hopf algebra structure that differs from the super Hopf algebra structure on $F_{D}$. Moreover, we will show that there is a twisted vertex algebra structure, denoted by $T V A\left(B_{D}\right)$, such that $B_{D}$ is the space of states of the bosonic side of the boson-fermion correspondence of type D-A.

Proposition III.18 The boson-fermion correspondence of type D-A is determined by the images of the generating fields $\phi^{D}(z)$ and $T_{-1} \phi^{D}(z)=\phi(-z)$ (which act on $F_{D}$ ), as follows:

$$
\phi^{D}(z) \mapsto e_{\phi}^{-\alpha}(z)+e_{\phi}^{\alpha}(z), \quad(T \phi)^{D}(z)=\phi(-z) \mapsto e_{\phi}^{-\alpha}(z)-e_{\phi}^{\alpha}(z),
$$

where $e_{\phi}^{-\alpha}(z)$ and $e_{\phi}^{\alpha}(z)$ are fields on $B_{D}$ defined by the the formulas

$$
\begin{aligned}
e_{\phi}^{-\alpha}(z) & =\exp \left(-\sum_{n \geq 1} \frac{h_{-n}}{n} z^{2 n}\right) \exp \left(\sum_{n \geq 1} \frac{h_{n}}{n} z^{-2 n}\right) e^{-\alpha} z^{-2 \partial_{\alpha}}=e_{A}^{-\alpha}\left(z^{2}\right), \\
e_{\phi}^{\alpha}(z) & =\exp \left(\sum_{n \geq 1} \frac{h_{-n}}{n} z^{2 n}\right) \exp \left(-\sum_{n \geq 1} \frac{h_{n}}{n} z^{-2 n}\right) e^{\alpha} z^{2 \partial_{\alpha}+1}=z e_{A}^{\alpha}\left(z^{2}\right),
\end{aligned}
$$

Theorem III.19 The boson-fermion correspondence of type $D$ - $A$ is the isomorphism between the fermionic twisted vertex algebra $T V A\left(F_{D}\right)$ and the bosonic twisted vertex algebra $T V A\left(B_{D}\right)$.

Remark III.20 The name "boson-fermion correspondence of type D-A" is given since the fermionic side is a double cover of the well know neutral fermion super vertex algebra that gives the basic representation of $d_{\infty}$, see e.g. KWY98, KW94. On the other hand the operators $e_{A}^{\alpha}(z)$ and $e_{A}^{-\alpha}(z)$ in the right-hand side above are the vertex operators describing the boson-fermion correspondence of type A (II.22), (II.23), as in e.g. Kac98]).

Corollary III.21 The following Pfaffian identity follows from the equality between the vacuum expectation values:

$$
\begin{aligned}
A C\left\langle 0\left|\phi^{D}\left(z_{1}\right) \ldots \phi^{D}\left(z_{2 n}\right)\right| 0\right\rangle=\operatorname{Pf}\left(\frac{1}{z_{i}-z_{j}}\right)_{i, j=1}^{2 n}= & \frac{\sum_{i_{1}<i_{2} \cdots<i_{n}}^{2 n} z_{i_{1}} z_{i_{2}} \cdots z_{i_{n}} \prod_{k<l}^{n}\left(z_{i_{k}}^{2}-z_{i_{l}}^{2}\right) \prod_{p<q}^{n}\left(z_{j_{p}}^{2}-z_{j_{q}}^{2}\right)}{\prod_{k, p}^{n}\left(z_{i_{k}}^{2}-z_{j_{p}}^{2}\right)} \\
& =A C\left\langle 0\left|\left(e_{\phi}^{-\alpha}\left(z_{1}\right)+e_{\phi}^{\alpha}\left(z_{1}\right)\right) \ldots\left(e_{\phi}^{-\alpha}\left(z_{2 n}\right)+e_{\phi}^{\alpha}\left(z_{2 n}\right)\right)\right| 0\right\rangle
\end{aligned}
$$

$A C$ stands for Analytic Continuation, and $\left\{j_{1}, j_{2}, \ldots j_{n}\right\}=\{1,2, \ldots 2 n\} \backslash\left\{i_{1}, i_{2}, \ldots i_{n}\right\}$.

\section{Examples of twisted vertex algebras: the boson-fermion correspondence of type D-A of order $N$}

The boson-fermion correspondence of type D-A from the previous section can be generalized to general order $N$ as follows. We again consider the free field $\phi^{D}(z)=\sum_{n \in \mathbf{Z}+\mathbf{1} / \mathbf{2}} \phi_{n}^{D} z^{-n-1 / 2}$, with OPEs with itself given by (III.11). Let $\epsilon$ be an $N$-th order primitive root of unity and consider the following twisted vertex algebra descendants $T_{\epsilon}^{i} \phi^{D}(z)=\phi^{D}\left(\epsilon^{i} z\right)$, for any $0 \leq i \leq N-1$, with OPEs

$$
T_{\epsilon}^{i} \phi^{D}(z) T_{\epsilon}^{j} \phi^{D}(w) \sim \frac{1}{\epsilon^{i} z-\epsilon^{j} w} .
$$

Such OPEs are not allowed in a super vertex algebra, but are allowed in a twisted vertex algebra. These mean that on its own each of the fields $T_{\epsilon}^{i} \phi^{D}(z)$, for any $0 \leq i \leq N-1$ will generate a super vertex algebra, but the $N$ of them "glued" together form a twisted vertex algebra. Again, this resembles the gluing together of the $N$ sheets of the $N$-th root Riemann surface. 
Lemma III.22 The field $h(z)$ given by

$$
h(z)=\frac{1}{N} \sum_{i=0}^{N-1} \epsilon^{i-1}: T_{\epsilon}^{i-1} \phi^{D}(z) T_{\epsilon}^{i} \phi^{D}(z):=\sum_{n \in \mathbb{Z}} h_{n} z^{-N n-1}
$$

is a Heisenberg field with OPE

$$
h(z) h(w) \sim \frac{z^{N-1} w^{N-1}}{\left(z^{N}-w^{N}\right)^{2}} .
$$

Thus the commutation relations $\left[h_{m}, h_{n}\right]=m \delta_{m+n, 0} 1$ for the Heisenberg algebra $\mathcal{H}_{\mathbb{Z}}$ hold.

Lemma III.23 Let

$$
e_{\phi}^{\alpha}(w)=\frac{1}{N}\left(\sum_{i=0}^{N-1} \epsilon^{-i} T^{i} \phi^{D}(w)\right), \quad e_{\phi}^{\epsilon^{k} \alpha}(w)=\frac{1}{N}\left(\sum_{i=0}^{N-1} \epsilon^{(k-1) i} T^{i} \phi^{D}(w)\right) .
$$

The boson-fermion correspondence of order $N$ is given by

$$
e_{\phi}^{\epsilon^{k} \alpha}(z) \mapsto \exp \left(\epsilon^{-k} \sum_{n \geq 1} \frac{h_{-n}}{n} z^{N n}\right) \exp \left(\epsilon^{k} \sum_{n \geq 1} \frac{h_{n}}{n} z^{-N n}\right) e_{\phi}^{\epsilon^{k} \alpha} z^{1-k+N \partial_{\alpha}},
$$

where $e_{\phi}^{\epsilon^{k} \alpha}$ is identified as the highest weight vectors of the Heisenberg submodule.

\section{BICHARACTER CONSTRUCTION: CONSTRUCTING EXAMPLES OF TWISTED VERTEX ALGEBRAS}

\section{A. Super bicharacters and free Leibnitz modules}

In this section we recall the components of the super-bicharacter construction (the super case was introduced in [Ang08], generalizing [Bor01]).

Notation IV.1 Henceforth we will assume that $M$ is a supercommutative and supecocommutative bialgebra. To unclutter the language, we will just write commutative, cocommutative, omitting the term "super" as long as the parity is clear from the context.

Definition IV.2 (Super-bicharacter) Define a bicharacter $r$ on $M$ to be a linear map $r: M \otimes M \rightarrow$ $\mathbf{F}^{N}(z, w)$, such that

$$
\begin{aligned}
r_{z_{1}, z_{2}}(1 \otimes a) & =\eta(a)=r_{z, w}(a \otimes 1), \\
r_{z, w}(a b \otimes c) & =\sum(-1)^{\tilde{b} c^{\prime}} r_{z, w}\left(a \otimes c^{\prime}\right) r_{z, w}\left(b \otimes c^{\prime \prime}\right), \\
r_{z, w}(a \otimes b c) & =\sum(-1)^{\tilde{a^{\prime \prime}} \tilde{b}} r_{z, w}\left(a^{\prime} \otimes b\right) r_{z, w}\left(a^{\prime \prime} \otimes c\right) .
\end{aligned}
$$

We say that a bicharacter $r$ is even if $r_{z, w}(a \otimes b)=0$ whenever $\tilde{a} \neq \tilde{b}$.

From now on we will always work with even bicharacters. In most cases there are no nontrivial bicharacters which are not even (see $[$ Ang08]). The identity bicharacter is given by $r(a \otimes b)=\eta(a) \otimes \eta(b)$.

Remark IV.3 The notion of super bicharacter is similar to the notion of a twist induced by Laplace pairing (or the more general concept of a Drinfeld twist) as described in [BFFO04].

Definition IV.4 (Symmetric bicharacter) The transpose bicharacter $r^{\tau}$ of a bicharacter $r$ is defined by

$$
r_{z, w}^{\tau}(a \otimes b)=r_{w, z} \circ \tau(a \otimes b) .
$$

A bicharacter $r$ is called symmetric if $r=r^{\tau}$. 
Definition IV.5 $\left(\mathbf{H}_{\mathbf{T}_{\epsilon}}^{\mathbf{N}} \otimes \mathbf{H}_{\mathbf{T}_{\epsilon}}^{\mathbf{N}}\right.$-covariant bicharacter) Let $M$ be a Hopf supercommutative and supecocommutative superalgebra, $r$ be a bicharacter on $M$. Suppose in addition $M$ is an $H_{T_{\epsilon}}^{N}$-module algebra. We say that the bicharacter $r$ is $H_{T_{\epsilon}}^{N} \otimes H_{T_{\epsilon}}^{N}$-covariant if it additionally satisfies :

$$
r_{z, w}(h(a) \otimes g(b))=h_{z} g_{w} \cdot r_{z, w}(a \otimes b),
$$

for all $a, b \in M, h, g \in H_{T_{\epsilon}}^{N}$.

We recall the following result from [Bor01], generalized to the super case:

Lemma/Definition IV.6 (Free H-Leibnitz module) Suppose $M$ is a super commutative algebra and $H$ is an entirely even cocommutative bialgebra. Then there is a universal supercommutative algebra $H(M)$ such that there is a map $h \otimes m \rightarrow h m:=h(m)$ from $H \otimes M$ to $H(M)$ such that $H(M)$ is a left module for $H$ and

$$
h(m n)=\sum h^{\prime}(m) h^{\prime \prime}(n), \quad h(1)=\eta(h),
$$

for any $m, n \in M, h \in H$. We will call $H(M)$, defined as above, the "free $H$ Leibnitz module of $M$ " (or universal $H$-Leibnitz module of $M$ ).

An $H$-module with the properties (IV.6) is by definition an $H$-module algebra (see e.g. Kas95]), thus $H(M)$ is an $H$-module algebra. It is the universal $H$-module algebra containing $M$ in the super-commutative category.

Remark IV.7 If $M$ is supercommutative and supercocommutative bialgebra (or Hopf algebra), then so is $H(M)$. The extension of comultiplication and the counit from $M$ to $H(M)$ is as follows: If $a \in M, h \in H$ we have $h a \in H(M)$ and we define

$$
\triangle(h a)=\sum h^{\prime} a^{\prime} \otimes h^{\prime \prime} a^{\prime \prime}, \quad \eta(h a)=\eta(h) \eta(a) .
$$

It is easy to check that the comultiplication and the counit defined as above will turn $H(M)$ into a bialgebra. A source of examples of $H_{T_{\epsilon}}^{N} \otimes H_{T_{\epsilon}}^{N}$-covariant bicharacters is as follows:

Lemma IV.8 ([Bor01]) (Extension of bicharacters) Let $r: M \otimes M \rightarrow \mathbf{F}^{N}(z, w)$ be a bicharacter on $M$. Then there exist an $H_{T_{\epsilon}}^{N} \otimes H_{T_{\epsilon}}^{N}$-covariant bicharacter on the free Leibnitz module $H_{T_{\epsilon}}^{N}(M)$, such that its restriction to $M$ is the bicharacter $r$.

We will denote the "induced" bicharacter also by $r$.

Proof: To define the bicharacter on $H_{T_{\epsilon}}^{N}(M)$ we note that all elements in $H_{T_{\epsilon}}^{N}(M)$ are generated, as an algebra, from elements of the form $a=h \bar{a}, b=g \bar{b}$ for some $\bar{a}, \bar{b} \in M, g, h \in H$. Thus define a bicharacter

$$
r: H_{T_{\epsilon}}^{N}(M) \otimes H_{T_{\epsilon}}^{N}(M) \rightarrow \mathbf{F}^{N}(z, w) \quad \text { by } \quad r_{z, w}(a \otimes b)=h_{z} g_{w} \cdot r_{z, w}(\bar{a} \otimes \bar{b})
$$

and extend it by linearity and using multiplicativity ( (IV.2) and (IV.31) $)$ of the bicharacter to the whole of $H_{T_{\epsilon}}^{N}(M)$. The extended bicharacter $r$ on $H_{T_{\epsilon}}^{N}(M)$ is by construction $H_{T_{\epsilon}}^{N} \otimes H_{T_{\epsilon}}^{N}$-covariant.

\section{B. Examples of free Leibnitz modules}

Most of the vector spaces underlying our vertex algebras in this paper are going to be free Leibnitz modules. The first two types of examples of free Leibnitz modules are entirely even, or bosonic.

Example IV.9 (The free Leibnitz modules $H_{D}(\mathbb{C}[h])$ and $H_{T_{\epsilon}}^{N}(\mathbb{C}[h])$ )

The free $H_{D}$-Leibnitz module over the algebra $\mathbb{C}[h]$ (the polynomial algebra of a single variable, considered as a Hopf algebra with $h$ a primitive element) is isomorphic to the polynomial algebra $\mathbb{C}\left[x_{1}, x_{2}, \ldots, x_{n}, \ldots\right]$. We can identify $x_{1}=h$ and $D^{(n)} h=D^{(n)} x_{1}=x_{n+1}$. From equation (II.3), we have that $x_{n}=D^{(n-1)} h$ are primitive: according to (IV.7)

$$
\triangle\left(D^{(l)} h\right)=\left(\sum_{p+q=l} D^{(p)} \otimes D^{(q)}\right)(h \otimes 1+1 \otimes h)=D^{(l)} h \otimes 1+1 \otimes D^{(l)} h,
$$


one similarly checks the counit and the anitpode. These variables commute and generate $H_{D}(\mathbb{C}[h])$. Thus $\mathbb{C}\left[x_{1}, x_{2}, \ldots, x_{n}, \ldots\right]$ is isomorphic to the free $H_{D}$ module-algebra over $\mathbf{C}[h]$.

The free Leibnitz module $H_{T_{\epsilon}}^{N}(\mathbb{C}[h])$ is isomorphic as a Hopf algebra to the polynomial algebra with $k$ groups of variables: $\mathbb{C}\left[x_{1}^{0}, x_{2}^{0}, \ldots, x_{n}^{0}, \ldots, x_{1}^{N-1}, x_{2}^{N-1}, \ldots, x_{n}^{N-1}, \ldots,\right]$, by identifying

$$
x_{l}^{k}=T^{k} D^{(l)} h, \quad k=0, \ldots, N-1, \quad n=0,1, \ldots, l, \ldots
$$

\section{Example IV.10 (The free Leibnitz modules over free abelian group algebras)}

Let $L_{1}=\mathbb{C}[\mathbb{Z} \alpha]$ be the group algebra of the rank-one free abelian group $\mathbb{Z} \alpha$. The group algebra is generated by $e^{m \alpha}, m \in \mathbb{Z}$, with relations $e^{m \alpha} e^{n \alpha}=e^{(m+n) \alpha}, e^{0}=1$. Note that as an algebra $L_{1}=\mathbb{C}\left[e^{\alpha}, e^{-\alpha}\right]$, with the relation $R: e^{\alpha} e^{-\alpha}=1$. The Hopf algebra structure is determined by $e^{\alpha}$ and $e^{-\alpha}$ being grouplike.

Lemma IV.11 The free $H_{D}$ Leibnitz module $H_{D}\left(L_{1}\right)$ is isomorphic as an algebra to $L_{1} \otimes \mathbb{C}[h]$.

Proof: Since $H_{D}\left(L_{1}\right)$ is a free Leibnitz module, we can define an element $h=\left(D e^{\alpha}\right) e^{-\alpha}$. It follows that $h$ is primitive, we have

$$
\Delta(h)=\Delta\left(D e^{\alpha}\right) \Delta\left(e^{-\alpha}\right)=\left(D e^{\alpha} \otimes e^{\alpha}+\left(e^{\alpha} \otimes D e^{\alpha}\right)\right)\left(e^{-\alpha} \otimes e^{-\alpha}\right)=h \otimes 1+1 \otimes h .
$$

Similarly one checks that $\epsilon(h)=0$. Thus, since $H_{D}\left(L_{1}\right)$ is a free Leibnitz module, it has the subalgebra $H_{D}(\mathbf{C}[h])=\mathbf{C}\left[x_{1}, x_{2}, \ldots, x_{n}, \ldots\right]$. Thus, $L_{1} \otimes H_{D}(\mathbf{C}[h])$ is (isomorphic to) a subalgebra in $H_{D}\left(L_{1}\right)$. Conversely, for any $m \in \mathbf{Z}$, since $D e^{\alpha}=h \cdot e^{\alpha}$ we can write

$$
D e^{m \alpha}=D\left(e^{\alpha}\right)^{m}=m\left(D e^{\alpha}\right)\left(e^{\alpha}\right)^{m-1}=m\left(h e^{\alpha}\right)\left(e^{\alpha}\right)^{m-1}=m h \cdot e^{m \alpha}
$$

thus for any $m, n \in \mathbf{Z}$ the element $D^{n}\left(e^{m \alpha}\right)$ is in $M \otimes H_{D}(\mathbf{C}[h])$, and so $H_{D}\left(L_{1}\right)$ is (isomorphic to) a subalgebra in $M \otimes H_{D}(\mathbf{C}[h])$. Thus $H_{D}\left(L_{1}\right)$ is isomorphic to $L_{1} \otimes \mathbb{C}[h] \simeq L_{1} \otimes \mathbf{C}\left[x_{1}, x_{2}, \ldots, x_{n}, \ldots\right], n \in \mathbf{N}$.

Note that the primitive element $h$ is of particular importance for the boson-fermion correspondences, as it generates a Heisenberg subalgebra for a variety of choices for a bicharacter.

The free Leibnitz module $H_{T_{\epsilon}}^{N}\left(L_{1}\right)$ is isomorphic to $L_{N} \otimes H_{T_{\epsilon}}^{N}(\mathbb{C}[h])$, where $L_{N}$ is the group algebra $L_{N}=$ $\mathbb{C}\left[\mathbb{Z} \alpha_{1}, \mathbb{Z} \alpha_{2}, \ldots \mathbb{Z} \alpha_{N}\right]$ of the free abelian group of rank $\mathrm{N}$ (one can identify $T^{k} e^{\alpha}$, which is grouplike, with $e^{\alpha_{k}}$ ).

One proceeds similarly with the free Leibnitz modules over the free abelian group of any rank.

The other examples we will use throughout the paper are fermionic, or super algebras.

Example IV.12 (The free Leibnitz modules $H_{D}(\mathbb{C}\{\phi\})$ and $H_{T_{\epsilon}}^{N}(\mathbb{C}\{\phi\})$ )

Let $\mathbb{C}\{\phi\}$ be the Grassmann algebra generated by one odd primitive element $\phi, \phi \cdot \phi=0$. Then the free Leibnitz module $H_{D}(\mathbb{C}\{\phi\})$ is the Grassmann algebra with odd anticommuting generators $\phi^{n}=D^{(n)} \phi$, $\phi^{n} \phi^{m}+\phi^{m} \phi^{n}=0$, which one checks to be primitive.

Similarly, the free Leibnitz module $H_{T_{\epsilon}}^{N}(\mathbb{C}\{\phi\})$ is the Grassmann algebra with odd anticommuting generators

$$
\phi^{n, k}=D^{(n)} T_{\epsilon}^{k} \phi, \quad k=0, \ldots, N-1, \quad n=0,1, \ldots, l, \ldots
$$

Note that the ordering of the operators $D^{(n)}$ and $T_{\epsilon}^{k}$ matters; one should be careful to be consistent, as it may result in rescaling of the basis: $\phi^{n, k}=D^{(n)} T_{\epsilon}^{k} \phi=\epsilon^{k n} T_{\epsilon}^{k} D^{(n)} \phi$.

Of particular interest for the boson-fermion correspondences is going the case of $N=2$ : the free Leibnitz module $H_{T_{\epsilon}}^{2}(\mathbb{C}\{\phi\})$ is algebra-isomorphic to $H_{D}(\mathbb{C}\{\phi, T \phi\})$, where write $T=T_{\epsilon}=T_{-1}(\epsilon=-1$ in this case). Both the boson-fermion correspondences (the type B, and the type D-A) have $H_{D}(\mathbb{C}\{\phi, T \phi\})$ as underlying space of fields on its fermionic side. Of particular interest is the element

$$
h_{\phi}=\phi T \phi, \quad \text { with } T h_{\phi}=-h_{\phi}
$$

This element is even, and although it is not primitive, we will see in the later sections that the element $h_{\phi}$ generates a Heisenberg subalgebra for particular choices of bicharacter.

The last example of free Leibnitz modules in this paper is the following:

Example IV.13 (The free Leibnitz modules $H_{D}(\mathbb{C}\{\phi, \psi\})$ and $H_{T_{\epsilon}}^{N}(\mathbb{C}\{\phi, \psi\})$ )

Let $\mathbb{C}\{\phi, \psi\}$ be the Grassmann algebra generated by two odd primitive element $\phi, \psi$, such that $\phi \cdot \phi=\psi \cdot \psi=$ $\phi \psi+\psi \phi=0$. Then the free Leibnitz module $H_{D}(\mathbb{C}\{\phi, \psi\})$ is the Grassmann algebra with odd anticommuting primitive generators $\phi^{n}=D^{(n)} \phi, \psi^{n}=D^{(n)} \psi$. The $H_{D}(\mathbb{C}\{\phi, \psi\})$ is the underlying space on the fermionic side of the boson-fermion correspondence of type A. Of particular interest is the element $h_{\phi, \psi}=\phi \psi$, which is again even, but not primitive, and has similar coproduct as the element $h_{\phi}$ above. $h_{\phi, \psi}$ also generates a Heisenberg subalgebra for particular choices of the bicharacter as we will see in the later sections (an example appeared in [Ang08]). Similarly $H_{T_{\epsilon}}^{N}(\mathbb{C}\{\phi, \psi\})$ is isomorphic to $H_{D}\left(\mathbb{C}\left\{\phi, T_{\epsilon} \phi, \ldots, T_{\epsilon}^{N-1} \phi, \psi, T_{\epsilon} \psi, \ldots, T_{\epsilon}^{N-1} \psi\right\}\right)$. 


\section{Exponential map and its properties; Nonsingular twisted vertex algebras}

Definition IV.14 (Nonsingular vertex algebra) We call a twisted vertex algebra nonsingular if the analytic continuations $X_{z, w, 0}(a \otimes b \otimes c)$ have no poles for any $a, b, c \in V$.

For super vertex algebras this definition coincides with the notion of a holomorphic super vertex algebra introduced in the previous literature (see for example [Kac98], LL04]). A holomorphic super-vertex algebra is in fact just a commutative associative unital differential algebra: if $V$ is a holomorphic super-vertex algebra, for any $a, b \in V$ we have (see for example [Kac98], LL04]):

$$
Y(a, z) b=\left(e^{z D} a\right) b, \quad \text { where } \quad e^{z D}=\sum_{n \geq 0} z^{n} D^{(n)} .
$$

Thus the fields in a holomorphic super-vertex algebra are uniquely determined by the unique "exponential map" $e^{z D}$. In the case of twisted vertex algebras the situation is not as simple, as the "exponential map" is not unique. There are a variety of examples of "exponential maps" for twisted vertex algebras that would satisfy the properties of a nonsingular twisted vertex algebra. Note that for twisted vertex algebras, we also have the concept of a projection map, thus we really consider pairs of exponential and projection maps that satisfy the properties of a nonsingular twisted vertex algebra. Hence we will proceed to define a standard pair of projection and exponential maps on given free $H_{T_{\epsilon}}^{N}$ Leibnitz modules. In what follows let $M$ be a commutative cocommutative Hopf algebra, $V$ be the free Leibnitz module $V=H_{T_{\epsilon}}^{N}(M)$. Note that the free Leibnitz module $W=H_{D}(M)$ is a sub-Hopf algebra of $V$, and thus we can use the exponential map $e^{z D}$ on $W$. Moreover, each element in $V$ can be written uniquely as a linear combination of elements of the form $a=\prod_{i=0}^{N-1} a_{i}$, where $a_{i}=T_{\epsilon}^{i} \bar{a}_{i}$, for some $\bar{a}_{i} \in W$.

Definition IV.15 (T-projection Map $\pi_{\mathbf{T}}$ ) Let $M$ be a commutative cocommutative Hopf algebra, let $V$ be the free Leibnitz module $V=H_{T_{\epsilon}}^{N}(M), W=H_{D}(M)$, and let $a \in V$ is such that $a=\prod_{i=0}^{N-1} a_{i}$, where $a_{i}=T_{\epsilon}^{i} \bar{a}_{i}$ for some $\bar{a}_{i} \in W$. Define the projection map $\pi_{T}: V \rightarrow W$ to be the algebra homomorphism map defined by:

$$
\pi_{T}\left(a_{i}\right)=\bar{a}_{i},, \quad i=1, \ldots, N-1, \quad \pi_{T}(a)=\prod_{i=0}^{N-1} \bar{a}_{i} .
$$

Definition IV.16 (Exponential Map $\mathcal{E}_{\mathbf{z}}$ ) Let as above $a_{i}=T_{\epsilon}^{i} \bar{a}_{i}, \bar{a}_{i} \in W, a=\prod_{i=0}^{N-1} a_{i}$. Define the map $\mathcal{E}_{z}: V \rightarrow W[[z]]$ to be the algebra homomorphism map such that

$$
\begin{aligned}
& \mathcal{E}_{z}\left(\bar{a}_{i}\right)=e^{z D} \bar{a}_{i}, \text { for any } \bar{a}_{i} \in W \\
& \mathcal{E}_{z}\left(a_{i}\right)=e^{\epsilon^{i} z D} \bar{a}_{i}, \quad i=0, \ldots, N-1 ; \\
& \mathcal{E}_{z}\left(\prod_{i=0}^{N-1} a_{i}\right)=\prod_{i=0}^{N-1} e^{\epsilon^{i} z D} \bar{a}_{i} ;
\end{aligned}
$$

and extend $\mathcal{E}_{z}$ by linearity to $V$.

Example IV.17 This example points out that one has to be careful with the specific order between $D^{(n)}$ and $T_{\epsilon}^{i}$ which we implicitly used in the definition of the exponential map $\mathcal{E}_{z}$. Let as above $a_{i}=T_{\epsilon}^{i} \bar{a}_{i}, \bar{a}_{i} \in W$, and let $n \in \mathbb{N}$. To calculate $\mathcal{E}_{z}\left(D^{(n)} a_{i}\right)$, we need

$$
D^{(n)} a_{i}=D^{(n)} T_{\epsilon}^{i} \bar{a}_{i}=\epsilon^{n i} T_{\epsilon}^{i} D^{(n)} \bar{a}_{i} ;
$$

where now $D^{(n)} \bar{a}_{i} \in W$, and thus

$$
\mathcal{E}_{z}\left(D^{(n)} a_{i}\right)=\epsilon^{n i} \epsilon^{\epsilon^{i} z D}\left(D^{(n)} \bar{a}_{i}\right)=\epsilon^{n i} D^{(n)}\left(e^{\epsilon^{i} z D} \bar{a}_{i}\right)=\epsilon^{n i} D^{(n)} \mathcal{E}_{z}\left(a_{i}\right) .
$$

This equality plays part in the "transfer of action" property of $\mathcal{E}_{z}$, and is also used in proving the Modified Expansion property of the exponential map. 
Lemma IV.18 (Properties of the Exponential Map $\mathcal{E}_{\mathbf{z}}$ ) Let $M$ be a commutative cocommutative Hopf algebra, let $V$ be the free Leibnitz module $V=H_{T_{\epsilon}}^{N}(M), r$ is a $H_{T_{\epsilon}}^{N} \otimes H_{T_{\epsilon}}^{N}$-covariant bicharacter on $V$. Let $\pi_{T}: V \rightarrow W$ and $\mathcal{E}_{z}: V \rightarrow W[[z]]$ be the pair projection-exponential map defined above. This pair of maps satisfies the following properties:

- Vacuum property: $\mathcal{E}_{z}(1)=1$, where 1 is the unit in $V$;

- Modified creation property: $\left.\mathcal{E}_{z}(a)\right|_{z=0}=\pi_{T}(a)$, for any $a \in V$;

- Transfer of action: $\mathcal{E}_{z}(h a)=h_{z} \mathcal{E}_{z}(a)$, for any $h \in H_{T_{\epsilon}}^{N}, a \in V$;

- Multiplicativity: $\mathcal{E}_{z}(a b)=\mathcal{E}_{z}(a) \mathcal{E}_{z}(b)$, for any $a, b \in V$;

- Grouplike: $\triangle \mathcal{E}_{z}(a)=\mathcal{E}_{z}\left(a^{\prime}\right) \otimes \mathcal{E}_{z}\left(a^{\prime \prime}\right)$;

- Compatibility with bicharacters: $i_{z, w} r_{z, w}(a \otimes b)=r_{z, 0}\left(a \otimes \mathcal{E}_{w}(b)\right)$, for any $a, b \in V$.

- Modified expansion: $\mathcal{E}_{z}(a)=\sum_{n \geq 0}\left(z-\epsilon^{i} w\right)^{n} \mathcal{E}_{w}\left(T^{i} D^{(n)} a\right)$,

Proof: The proofs for most of the properties are straightforward, and use the similar properties of the ordinary exponential map $e^{z D}$ and the definition of the map $\mathcal{E}_{z}$ via the projection map.

Remark IV.19 Note that as we saw in the example IV.17 above $D^{(n)}$ and $\mathcal{E}_{w}$ do not commute. Thus even though $\sum_{n \geq 0}\left(z-\epsilon^{i} w\right)^{n} \mathcal{E}_{w}\left(T^{i} D^{(n)} a\right)=\sum_{n \geq 0} \epsilon^{-i n}\left(z-\epsilon^{i} w\right)^{n} \mathcal{E}_{w}\left(D^{(n)} T a\right)$, and $\sum_{n \geq 0} \epsilon^{-i n}\left(z-\epsilon^{i} w\right)^{n} D^{(n)}=$ $e^{\epsilon^{-i}\left(z-\epsilon^{-i} w\right) D}$, the modified expansion property can not be rewritten using $e^{\epsilon^{-i}\left(z-\epsilon^{-i} w\right) D}$. That is the reason for the lack of a "modified associativity" property.

Lemma IV.20 (Nonsingular twisted vertex algebra) Let $M$ be a commutative cocommutative Hopf algebra, let $V$ be the free Leibnitz module $V=H_{T_{\epsilon}}^{N}(M)$, let $\pi_{T}: V \rightarrow W$ be the projection map from definition IV.15. The map $\mathcal{E}_{z}: V \rightarrow W[[z]]$ defines a structure of a nonsingular twisted vertex algebra $\left(V, W, \pi=\pi_{T}, Y\right)$ by:

$$
Y(a, z) \pi_{T}(b)=\mathcal{E}_{z}(a) \cdot \pi_{T}(b) \quad \text { for any } \quad a, b \in V \text {. }
$$

\section{Vertex operators, analytic continuations, OPEs and normal ordered products from a bicharacter}

In this subsection we combine together the bicharacter construction to produce fields and vertex algebras from a bicharacter. A space of fields in the vertex algebra is given by the free Leibnitz module $V=H_{T_{\epsilon}}^{N}(M)$, a space of states by the free Leibnitz module $W=H_{D}(M) \subset V$. This is part of the data needed for a twisted vertex algebra. Now we will define the fields and the field-state correspondence via a bicharacter.

Definition IV.21 (Two-variable fields from a bicharacter) Let $M$ be a commutative cocommutative Hopf algebra, let $V$ be the free Leibnitz module $V=H_{T_{\epsilon}}^{N}(M), r$ a $H_{T_{\epsilon}}^{N} \otimes H_{T_{\epsilon}}^{N}$-covariant bicharacter on $V$ with values in $\mathbf{F}^{N}(z, w)^{+, w}, W=H_{D}(M)$ be the free $H_{D}$-Leibnitz sub-module-algebra of $V$. Let $\mathcal{E}_{z}$ be the exponential map $\mathcal{E}_{z}: V \rightarrow W[[z]]$ defined in IV.16. Define a singular multiplication map

$$
X_{z, w}: V^{\otimes 2} \rightarrow W[[z, w]] \otimes \mathbf{F}^{N}(z, w),
$$

by

$$
X_{z, w}(a \otimes b)=\sum(-1)^{a^{\tilde{\prime}} \tilde{b}^{\prime}}\left(\mathcal{E}_{z} a^{\prime}\right)\left(\mathcal{E}_{w} b^{\prime}\right) r_{z, w}\left(a^{\prime \prime} \otimes b^{\prime \prime}\right),
$$

where $a, b$ are homogeneous elements of the super space $V$. The map $X_{z, w}$ is extended by linearity to $V$.

Definition IV.22 (Vertex operators $Y(a, z)$ and field-state correspondence) Let $V, W, \mathcal{E}_{z}$ be as above, $\pi_{T}: V \rightarrow W$ be the projection map defined in IV.15. Define the vertex operator $Y(a, z)$ associated to a homogeneous element $a \in V$, by

$$
Y(a, z) \pi_{T}(b)=X_{z, 0}(a \otimes b)=\sum(-1)^{\tilde{a}^{\prime \prime} \tilde{b}^{\prime}}\left(\mathcal{E}_{z} a^{\prime}\right) \pi_{T}\left(b^{\prime}\right) r_{z, 0}\left(a^{\prime \prime} \otimes b^{\prime \prime}\right),
$$

for any homogeneous element $b \in V$. Extend by linearity to any elements $a, b \in V$. Then $Y(a, z)$ is a field on $W$ and the map $Y: a \in V \rightarrow Y(a, z)$ is a field-state correspondence for the twisted vertex algebra with space of fields $V$ and space of states $W$. 
Here we are implicitly using the modified creation property of the exponential map (lemma IV.18).

Remark IV.23 This definition is consistent, i.e., for each $\bar{b} \in W$ the vertex operator acting on $\bar{b}$ is independent from the choice of the $b \in V$ used in the definition, due to the following: If $\bar{b}=\pi_{T}\left(b_{1}\right)=\pi_{T}\left(b_{2}\right)$, then from the $H_{T_{\epsilon}}^{N} \otimes H_{T_{\epsilon}}^{N}$-covariance of the bicharacter $r$ it follows that $r_{z, 0}\left(a \otimes b_{1}\right)=r_{z, 0}\left(a \otimes b_{2}\right)=r_{z, 0}(a \otimes \bar{b})$. Also, since the map $\pi_{T}(b)$ is a surjection, this definition is sufficient for any $\bar{b} \in W$.

Remark IV.24 Note that any $H_{T_{\epsilon}}^{N} \otimes H_{T_{\epsilon}}^{N}$-covariant bicharacter $r$ will produce a (different) field-state correspondence, thus even with the same space of fields $V$ and space of states $W$ we can get a variety of examples of field-state correspondences by choosing different bicharacters on $V$.

Lemma IV.25 ( $\mathbf{n}=\mathbf{2}$ Analytic continuation) Let $V, W, \mathcal{E}_{z}, \pi_{T}: V \rightarrow W$ be as above. We have for any $a, b \in V$

$$
\begin{aligned}
& Y(a, z) 1=\mathcal{E}_{z} a \\
& i_{z, w} X_{z, w}(a \otimes b)=Y(a, z) \mathcal{E}_{w} b=Y(a, z) Y(b, w) 1 .
\end{aligned}
$$

Proof: (IV.23) follows from the vacuum property of the exponential map (see lemma IV.18). Note that since $W=H_{D}(M)$ is a Hopf subalgebra of $V$, the unit 1 is in $W$. Thus we have for $a \in V$ a homogeneous element

$$
Y(a, z) 1=\sum(-1)^{0}\left(\mathcal{E}_{z} a^{\prime}\right) 1 r_{z, 0}\left(a^{\prime \prime} \otimes 1\right)=\sum\left(\mathcal{E}_{z} a^{\prime}\right) \eta\left(a^{\prime \prime}\right)=\mathcal{E}_{z} a .
$$

The equation (IV.24) follows from the "compatibility with bicharacters" and the "grouplike" properties of the exponential map (see lemma IV.18):

$$
\begin{aligned}
i_{z, w} X_{z, w}(a \otimes b) & =\sum(-1)^{\tilde{a^{\prime \prime}} \tilde{b}^{\prime}}\left(\mathcal{E}_{z} a^{\prime}\right)\left(\mathcal{E}_{w} b^{\prime}\right) i_{z, w} r_{z, w}\left(a^{\prime \prime} \otimes b^{\prime \prime}\right)=\sum(-1)^{\tilde{a}^{\prime \prime} \tilde{b}^{\prime}}\left(\mathcal{E}_{z} a^{\prime}\right)\left(\mathcal{E}_{w} b^{\prime}\right) r_{z, 0}\left(a^{\prime \prime} \otimes \mathcal{E}_{w} b^{\prime \prime}\right) \\
& =\sum(-1)^{\tilde{a^{\prime \prime}} \tilde{b}^{\prime}}\left(\mathcal{E}_{z} a^{\prime}\right)\left(\mathcal{E}_{w} b\right)^{\prime} r_{z, 0}\left(a^{\prime \prime} \otimes\left(\mathcal{E}_{w} b\right)^{\prime \prime}\right)=Y(a, z) \mathcal{E}_{w} b,
\end{aligned}
$$

since $\pi_{T}\left(\mathcal{E}_{w} b\right)=\mathcal{E}_{w} b$ for any $b \in V$.

Thus we have established a field-state correspondence $Y$, and in fact have also proved that it satisfies the analytic continuation property of twisted vertex algebras for $n=2$. It is immediate that this field-state correspondence satisfies also the following required properties for a twisted vertex algebra:

Lemma IV.26 Let $V, W, \mathcal{E}_{z}, \pi=\pi_{T}: V \rightarrow W, Y: a \in V \rightarrow Y(a, z)$ be as above. This data satisfies the following properties:

- vacuum axiom: $Y(1, z)=I d_{W}$;

- modified creation axiom: $\left.Y(a, z)|0\rangle\right|_{z=0}=\pi(a)$, for any $a \in V$;

- transfer of action: $Y(h a, z)=h_{z} \cdot Y(a, z)$ for any $h \in H_{T_{\epsilon}}^{N}$.

Proof: The vacuum property and the modified creation property follow immediately from the corresponding properties of the exponential map. For the transfer of action property:

$$
Y(h a, z) \pi_{T}(b)=X_{z, 0}(h a \otimes b)=\sum(-1)^{\tilde{a^{\prime \prime}} \tilde{b}^{\prime}}\left(\mathcal{E}_{z}(h a)^{\prime}\right) \pi_{T}\left(b^{\prime}\right) r_{z, 0}\left((h a)^{\prime \prime} \otimes b^{\prime \prime}\right),
$$

which from the property of free Leibnitz modules equals

$$
\sum(-1)^{\tilde{a}^{\prime \prime}} \tilde{b}^{\prime}\left(\mathcal{E}_{z}\left(h^{\prime} a^{\prime}\right) \pi_{T}\left(b^{\prime}\right) r_{z, 0}\left(h^{\prime \prime} a^{\prime \prime} \otimes b^{\prime \prime}\right)\right.
$$

From the transfer of action property of the exponential map, and the covariance of the bicharacter this equals

$$
\begin{aligned}
& \sum(-1)^{a^{\prime \prime} \tilde{b}^{\prime}}\left(\left(h^{\prime}\right)_{z} \cdot \mathcal{E}_{z}\left(a^{\prime}\right)\right) \pi_{T}\left(b^{\prime}\right)\left(\left(h^{\prime \prime}\right)_{z} \cdot r_{z, 0}\left(a^{\prime \prime} \otimes b^{\prime \prime}\right)\right) \\
&=h_{z} \cdot\left(\sum(-1)^{a^{\prime \prime} \tilde{b}^{\prime}}\left(\mathcal{E}_{z}(a)^{\prime}\right) \pi_{T}\left(b^{\prime}\right) r_{z, 0}\left((a)^{\prime \prime} \otimes b^{\prime \prime}\right)\right)=h_{z} \cdot\left(Y(a, z) \pi_{T}(b)\right) .
\end{aligned}
$$

One of the main advantages of the bicharacter construction is that there are explicit formulas for all the analytic continuation maps $X_{z_{1}, \ldots, z_{n}}$ in terms of the bicharacter, similar to the formula (IV.21). We will start with the formula for $X_{z_{1}, z_{2}, z_{3}}$ as it is needed also for the Operator Product Expansions. 
Definition IV.27 (Three-variable fields from a bicharacter) Let $V, W, \mathcal{E}_{z}, \pi_{T}: V \rightarrow W$ be as above. Let $a, b, c$ be arbitrary homogeneous elements of the super space $V$. Define the three variable field

$$
X_{z_{1}, z_{2}, z_{3}}: V^{\otimes 3} \rightarrow W\left[\left[z_{1}, z_{2}, z_{3}\right]\right] \otimes \mathbf{F}^{N}\left(z_{1}, z_{2}, z_{3}\right),
$$

by

$$
X_{z_{1}, z_{2}, z_{3}}(a \otimes b \otimes c)=\sum(-1)^{f(\tilde{a}, \tilde{b}, \tilde{c})} \mathcal{E}_{z_{1}} a^{(1)} \mathcal{E}_{z_{2}} b^{(1)} \mathcal{E}_{z_{3}} c^{(1)} r_{z_{1}, z_{2}}\left(a^{(2)} \otimes b^{(2)}\right) r_{z_{1}, z_{3}}\left(a^{(3)} \otimes c^{(2)}\right) r_{z_{2}, z_{3}}\left(b^{(3)} \otimes c^{(3)}\right),
$$

where $f(\tilde{a}, \tilde{b}, \tilde{c})=b^{(\tilde{3})}\left(\tilde{c^{(1)}}+c^{\tilde{(2)}}\right)+\left(\tilde{a^{(2)}}+a^{\tilde{(3)}}\right)\left(b^{\tilde{(1)}}+c^{\tilde{(1)}}\right)+a^{\tilde{(3)}}{b^{(2)}}^{\tilde{(2)}} b^{(2)} c^{(\tilde{1})}$. Here as usual we denote $\Delta^{2}(a)=a^{(1)} \otimes a^{(2)} \otimes a^{(3)}$ for any $a \in V$. The map $X_{z_{1}, z_{2}, z_{3}}$ is extended to the whole of $V$ by linearity.

Lemma IV.28 (n= $\mathbf{3}$ Analytic continuation) Let $V, W, \mathcal{E}_{z}, \pi_{T}: V \rightarrow W$ be as above. We have for any $a, b, c \in V$

$$
i_{z_{1}, z_{2}, z_{3}} X_{z_{1}, z_{2}, z_{3}}(a \otimes b \otimes c)=Y\left(a, z_{1}\right) Y\left(b, z_{2}\right) \mathcal{E}_{z_{3}} c=Y\left(a, z_{1}\right) Y\left(b, z_{2}\right) Y\left(c, z_{3}\right) 1 .
$$

Proof: Again from the "compatibility with bicharacters" and the "grouplike" property of the exponential map, and Definition IV.22 we have

$$
\begin{aligned}
& i_{z_{1}, z_{2}, z_{3}} X_{z_{1}, z_{2}, z_{3}}(a \otimes b \otimes c) \\
& =\sum(-1)^{f(\tilde{a}, \tilde{b}, \tilde{c})} \mathcal{E}_{z_{1}} a^{(1)} \mathcal{E}_{z_{2}} b^{(1)} \mathcal{E}_{z_{3}} c^{(1)} \cdot i_{z_{1}, z_{2}} r_{z_{1}, z_{2}}\left(a^{(2)} \otimes b^{(2)}\right) i_{z_{1}, z_{3}} r_{z_{1}, z_{3}}\left(a^{(3)} \otimes c^{(2)}\right) i_{z_{2}, z_{3}} r_{z_{2}, z_{3}}\left(b^{(3)} \otimes c^{(3)}\right) \\
& =\sum(-1)^{f(\tilde{a}, \tilde{b}, \tilde{c})} \mathcal{E}_{z_{1}} a^{(1)} \mathcal{E}_{z_{2}} b^{(1)} \mathcal{E}_{z_{3}} c^{(1)} \cdot r_{z_{1}, 0}\left(a^{(2)} \otimes \mathcal{E}_{z_{2}} b^{(2)}\right) r_{z_{1}, 0}\left(a^{(3)} \otimes \mathcal{E}_{z_{3}} c^{(2)}\right) r_{z_{2}, 0}\left(b^{(3)} \otimes \mathcal{E}_{z_{3}} c^{(3)}\right. \\
& =\sum(-1)^{\left(b^{\prime \prime}\right)^{\prime \prime}\left(\tilde{c^{\prime}}+\left(c^{\prime \prime}\right)^{\prime}\right)+\left(\left(a^{\prime \prime}\right)^{\prime}+\left(a^{\prime \prime}\right)^{\prime \prime}\right)\left(\tilde{b^{\prime}}+\tilde{c}^{\prime}\right)+\left(b^{\prime \prime}\right)^{\prime} c^{\prime}+\left(a^{\prime \prime}\right)^{\prime \prime}\left(b^{\prime \prime}\right)^{\prime}} \mathcal{E}_{z_{1}} a^{\prime} \mathcal{E}_{z_{2}} b^{\prime} \mathcal{E}_{z_{3}} c^{\prime} \cdot \\
& \left.\quad \cdot r_{z_{1}, 0}\left(\left(a^{\prime \prime}\right)^{\prime} \otimes\left(\mathcal{E}_{z_{2}}\left(b^{\prime \prime}\right)^{\prime}\right)\right) r_{z_{1}, 0}\left(\left(a^{\prime \prime}\right)^{\prime \prime} \otimes \mathcal{E}_{z_{3}}\left(c^{\prime \prime}\right)^{\prime}\right)\right) r_{z_{2}, 0}\left(\left(b^{\prime \prime}\right)^{\prime \prime} \otimes \mathcal{E}_{z_{3}}\left(c^{\prime \prime}\right)^{\prime \prime}\right. \\
& =\sum(-1)^{\left(b^{\prime \prime}\right)^{\prime \prime}\left(\tilde{c^{\prime}}+\left(c^{\prime \prime}\right)^{\prime}\right)+a^{\prime \prime} \tilde{b}^{\prime}+a^{\prime \prime} c^{\prime}+\left(b^{\prime \prime}\right)^{\prime} \tilde{c}^{\prime}} \mathcal{E}_{z_{1}} a^{\prime} \mathcal{E}_{z_{2}} b^{\prime} \mathcal{E}_{z_{3}} c^{\prime} \cdot r_{z_{1}, 0}\left(a^{\prime \prime} \otimes\left(\mathcal{E}_{z_{2}}\left(\left(b^{\prime \prime}\right)^{\prime}\right)\left(\mathcal{E}_{z_{3}} c^{\prime \prime}\right)^{\prime}\right)\right) r_{z_{2}, 0}\left(\left(b^{\prime \prime}\right)^{\prime \prime} \otimes\left(\mathcal{E}_{z_{3}} c^{\prime \prime}\right)^{\prime \prime}\right) \\
& =Y\left(a, z_{1}\right) Y\left(b, z_{2}\right) \mathcal{E}_{z_{3}} c .
\end{aligned}
$$

Similar formulas can be derived for any $X_{z_{1}, \ldots, z_{n}}, n \in \mathbb{N}$. Before we give those, as a corollary of the formula above we will derive a formula for Operator Product Expansions (OPEs). Let $r$ be a $H_{T_{\epsilon}}^{N} \otimes H_{T_{\epsilon}}^{N}$-covariant bicharacter on $V$, with values in $\mathbf{F}^{N}(z, w)^{+, w}$. For any $a, b \in V$ the bicharacter $r_{z, w}(a \otimes b)$ is just a function of $z$ and $w$ in $\mathbf{F}^{N}(z, w)$ and can be expanded as a Laurent series around $z=\epsilon^{i} w$ for any $i=0,1, \ldots, N-1$ : $r_{z, w}(a \otimes b)=\sum_{l=0}^{M_{a, b}-1} \frac{f_{a, b}^{i, l}}{\left(z-\epsilon^{i} w\right)^{l+1}}+r e g$. We denote by $M_{a, b}$ the order of the pole at $z=\epsilon^{i} w$ and note that $f_{a, b}^{i, l}=f_{a, b}^{i, l}(w)$ is a function only of $w$.

Recall we usually omit writing the indexing in $\triangle(a)=\sum_{p} a_{p}^{\prime} \otimes a_{p}^{\prime \prime}$, and write it just as $\triangle(a)=\sum a^{\prime} \otimes a^{\prime \prime}$ to unclutter notation, but this summation is always implicitly present.

Theorem IV.29 (Bicharacter formula for the Residues) Let $V, W, \mathcal{E}_{z}$ and $\pi_{T}$ be as above. Let $r$ be a $H_{T_{\epsilon}}^{N} \otimes H_{T_{\epsilon}}^{N}$-covariant bicharacter on $V$, with values in $\mathbf{F}^{N}(z, w)$, denote $M_{p q}=M_{a_{p}^{\prime \prime}, b_{q}^{\prime \prime}}$. For any homogeneous $a, b \in V$ and any $0 \leq k \leq M_{p q}-1$ we have

$$
\operatorname{Res}_{z=\epsilon^{i} w} X_{z, w, 0}(a \otimes b \otimes c)\left(z-\epsilon^{i} w\right)^{k} d z=\sum_{p, q} \sum_{l=k}^{M_{p q}-1}(-1)^{\tilde{a}^{\prime \prime} \tilde{b}^{\prime}} f_{a^{\prime \prime}, b^{\prime \prime}}^{i, l} Y\left(\left(T_{\epsilon}^{i} D^{(l-k)} a^{\prime}\right) \cdot b^{\prime}, w\right) \pi_{T}(c) .
$$

Proof: By using coassociativity and cocommutativity we have from IV.27

$$
\begin{aligned}
& \left.X_{z, w, 0}(a \otimes b \otimes c)=\sum_{p, q, r}(-1)^{\left(b^{\prime \prime}\right.}\right)^{\prime \prime}\left(\tilde{c^{\prime}}+\left(\tilde{c^{\prime \prime}}\right)^{\prime}\right)+\left(\left(a^{\tilde{\prime}}\right)^{\prime}+\left(a^{\tilde{\prime}}\right)^{\prime \prime}\right)\left(\tilde{b^{\prime}}+\tilde{c^{\prime}}\right)+\left(a^{\tilde{\prime}}\right)^{\prime \prime}\left(b^{\tilde{\prime}}\right)^{\prime}+\left(b^{\tilde{\prime}}\right)^{\prime} \tilde{c}^{\prime} . \\
& \left.\left.\cdot\left(\mathcal{E}_{z} a^{\prime}\right)\left(\mathcal{E}_{w} b^{\prime}\right) \pi_{T}\left(c^{\prime}\right) r_{z, w}\left(\left(a^{\prime \prime}\right)^{\prime} \otimes\left(b^{\prime \prime}\right)^{\prime}\right)\right) r_{z, 0}\left(\left(a^{\prime \prime}\right)^{\prime \prime} \otimes c^{\prime \prime}\right)^{\prime}\right) r_{w, 0}\left(\left(b^{\prime \prime}\right)^{\prime \prime} \otimes\left(c^{\prime \prime}\right)^{\prime \prime}\right)
\end{aligned}
$$

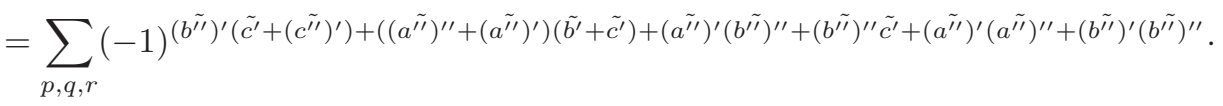

$$
\begin{aligned}
& \left.\left.\cdot\left(\mathcal{E}_{z} a^{\prime}\right)\left(\mathcal{E}_{w} b^{\prime}\right) \pi_{T}\left(c^{\prime}\right) r_{z, w}\left(\left(a^{\prime \prime}\right)^{\prime \prime} \otimes\left(b^{\prime \prime}\right)^{\prime \prime}\right)\right) r_{z, 0}\left(\left(a^{\prime \prime}\right)^{\prime} \otimes c^{\prime \prime}\right)^{\prime}\right) r_{w, 0}\left(\left(b^{\prime \prime}\right)^{\prime} \otimes\left(c^{\prime \prime}\right)^{\prime \prime}\right) \\
& =\sum_{p, q, r}(-1)^{\left.\left(b^{\prime}\right)^{\prime \prime}\left(\tilde{c^{\prime}}+\left(c^{\tilde{\prime}}\right)^{\prime}\right)+\left(\tilde{a^{\prime \prime}}+\left(a^{\prime}\right)^{\prime \prime}\right)\left(\left(b^{\prime}\right)^{\prime}+\tilde{c^{\prime}}\right)+\left(a^{\tilde{\prime}}\right)^{\prime \prime} \tilde{b}^{\tilde{\prime}}+\tilde{b}^{\prime \prime} \tilde{c}^{\prime}+\left(a^{\prime}\right)^{\prime \prime} \tilde{a}^{\tilde{\prime}}+(\tilde{b})^{\prime \prime}\right)^{\prime \prime}} \mathcal{E}_{z}\left(a^{\prime}\right)^{\prime} \mathcal{E}_{w}\left(b^{\prime}\right)^{\prime} . \\
& \left.\cdot \pi_{T}\left(c^{\prime}\right) \cdot r_{z, w}\left(a^{\prime \prime} \otimes b^{\prime \prime}\right)\right) r_{z, 0}\left(\left(a^{\prime}\right)^{\prime \prime} \otimes\left(c^{\prime \prime}\right)^{\prime}\right) r_{w, 0}\left(\left(b^{\prime}\right)^{\prime \prime} \otimes\left(c^{\prime \prime}\right)^{\prime \prime}\right) .
\end{aligned}
$$


Note that $r_{z, 0}\left(\left(a^{\prime}\right)^{\prime \prime} \otimes\left(c^{\prime \prime}\right)^{\prime}\right)$ is nonsingular at $z=\epsilon^{i} w$, and therefore can be expanded in a power series in $\left(z-\epsilon^{i} w\right)$ :

$$
\begin{aligned}
r_{z, 0}\left(\left(a^{\prime}\right)^{\prime \prime} \otimes\left(c^{\prime \prime}\right)^{\prime}\right) & \left.\left.\left.\left.=\sum_{j \geq 0}\left(\left(\partial_{z}\right)^{(j)} r_{z, 0}\left(\left(a^{\prime}\right)^{\prime \prime} \otimes c^{\prime \prime}\right)^{\prime}\right)\right)\right)\left.\right|_{z=\epsilon^{i} w}\left(z-\epsilon^{i} w\right)^{j}=\sum_{j \geq 0} r_{\epsilon^{i} w, 0}\left(D^{(j)}\left(a^{\prime}\right)^{\prime \prime} \otimes c^{\prime \prime}\right)^{\prime}\right)\right)\left(z-\epsilon^{i} w\right)^{j} \\
& \left.\left.\left.\left.=\sum_{j \geq 0} r_{w, 0}\left(T_{\epsilon}^{i} D^{(j)}\left(a^{\prime}\right)^{\prime \prime} \otimes c^{\prime \prime}\right)^{\prime}\right)\right)\left(z-\epsilon^{i} w\right)^{j}=\sum_{j \geq 0} \epsilon^{-i j} r_{w, 0}\left(D^{(j)} T_{\epsilon}^{i}\left(a^{\prime}\right)^{\prime \prime} \otimes c^{\prime \prime}\right)^{\prime}\right)\right)\left(z-\epsilon^{i} w\right)^{j}
\end{aligned}
$$

Next we use the modified expansion property of the exponential map, see lemma IV.18, and the fact that $f_{a^{\prime \prime}, b^{\prime \prime}}^{l}=0$ unless $\tilde{a^{\prime \prime}}=\tilde{b^{\prime \prime}}$, as the bicharacters are even.

$$
\begin{aligned}
& \operatorname{Res}_{z=\epsilon^{i} w} X_{z, w, 0}(a \otimes b \otimes c)\left(z-\epsilon^{i} w\right)^{k}
\end{aligned}
$$

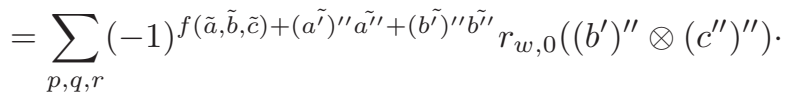

$$
\begin{aligned}
& \left.\left.\cdot \operatorname{Res}_{z=\epsilon^{i} w}\left(\left(\mathcal{E}_{z}\left(a^{\prime}\right)^{\prime}\right)\left(\mathcal{E}_{w}\left(b^{\prime}\right)^{\prime}\right) \pi_{T}\left(c^{\prime}\right)\left(\sum_{j} \epsilon^{-i j} r_{w, 0}\left(D^{(j)} T_{\epsilon}^{i}\left(a^{\prime}\right)^{\prime \prime} \otimes\left(c^{\prime \prime}\right)^{\prime}\right)\right)\left(z-\epsilon^{i} w\right)^{j+k}\right) \cdot r_{z, w}\left(a^{\prime \prime} \otimes b^{\prime \prime}\right)\right)\right) \\
& =\sum_{p, q, r}(-1)^{f(\tilde{a}, \tilde{b}, \tilde{c})+\left(a^{\prime}\right)^{\prime \prime} \tilde{a^{\prime \prime}}+\left(b^{\prime}\right)^{\prime \prime} \tilde{b}^{\prime \prime}} r_{w, 0}\left(\left(b^{\prime}\right)^{\prime \prime} \otimes\left(c^{\prime \prime}\right)^{\prime \prime}\right) . \\
& \left.\left.\cdot \operatorname{Res}_{z=\epsilon^{i} w}\left(\sum_{n, j \geq 0} \epsilon^{-i(n+j)}\left(z-\epsilon^{i} w\right)^{n+j+k} \mathcal{E}_{w}\left(D^{(n)} T a^{\prime}\right)^{\prime}\right)\left(\mathcal{E}_{w}\left(b^{\prime}\right)^{\prime}\right) \pi_{T}\left(c^{\prime}\right) \cdot r_{w, 0}\left(D^{(j)}\left(a^{\prime}\right)^{\prime \prime} \otimes\left(c^{\prime \prime}\right)^{\prime}\right) r_{z, w}\left(a^{\prime \prime} \otimes b^{\prime \prime}\right)\right)\right)
\end{aligned}
$$

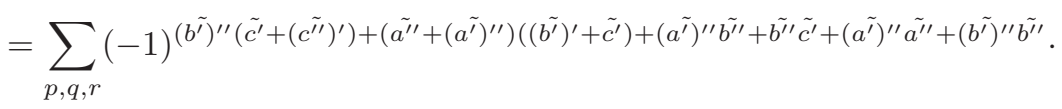

$$
\begin{aligned}
& \left.\left.\left.\left.\left.\cdot \sum_{l=k}^{M_{p q}-1} \mathcal{E}_{w}\left(\left(D^{(l-k)} T a^{\prime}\right)^{\prime}\right)\left(\mathcal{E}_{w}\left(b^{\prime}\right)^{\prime}\right) \pi_{T}\left(c^{\prime}\right) r_{w, 0}\left(D^{(l-k)}\left(T a^{\prime}\right)^{\prime \prime} \otimes\left(c^{\prime \prime}\right)^{\prime}\right)\right) \cdot r_{w, 0}\left(\left(b^{\prime}\right)^{\prime \prime} \otimes c^{\prime \prime}\right)^{\prime \prime}\right)\right)\right)\right) f_{a^{\prime \prime}, b^{\prime \prime}}^{k} \\
& =\sum_{p, q, r}(-1)^{\left(b^{\prime}\right)^{\prime \prime}} \tilde{c^{\prime}}+\tilde{a^{\prime \prime}}\left(\left(b^{\prime}\right)^{\prime}+\tilde{c^{\prime}}\right)+\left(a^{\prime}\right)^{\prime \prime} \tilde{c^{\prime}}+\left(a^{\prime}\right)^{\prime \prime} \tilde{b}^{\prime \prime}+\tilde{b^{\prime \prime}} \tilde{c^{\prime}}+\left(a^{\prime}\right)^{\prime \prime} \tilde{a^{\prime \prime}}+\left(b^{\prime}\right)^{\prime \prime} \tilde{b}^{\prime \prime} . \\
& \cdot\left(\sum_{l=k}^{M_{p q}-1}\left(\mathcal{E}_{w}\left(\left(D^{(l-k)} T a^{\prime}\right)\left(b^{\prime}\right)\right)^{\prime}\right) \pi_{T}\left(c^{\prime}\right) r_{w, 0}\left(\left(D^{(l-k)} T a^{\prime} b^{\prime}\right)^{\prime \prime} \otimes c^{\prime \prime}\right) f_{a^{\prime \prime}, b^{\prime \prime}}^{l}\right) \\
& =\sum_{p, q, r} \sum_{l=k}^{M_{p q}-1}(-1)^{\left(\left(b^{\prime}\right)^{\prime \prime}+\left(\tilde{a^{\prime}}\right) \tilde{c^{\prime}}+\tilde{a^{\prime \prime}} \tilde{b}^{\prime}\right.} \mathcal{E}_{w}\left(\left(\left(D^{(l-k)} a^{\prime}\right)\left(b^{\prime}\right)\right)^{\prime} \pi_{T}\left(c^{\prime}\right)\right) \cdot r_{w, 0}\left(\left(D^{(l-k)} T a^{\prime} b^{\prime}\right)^{\prime \prime} \otimes c^{\prime \prime}\right) f_{a^{\prime \prime}, b^{\prime \prime}}^{k} \\
& =\sum_{p, q} \sum_{l=k}^{M_{p q}-1}(-1)^{\tilde{a^{\prime \prime}} \tilde{b}^{\prime}} f_{a^{\prime \prime}, b^{\prime \prime}}^{l} Y\left(\left(D^{(l-k)} T a^{\prime}\right) \cdot b^{\prime}, w\right) \pi_{T}(c)
\end{aligned}
$$

Corollary IV.30 Let $V, W, r$ be as above, let again $M_{p q}=M_{a_{p}^{\prime \prime}, b_{q}^{\prime \prime}}$. For any $a, b \in V$ we have

$$
Y(a, z) Y(b, w)=i_{z, w} \sum_{p, q} \sum_{k=0}^{M_{p q}-1} \frac{\sum_{l=M_{p, q}-1-k}^{M_{p, q}-1}(-1)^{\tilde{a^{\prime \prime} b^{\prime}}} f_{a^{\prime \prime}, b^{\prime \prime}}^{i, l} Y\left(\left(T_{\epsilon}^{i} D^{(l-k)} a^{\prime}\right) \cdot b^{\prime}, w\right)}{\left(z-\epsilon^{i} w\right)^{k+1}}+\operatorname{Reg}_{(z, w)}^{\epsilon^{i}}(a \otimes b)
$$

The term $\operatorname{Reg}_{(z, w)}^{\epsilon^{i}}(a \otimes b)$ denotes the regular part in the Laurent expansion above, it depends on $a, b \in V, z$ and $w$, and $i \in\{0,1, \ldots N-1\}$.

Note that $\operatorname{Reg}_{(z, w)}^{\epsilon^{i}}(a \otimes b)$ is non-singular for $z=\epsilon^{i} w$, but it can still be singular at $z=\epsilon^{j} w$ for $j \neq i$, and thus $\operatorname{Reg}_{(z, w)}^{\epsilon^{i}}(a \otimes b)$ can potentially differ from the normal ordered product $: a(z) b(w):$. On the other hand, as the normal ordered product (II.9) is the regular part of an OPE of two fields, it is clear that if we have a single pole at $z=\epsilon^{i} w$, then $\operatorname{Reg}_{(z, w)}^{\epsilon^{i}}(a \otimes b)=: a(z) b(w)$ :. (For proof of that the normal ordered product is the regular part of the OPE of two fields in this more general context, see [ACJ13]). The above formula simplifies in the case of a simple pole: 
Corollary IV.31 (Bicharacter formula for OPEs for simple poles) Let $V, W, r$ be as above, and let $a, b \in V$ are such that the bicharacters $r_{z, w}\left(a^{\prime \prime} \otimes b^{\prime \prime}\right)$ have at most simple poles at each $a^{\prime \prime}, b^{\prime \prime}$. Then

$$
Y(a, z) Y(b, w)=i_{z, w} \sum_{p, q} \sum_{i}(-1)^{\tilde{a}^{\prime \prime} \tilde{b}^{\prime}} f_{a^{\prime \prime}, b^{\prime \prime}}^{i, 0} \frac{Y\left(\left(T^{i} a^{\prime}\right) \cdot b^{\prime}, w\right)}{z-\epsilon^{i} w}+: a(z) b(w): .
$$

We derived formulas for the analytic continuations of products of two fields, as well as formulas for the OPEs and normal order products via the bicharacter. These formulas always hold for any $H_{T_{\epsilon}}^{N} \otimes H_{T_{\epsilon}}^{N}$-covariant bicharacter on $V$, but if we want the vertex operators given by these formulas to satisfy all the axioms for a twisted vertex algebra the restriction that remains is the shift restriction, see remark III.3. Hence we need to impose the following restriction on the bicharacter:

Definition IV.32 (Shift restricted bicharacter) Let $r$ be a $H_{T_{\epsilon}}^{N} \otimes H_{T_{\epsilon}}^{N}$-covariant bicharacter on V, with values in $\mathbf{F}^{N}(z, w)$. Let as above $f_{a, b}^{i, l}$ stands for the coefficient in the expansion

$$
r_{z, w}(a \otimes b)=\sum_{l=0}^{M-1} \frac{f_{a, b}^{i, l}(w)}{\left(z-\epsilon^{i} w\right)^{l+1}}+\sum_{l=0}^{\infty} f_{a, b}^{i,-l-1}(w) \cdot\left(z-\epsilon^{i} w\right)^{l}
$$

as a Laurent series in $\left(z-\epsilon^{i} w\right)$. We call the bicharacter $r$ shift-restricted if for any $a, b \in V \quad f_{a, b}^{i, l}=c_{a, b}^{i, l} \cdot w^{s_{a, b}^{i, l}}$, where $c_{a, b}^{i, l}$ is a constant, $c_{a, b}^{i, l} \in \mathbb{C}$, and $s_{a, b}^{i, l} \in \mathbb{Z}$ such that $\left|s_{a, b}^{i, l}\right| \leq(N-1)(l+1)$.

Examples of shift-restricted bicharacters are given by functions in $\mathbf{F}^{N}(z, w)$ which have separately homogeneous numerators and denominators.

Lemma IV.33 (Bicharacter formula for the normal order product for simple poles) $L e t V, W, r$ be as above, and let $a, b \in V$ are such that the bicharacters $r_{z, w}\left(a_{k}^{\prime \prime} \otimes b_{l}^{\prime \prime}\right)$ have at most simple poles at each $a_{k}^{\prime \prime}, b_{l}^{\prime \prime}$. Then the normal order product : $Y(a, z) Y(b, z)$ : of the fields $Y(a, z)$ and $Y(b, w)$ is given by

$$
: Y(a, z) Y(b, z):=\sum_{i} \sum_{k, l} c_{a_{k}^{\prime \prime}, b_{l}^{\prime \prime}}^{i,-1}(-1)^{\tilde{a}_{k}^{\prime \prime}}{\tilde{b_{l}^{\prime}}}^{\prime} Y\left(a_{k}^{\prime} \cdot b_{l}^{\prime}, z\right)
$$

We want to finish this section by giving the general formulas for multivariable fields and analytic continuation of products of fields via bicharacter. Recall the extended Sweedler notation for an element $a$ in a commutative and cocommutative Hopf algebra, $n \in \mathbb{N}, n \geq 2$ :

$$
\triangle^{n-1}(a)=\sum_{s} a_{s}^{(1)} \otimes a_{s}^{(2)} \otimes \ldots \otimes a_{s}^{(n)},
$$

which again we will often write omitting the index $s$ to shorten the notation.

Notation IV.34 (Coproduct matrices)

Let $a_{1}, a_{2}, \ldots, a_{n}$ be $n$ elements of a commutative and cocommutative Hopf algebra. We can arrange the terms of the l-coproducts of these elements as sets of $n$ by $(l+1)$ matrices $M_{\Delta^{l}}^{\vec{k}}\left(a_{1}, a_{2}, \ldots, a_{n}\right)=\left(\left(a_{i}^{(j)}\right)_{\vec{k}}\right)_{j=1}^{l+1}$, where $\vec{k}=\left(k_{1}, k_{2}, \ldots, k_{n}\right)$ is the coproduct index.

Note that this is not one matrix, but a set of matrices, indexed by $\vec{k}$, with cardinality of the set dependent on the coproducts of $a_{1}, a_{2}, \ldots, a_{n}$. Since we are going to encounter a lot of sign contributions, we introduce the following notation:

Notation IV.35 (Sign notation) Let $M=\left(m_{i j}\right)_{i, j=1}^{n}$ be an $n$ by $n$ matrix with elements $m_{i j} \in V$. Let $\operatorname{sign}(M)=\operatorname{sign}\left(\left(m_{i j}\right)_{i, j=1}^{n}\right)$ denote the following sign value:

$$
\operatorname{sign}(M)=(-1)^{\sum_{i=2}^{n} \sum_{j=1}^{i-1} \sum_{k=2}^{n} \tilde{m}_{i 1} \tilde{m}_{j k}} \cdot(-1)^{\sum_{i=2}^{n} \sum_{j=2}^{n}\left(\sum_{k<i} \sum_{l \geq i+j-k} \tilde{m}_{i j} \tilde{m}_{k l}\right)} .
$$

If $l=n-1$ in the coproduct matrices above, $M_{\Delta^{n-1}}^{\vec{k}}\left(a_{1}, a_{2}, \ldots, a_{n}\right)$ are square matrices, and we can calculate $\operatorname{sign}\left(M_{\Delta^{n-1}}^{\vec{k}}\left(a_{1}, a_{2}, \ldots, a_{n}\right)\right)$ for each one. 
Example IV.36 Let $M=\mathbb{C}\{\phi\}$, as in exampleIV.12, $\phi$ is odd. We have $\Delta(\phi)=\phi \otimes 1+1 \otimes \phi$. Thus there are two matrices $M_{\Delta}^{\vec{k}}(\phi, 1), \vec{k} \in\{(1,1),(2,1)\}$ :

$M_{\Delta}^{(1,1)}(\phi, 1)=\left(\begin{array}{cc}\phi & 1 \\ 1 & 1\end{array}\right), \quad M_{\Delta}^{(2,1)}(\phi, 1)=\left(\begin{array}{cc}1 & \phi \\ 1 & 1\end{array}\right)$. Both have $\operatorname{sign}=1$

There are four matrices $M_{\Delta}^{\vec{k}}(\phi, \phi)$, i.e., $\vec{k} \in\{(1,1),(1,2),(2,1),(2,2)\}$ :

$M_{\Delta}^{(1,1)}(\phi, \phi)=\left(\begin{array}{cc}\phi & 1 \\ \phi & 1\end{array}\right), \quad M_{\Delta}^{(1,2)}(\phi, \phi)=\left(\begin{array}{cc}\phi & 1 \\ 1 & \phi\end{array}\right), \quad M_{\Delta}^{(2,1)}(\phi, \phi)=\left(\begin{array}{ll}1 & \phi \\ \phi & 1\end{array}\right), \quad M_{\Delta}^{(2,2)}(\phi, \phi)=\left(\begin{array}{cc}1 & \phi \\ 1 & \phi\end{array}\right)$.

We have $\operatorname{sign}\left(M_{\Delta}^{(1,1)}\right)=\operatorname{sign}\left(M_{\Delta}^{(1,2)}\right)=\operatorname{sign}\left(M_{\Delta}^{(2,2)}\right)=1, \operatorname{sign}\left(M_{\Delta}^{(2,1)}\right)=-1$.

Definition IV.37 (n-characters) Let $M$ be a commutative and cocommutative Hopf algebra, and let $r$ : $M \otimes M \rightarrow \mathbf{F}^{N}(z, w)$ be a super bicharacter on $M$. Let $a_{1}, a_{2}, \ldots, a_{n}$ be $n$ elements of $M$. Define an $n$ character $r_{n}: M^{\otimes n} \rightarrow \mathbf{F}^{N}\left(z_{1}, z_{2}, \ldots, z_{n}\right)$ by

$$
\begin{aligned}
r_{z_{1}, z_{2}, \ldots, z_{n}}\left(a_{1} \otimes a_{2} \otimes \ldots \otimes a_{n}\right)= & \sum_{\text {coproducts }} r_{z_{1}, z_{2}}\left(a_{1}^{(1)} \otimes a_{2}^{(1)}\right) r_{z_{1}, z_{3}}\left(a_{1}^{(2)} \otimes a_{3}^{(1)}\right) \ldots r_{z_{1}, z_{n}}\left(a_{1}^{(n-1)} \otimes a_{n}^{(1)}\right) . \\
& \cdot r_{z_{2}, z_{3}}\left(a_{2}^{(2)} \otimes a_{3}^{(2)}\right) \ldots r_{z_{2}, z_{n}}\left(a_{2}^{(n-1)} \otimes a_{n}^{(2)}\right) \ldots r_{z_{n-1}, z_{n}}\left(a_{n-1}^{(n-1)} \otimes a_{n}^{(n-1)}\right) .
\end{aligned}
$$

In particular, a tri-character $r_{3}: M \otimes M \otimes M \rightarrow \mathbf{F}^{N}\left(z_{1}, z_{2}, z_{3}\right)$ is given by

$$
\begin{aligned}
r_{z_{1}, z_{2}, z_{3}}\left(a_{1} \otimes a_{2} \otimes a_{3}\right) & =\sum_{\text {coproducts }} r_{z_{1}, z_{2}}\left(a_{1}^{\prime} \otimes a_{2}^{\prime}\right) r_{z_{1}, z_{3}}\left(a_{1}^{\prime \prime} \otimes a_{3}^{\prime}\right) r_{z_{2}, z_{3}}\left(a_{2}^{\prime \prime} \otimes a_{3}^{\prime \prime}\right) \\
& =\sum_{k_{1}, k_{2}, k_{3}} r_{z_{1}, z_{2}}\left(\left(a_{1}^{\prime}\right)_{k_{1}} \otimes\left(a_{2}^{\prime}\right)_{k_{2}}\right) r_{z_{1}, z_{3}}\left(\left(a_{1}^{\prime \prime}\right)_{k_{1}} \otimes\left(a_{3}^{\prime}\right)_{k_{3}}\right) r_{z_{2}, z_{3}}\left(\left(a_{2}^{\prime \prime}\right)_{k_{2}} \otimes\left(a_{3}^{\prime \prime}\right)_{k_{3}}\right) .
\end{aligned}
$$

Definition IV.38 (Multivariable fields from a bicharacter) Let $V, W, \mathcal{E}_{z}, \pi_{T}: V \rightarrow W$ be as above. Let $a_{1}, a_{2}, \ldots, a_{n}$ be any $n$ homogeneous elements of $V$. Define the $n$-variable field

$$
X_{z_{1}, z_{2}, \ldots, z_{n}}: V^{\otimes n} \rightarrow W\left[\left[z_{1}, z_{2}, \ldots, z_{n}\right]\right] \otimes \mathbf{F}^{N}\left(z_{1}, z_{2}, \ldots, z_{n}\right)
$$

by

$X_{z_{1}, z_{2}, \ldots, z_{n}}\left(a_{1} \otimes a_{2} \otimes \ldots \otimes a_{n}\right)=\sum_{\vec{k}} \operatorname{sign}\left(M_{\Delta^{n-1}}^{\vec{k}}\left(a_{1}, a_{2}, \ldots, a_{n}\right)\right) \mathcal{E}_{z_{1}} a_{1}^{\prime} \mathcal{E}_{z_{2}} a_{2}^{\prime} \ldots \mathcal{E}_{z_{n}} a_{n}^{\prime} \cdot r_{z_{1}, z_{2}, \ldots, z_{n}}\left(a_{1}^{\prime \prime} \otimes a_{2}^{\prime \prime} \otimes \ldots \otimes a_{n}^{\prime \prime}\right)$

Lemma IV.39 ( Analytic continuation) Let $V, W, \mathcal{E}_{z}, \pi_{T}: V \rightarrow W$ be as above. We have

$$
i_{z_{1}, z_{2}, \ldots, z_{n}} X_{z_{1}, z_{2}, \ldots, z_{n}}\left(a_{1} \otimes a_{2} \otimes \ldots \otimes a_{n}\right)=Y\left(a_{1}, z_{1}\right) Y\left(a_{2}, z_{2}\right) \ldots \mathcal{E}_{z_{n}} a_{n}=Y\left(a_{1}, z_{1}\right) Y\left(a_{2}, z_{2}\right) \ldots Y\left(a_{n}, z_{n}\right) 1
$$

for any $a_{1}, a_{2}, \ldots, a_{n} \in V$.

The proof is very similar to the proof of lemma IV.28 and we omit it.

\section{Remark IV.40 (Keeping track of sign contributions with variables)}

The use of the sign notation can be restated as follows: In the definition IV.38 one can think of the variables $z_{1}, z_{2}, \ldots, z_{n}$ as "attached" to the arguments $a_{1}, a_{2}, \ldots, a_{n}$ and correspondingly their coproducts. One then multiplies by a minus sign whenever an odd element with attached variable $z_{j}$ appears before ("transposes") another odd element with attached variable $z_{i}$, where $i<j$. There is no sign contribution unless both elements are odd.

We summarize the entire construction in the main bicharacter theorem:

Theorem IV.41 Let $M$ be a commutative cocommutative Hopf algebra, let $V$ be the free Leibnitz module $V=H_{T_{\epsilon}}^{N}(M), r$ be a shift-restricted $H_{T_{\epsilon}}^{N} \otimes H_{T_{\epsilon}}^{N}$-covariant symmetric bicharacter on $V$ with values in in $\mathbf{F}^{N}(z, w)^{+, w}, W=H_{D}(M)$ be the free $H_{D}$-Leibnitz sub-module-algebra of $V$. Let $\pi_{T}: V \rightarrow W$ be the projection map as in definition IV.15 and $Y$ be the field-state correspondence defined by (IV.22), via (IV.21). The set of data $\left(V, W, \pi_{T}, Y\right)$ constructed as above satisfies the definition of a twisted vertex algebra for any shift-restricted supercommutative $H_{T_{\epsilon}}^{N} \otimes H_{T_{\epsilon}}^{N}$-covariant bicharacter on $V$. 


\section{EXAMPLES OF TWISTED VERTEX ALGEBRAS BASED ON A BICHARACTER}

In most of the examples in the literature vertex operators are presented in terms of generating fields and commutation relations. With the bicharacter construction as we saw in the previous section (Theorem IV.41) one starts instead with the commutative cocommutative Hopf algebra $M$ and its free Leibnitz module $H_{T_{\epsilon}}^{N}(M)$; the OPEs and thus the commutation relations are then dictated by the choice of the bicharacter $r$. Moreover, for each commutative cocommutative Hopf algebra $M$ there are many choices of a symmetric bicharacter $r$, and so each such pair $(M, r)$ will give rise to a different twisted vertex algebra $\left(V, W, \pi_{T}, Y\right)$, even if the spaces $V$ and $W$ are the same as bialgebras - since the field-state correspondence $Y$ changes with the choice of a bicharacter. This is the case in particular for the fermionic sides of the B and the D-A correspondences: there the space of fields $V$ and the space of states $W$ coincide as free Leibnitz modules, but the generating fields for each are different, which results in highest weight modules for different Clifford algebras. Hence for the bicharacter construction examples are grouped based on the Hopf algebra $M$, i.e. one starts by keeping $M$ the same, but changing the bicharacter $r$ on $M$. We want to stress the fact that there is a variety of examples even after we fix the algebra $M$. In the following we will list the examples grouped by the underlying Hopf algebra $M$.

\section{A. Twisted vertex algebras based on $\mathbb{C}\{\phi\}$ and a choice of a bicharacter}

Fix $M=\mathbb{C}\{\phi\}$ from example IV.12, To define a $H_{T_{\epsilon}}^{N} \otimes H_{T_{\epsilon}}^{N}$-covariant bicharacter on $H_{T_{\epsilon}}^{N}(M)$, one is only allowed to chose $r_{z, w}(\phi \otimes \phi)$, as all the other values of the bicharacter on $H_{T_{\epsilon}}^{N}(\mathbb{C}\{\phi\})$ would be in turn determined by the covariance and the bicharacter properties (see section IVA). Thus a twisted vertex algebra $V$ based on $M=\mathbb{C}\{\phi\}$ will be determined entirely by a supersymmetric bicharacter value $r_{z, w}(\phi \otimes \phi)$.

Let us specialize further, and consider the case of $N=2$-twisted vertex algebra $(\epsilon=-1)$. From example IV.12, the free Leibnitz module $H_{T_{-1}}^{2}(\mathbb{C}\{\phi\})$ is isomorphic to $H_{D}(\mathbb{C}\{\phi, T \phi\})$. Before proceeding to specific examples (dependent on the value of $r_{z, w}(\phi \otimes \phi)$ ), we want to present a formula for the vacuum expectation values valid for any choice of $r_{z, w}(\phi \otimes \phi)$.

\section{B. Twisted vertex algebras based on $\mathbb{C}\{\phi\}$ : Pfaffian vacuum expectation values}

Let $\langle\mid\rangle: W \otimes W \rightarrow \mathbb{C}$ be a symmetric bilinear form on the space of states $W=H_{D}(\mathbb{C}\{\phi\})$. There is a very important concept of an invariant bilinear form on a vertex algebra, for details see for example Li94 and $\mathrm{Xu} 98$ ]. It is not our goal here to define a general invariant bilinear form for a twisted vertex algebra, but for our bicharacter construction we will require that any bilinear form is such that the vacuum vector $1=|0\rangle$ is orthogonal to all other generators of the algebra $W$ and has norm 1, i.e., $\langle 1 \mid 1\rangle=\langle\langle 0|| \mid 0\rangle\rangle=1$. By abuse of notation we will just write $\langle 0 \mid 0\rangle$ instead of $\langle\langle 0|| \mid 0\rangle\rangle$. We can extend this form to $W\left(\left(z_{1}, z_{2}, \ldots\right)\right) \otimes$ $W\left(\left(z_{1}, z_{2}, \ldots\right)\right) \rightarrow \mathbb{C}\left(\left(z_{1}, z_{2}, \ldots\right)\right)$ by bilinearity. The values $\left\langle 0\left|Y\left(a, z_{1}\right) Y\left(a, z_{2}\right) \ldots Y\left(a, z_{n}\right)\right| 0\right\rangle$ of the bilinear form are usually called vacuum expectation values.

Proposition V.1 Let $V$ be a twisted vertex algebra based on $M=\mathbb{C}\{\phi\}$ and a supersymmetric bicharacter $r$ (in particular, $V=H_{D}(\mathbb{C}\{\phi, T \phi\})$ and $\left.W=H_{D}(\mathbb{C}\{\phi\})\right)$. Denote by $\phi(z)$ the field $Y(\phi, z)$ produced by definition IV.22, via (IV.21). The following formula for the vacuum expectation values holds:

$$
\left\langle 0\left|\phi\left(z_{1}\right) \phi\left(z_{2}\right) \ldots \phi\left(z_{2 n}\right)\right| 0\right\rangle=i_{z} \operatorname{Pf}\left(r_{z_{i}, z_{j}}(\phi \otimes \phi)\right)_{i, j=1}^{2 n} .
$$

Here as usual Pf denotes the Pfaffian of an antisymmetric matrix and $i_{z}$ stands for the expansion $i_{z_{1}, z_{1}, \ldots, z_{2 n}}$.

Note that the matrix on the right-hand side is antisymmetric since the bicharacter $r$ is symmetric and $\phi$ is odd, i.e., $r_{z_{i}, z_{j}}(\phi \otimes \phi)=-r_{z_{j}, z_{i}}(\phi \otimes \phi)$.

Proof: To calculate the vacuum expectation values we calculate instead the vacuum expectation values of the analytic continuation $X_{z_{1}, z_{2}, \ldots, z_{2 n}}(\phi \otimes \phi \ldots \phi)$ by use of Lemma IV.39. Since $\phi$ is a primitive element, we have

$$
\triangle^{2 n}(\phi)=\phi \otimes 1 \otimes \ldots \otimes 1+1 \otimes \phi \otimes \ldots \otimes 1+1 \otimes 1 \otimes \phi \otimes \ldots \otimes 1+\ldots 1 \otimes 1 \otimes \ldots \otimes \phi .
$$

We need three observations: 
1. Since for the bilinear form the vacuum vector $1=|0\rangle$ spans an orthogonal subspace on its own (and in particular is orthogonal to $\phi$ and its descendants), the only contributions to the vacuum expectation values will come from the terms in the multivariable field where the coproducts have 1 as a first term; the other terms will not contribute. That forces us to work with the $(2 n)$-character $r_{z_{1}, z_{2}, \ldots, z_{2 n}}(\phi \otimes \ldots \otimes \phi)$.

2. No sign contribution will come from the first $(-1)$ factor of $\operatorname{sign}\left(M_{\Delta^{2 n-1}}^{\vec{k}}(\phi, \phi, \ldots, \phi)\right)$, as the only contributing matrices are those with the first columns consisting entirely of $1 \mathrm{~s}$ ( 1 is even).

3. Since $\phi$ is a primitive element we have $r_{z, w}(\phi \otimes 1)=r_{z, w}(1 \otimes \phi)=0$ for any bicharacter. Thus the only contributions in the $(2 n)$-character $r_{z_{1}, z_{2}, \ldots, z_{2 n}}(\phi \otimes \phi \otimes \ldots \otimes \phi)$ will come from the following situation: Consider a matrix $M_{\Delta^{2 n-1}}^{\vec{k}}(\phi, \phi, \ldots, \phi)$ with first column entirely consisting of 1 s. A nonzero summand in the $(2 n)$-character $r_{z_{1}, z_{2}, \ldots, z_{2 n}}(\phi \otimes \phi \otimes \ldots \otimes \phi)$ will be a product of nonzero bicharacter factors, and that happens when we have a sequence of either $(1,1)$ pairs (trivial, as $\left.r_{z, w}(1 \otimes 1)=1\right)$, or $(\phi, \phi)$ pairs (nontrivial). If there is a mixed pair $(1, \phi)$ or $(\phi, 1)$ as a factor in a summand, that summand will be 0 . So a nonzero summand will have exactly $n$ such nontrivial contributing pairs $(\phi, \phi)$, and each pair forms one bicharacter $r_{z_{i}, z_{j}}(\phi \otimes \phi)$. The sign contribution will come only from the nontrivial pairs.

Thus, we have

$$
\begin{aligned}
X_{z_{1}, z_{2}, \ldots, z_{2 n}}(\phi \otimes \phi \otimes \ldots \otimes \phi) \\
=\sum_{\text {coproducts }} \operatorname{sign}\left(M_{\Delta^{2 n-1}}^{\vec{k}}(\phi, \phi, \ldots, \phi)\right) \mathcal{E}_{z_{1}} \phi^{\prime} \mathcal{E}_{z_{2}} \phi^{\prime} \cdots \mathcal{E}_{z_{2 n}} \phi^{\prime} \cdot r_{z_{1}, \ldots, z_{2 n}}\left(\phi^{\prime \prime} \otimes \phi^{\prime \prime} \otimes \ldots \otimes \phi^{\prime \prime}\right) \\
=\sum_{\substack{\text { contr. } \\
\text { coproducts }}} \operatorname{sign}\left(M_{\Delta}^{\vec{k}}{ }^{2 n-1}(\phi, \phi, \ldots, \phi)\right) 1 \cdot r_{z_{1}, z_{2}, \ldots, z_{2 n}}(\phi \otimes \phi \otimes \ldots \otimes \phi)+\ldots \\
=\sum_{P} \epsilon(P) 1 \cdot r_{z_{i_{1}}, z_{i_{2}}}(\phi \otimes \phi) r_{z_{i_{3}}, z_{i_{4}}}(\phi \otimes \phi) \cdots r_{z_{i_{2 n-1}}, z_{i_{2 n}}}(\phi \otimes \phi)+\text { other terms. }
\end{aligned}
$$

The sum is over all permutations such that $i_{1}<i_{2}, i_{3}<i_{4}, \ldots, i_{2 n-1}<i_{2 n}, i_{1}<i_{3}<\cdots<i_{2 n-1}$. The sign contribution from any contributing matrix $\left.M_{\Delta^{2 n-1}}^{\vec{k}}(\phi, \phi, \ldots, \phi)\right)$ is precisely the sign of the corresponding permutation, since $\phi$ is odd (see remarkIV.40 and observation 3 above). That produces precisely the Pfaffian $\operatorname{Pf}\left(r_{z_{i}, z_{j}}(\phi \otimes \phi)\right)_{i, j=1}^{2 n}$.

Next we will consider examples of twisted vertex algebras arising from specific bicharacter values for $r_{z, w}(\phi \otimes$ $\phi)$ by use of Theorem IV.41

\section{Twisted vertex algebras based on $\mathbb{C}\{\phi\}$ : the neutral free fermion of type B}

We continue working with space of fields $V=H_{T}^{2}(\mathbb{C}\{\phi\}) \equiv H_{D}(\mathbb{C}\{\phi, T \phi\})$, and space of states $W=$ $H_{D}(\mathbb{C}\{\phi\})$. The projection map (recall definition IV.15) in this case is just the algebra homomorphism defined by $\pi_{T}(T \phi)=\phi$.

Let the covariant bicharacter $r^{B}: H_{D}(\mathbb{C}\{\phi, T \phi\}) \otimes H_{D}(\mathbb{C}\{\phi, T \phi\}) \rightarrow \mathbf{F}^{2}(z, w)^{+, w}$ be defined by

$$
r_{z, w}^{B}(\phi \otimes \phi)=\frac{z-w}{z+w}
$$

Note that the bicharacter $r^{B}$ is symmetric, as it is symmetric on the generator $\phi$, it is also shift-restricted, and has a simple single pole. Theorem IV.41 asserts that the pair $\left(\mathbb{C}\{\phi\}, r^{B}\right)$ will produce an example of a twisted vertex algebra of order 2 . We claim that this $\mathrm{N}=2$ twisted vertex algebra is the free fermion of type B. To prove it, we need to show first that the field $\phi^{B}(z)$ corresponding to the element $\phi$ via the field-state correspondence defined by (IV.22) coincides with the free fermion field of type B introduced in section IIIB (i.e., satisfies the OPE (III.6) ). We use corollary IV.31 to calculate the OPE of $\phi^{B}(z) \phi^{B}(w)$. The only singular bicharacter from any of the coproducts $\phi^{\prime \prime}$ and $\phi^{\prime \prime}$ is $r_{z, w}^{B}(\phi \otimes \phi)$. Thus

$$
\phi^{B}(z) \phi^{B}(w) \sim i_{z, w} \sum(-1)^{\tilde{\phi}^{\prime \prime} \tilde{\phi}^{\prime}} f_{\phi^{\prime \prime}, \phi^{\prime \prime}}^{1,0} \frac{Y\left(\left(T \phi^{\prime}\right) \cdot \phi^{\prime}, w\right)}{(z+w)} \sim i_{z, w}(-1)^{\tilde{\phi} \tilde{1}} f_{\phi, \phi}^{1,0} \frac{Y((T 1) \cdot 1, w)}{(z+w)} \sim-\frac{2 w \cdot 1}{z+w}
$$


Lemma V.2 The normal ordered product field $h(z)$ from (III.7) defined by $\frac{1}{4}\left(: \phi^{B}(z) \phi^{B}(-z):-1\right)$ corresponds to

$$
h(z)=\frac{1}{4} Y(\phi \cdot T \phi, z), \quad h=\frac{1}{4} h_{\phi}, \quad \text { where } h_{\phi}=\phi \cdot T \phi, \text { see Example IV.12 }
$$

Proof: From lemma IV.33

$$
\begin{aligned}
: \phi^{B}(z) \phi^{B}(-z): & =: \phi^{B}(z) T \phi^{B}(z):=\sum(-1)^{\tilde{\phi^{\prime \prime}} \tilde{\phi}^{\prime}} f_{\phi^{\prime \prime}, \phi^{\prime \prime}}^{1,-1} Y\left(\phi^{\prime} \cdot(T \phi)^{\prime}, z\right) \\
& =(-1)^{\tilde{\phi} \tilde{1}} f_{\phi, \phi}^{1,-1} Y(1 \cdot 1, z)+(-1)^{\tilde{\phi} \tilde{1}} f_{1,1}^{1,-1} Y(\phi \cdot T \phi, z)=1_{W}+Y(\phi \cdot T \phi, z) .
\end{aligned}
$$

To calculate the OPEs (III.8) one uses theorem IV.29 to get

$$
h_{\phi}(z) h_{\phi}(w) \sim+Y(1, w) r_{z, w}\left(h_{\phi} \otimes h_{\phi}\right) \sim \frac{8 z w\left(z^{2}+w^{2}\right)}{\left(z^{2}-w^{2}\right)^{2}} .
$$

Since the fermionic side of of the boson-fermion correspondence of type B is known, see DJKM82] and You89], we omit most of the calculations.

We have $T h=\frac{1}{4} T \phi \cdot \phi=-h$, and since $Y(T h, z)=Y(h,-z)$ from the transfer of action axiom of twisted vertex algebras, we have $Y(h,-z)=-Y(h, z)$ in the twisted vertex algebra. Hence we have only odd powers of $z$ in $Y(h, z)$, and the indexing in $h(z)=\sum_{n \in \mathbb{Z}} h_{2 n+1} z^{-2 n-1}$ is implied. The commutation relations $\left[h_{m}, h_{n}\right]=\frac{m}{2} \delta_{m+n, 0} 1$ for the twisted Heisenberg algebra $\mathcal{H}_{\mathbb{Z}+1 / 2}$ then follow from the OPE in a standard calculation.

The decomposition of the space of states $F_{B}=W=H_{D}(\mathbb{C}\{\phi\})$ into twisted Heisenberg modules, Lemma [II.11 was done first in [DJKM82] and You89], and the calculations using the bicharacter formulas (IV.22) and (IV.21) are also available.

To calculate the image of the generating field $\phi^{B}(z) \mapsto e^{\alpha}(z)$ we first calculate the $\operatorname{OPE} h(z) \phi^{B}(w)$. As all the poles are simple here, from corollary IV.31 we get

$$
h(z) \phi^{B}(w) \sim \frac{1}{4}\left(\frac{2 w}{z-w}+\frac{2 w}{z+w}\right) Y(\phi, w) \sim \frac{z w}{z^{2}-w^{2}} \phi^{B}(w) .
$$

From this OPE and the exact description of the split of $W$ into irreducible Heisenberg submodules, by use of the standard calculational lemmas (see for example Kac90 and Wak01]) we get that the exponential boson formula (III.10) holds for the field $\phi^{B}(w)$.

\section{Twisted vertex algebras based on $\mathbb{C}\{\phi\}$ : the neutral free fermion of type D-A}

As in the previous section the space of fields is $V=H_{T}^{2}(\mathbb{C}\{\phi\})$, and the space of states is $W=H_{D}(\mathbb{C}\{\phi\})$, with the same projection map. Let the bicharacter $r^{D}: H_{D}(\mathbb{C}\{\phi, T \phi\}) \otimes H_{D}(\mathbb{C}\{\phi, T \phi\}) \rightarrow \mathbf{F}^{2}(z, w)^{+, w}$ be given by

$$
r_{z, w}^{D}(\phi \otimes \phi)=\frac{1}{z-w} .
$$

The bicharacter $r^{D}$ is symmetric, it is shift-restricted, and has a simple single pole. By Theorem IV.41 the pair $\left(\mathbb{C}\{\phi\}, r^{D}\right)$ will produce an example of a twisted vertex algebra of order 2 . We claim that this twisted vertex algebra is the free neutral fermion of type D-A (see Section IIIC). Since this is the new example of the boson-fermion correspondence of type D-A, we will go carefully over the details.

We use Corollary IV.31 to calculate the OPE of $\phi^{D}(z) \phi^{D}(w)$. Since $\phi$ is primitive, $r_{z, w}^{D}(\phi \otimes 1)=r_{z, w}^{D}(1 \otimes \phi)=$ 0 , and the only nontrivial bicharacter from any of the coproducts $\phi^{\prime \prime}$ and $\phi^{\prime \prime}$ is $r_{z, w}^{D}(\phi \otimes \phi)$. Thus

$$
\phi^{D}(z) \phi^{D}(w) \sim i_{z, w} \sum(-1)^{\tilde{\phi}^{\prime \prime} \tilde{\phi}^{\prime}} f_{\phi^{\prime \prime}, \phi^{\prime \prime}}^{1,0} \frac{Y\left(\left(T \phi^{\prime}\right) \cdot \phi^{\prime}, w\right)}{(z-w)} \sim \frac{1}{z-w} .
$$

This OPE coincides with (III.11) and corresponds to the anticommutation relations:

$$
\left[\phi^{D}(z), \phi^{D}(w)\right]_{\dagger}=i_{z, w} \frac{1}{z-w}+i_{w, z} \frac{1}{w-z}=\left(i_{z, w}-i_{w, z}\right) \frac{1}{z-w} .
$$


Using the notation $\delta(z-w)=\left(i_{z, w}-i_{w, z}\right) \frac{1}{z-w}=\sum_{j \in \mathbb{Z}} z^{-j-1} w^{j}$ we can write

$$
\left[\phi^{D}(z), \phi^{D}(w)\right]_{\dagger}=\delta(z-w)
$$

which if we index the field $\phi^{D}(z)=\sum_{n \in \mathbf{Z}+\mathbf{1} / \mathbf{2}} \phi_{n}^{D} z^{-n-1 / 2}$ will give us the required anticommutation relations $\left[\phi_{m}^{D}, \phi_{n}^{D}\right]_{\dagger}=\delta_{m,-n} 1$. The field $\phi^{D}(z)$ is well known as the neutral free fermion (of type D). Since in the OPE of $\phi^{D}(z)$ the only pole is at $z=w$, it is immediate that $\phi^{D}(z)$ on its own will generate a super vertex algebra (see e.g. Kac98], KWY98], Wan99]). $\phi^{D}(z)$ cannot be bosonized on its own, but as a new ingredient allowed in twisted vertex algebras we consider another descendant of $\phi^{D}(z)$ - the field $T \phi^{D}(z)=\phi^{D}(-z)$. Using the language of delta functions (see e.g. Kac98, Wak01, ACJ13]) we get

$$
\left[T \phi^{D}(z), T \phi^{D}(w)\right]_{\dagger}=-\delta(z-w), \quad\left[T \phi_{m}^{D}, T \phi_{n}^{D}\right]_{\dagger}=-\delta_{m,-n} 1,
$$

Thus on its own each of the fields $\phi^{D}(z)$ and $T \phi^{D}(z)$ (without the other) will generate a super vertex algebra, but the two "glue together" to form a twisted vertex algebra. This resembles the gluing together of the two sheets of the square root Riemann surface.

Since in a twisted vertex algebra both the fields $\phi^{D}(z)$ and $T \phi^{D}(z)$ (and their descendants) are available, we can form the field

$$
h(z)=\frac{1}{2}: \phi^{D}(z) \phi^{D}(-z):=\frac{1}{2}: \phi^{D}(z) T \phi^{D}(z): .
$$

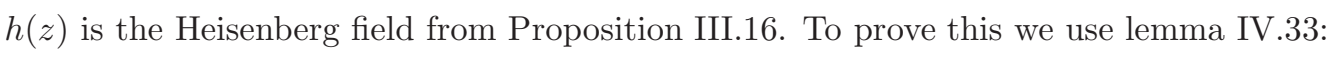

$$
\begin{aligned}
: \phi^{D}(z) T \phi^{D}(z): & =\sum(-1)^{\tilde{\phi}^{\prime \prime} \tilde{\phi}^{\prime}} f_{\phi^{\prime \prime}, \phi^{\prime \prime}}^{1,-1} Y\left(\phi^{\prime} \cdot(T \phi)^{\prime}, z\right)= \\
& =(-1)^{\tilde{\phi} \tilde{1}} f_{\phi, \phi}^{1,-1} Y(1 \cdot 1, z)+(-1)^{\tilde{\phi} \tilde{1}} f_{1,1}^{1,-1} Y(\phi \cdot T \phi, z)=0 \cdot 1_{W}+Y(\phi \cdot T \phi, z) .
\end{aligned}
$$

Thus the field $h(z)$ from (III.12) is actually the vertex operator $Y\left(\frac{1}{2} \phi \cdot T \phi, z\right)$ corresponding to the element $\frac{1}{2} h_{\phi}=\frac{1}{2} \phi \cdot T \phi$. To calculate the OPE of (III.13) we use theorem IV.29 We look at the coproduct :

$$
\triangle h_{\phi}=h_{\phi} \otimes 1+1 \otimes h_{\phi}+\phi \otimes T \phi-T \phi \otimes \phi .
$$

The first order poles in the OPE come from the $h^{\prime \prime}$ terms of the coproduct which have first order poles in their bicharacter, namely from $r_{z, w}(\phi \otimes T \phi)=-r_{z, w}(T \phi \otimes \phi)=\frac{1}{z+w}$ and from $r_{z, w}(\phi \otimes \phi)=-r_{z, w}(T \phi \otimes T \phi)=\frac{1}{z-w}$. The OPE coefficients in front of the first order poles then are:

for $\frac{1}{z-w}$ we get $Y(-\phi \cdot \phi+T \phi \cdot T \phi, w)$, which is zero as $\phi \cdot \phi=0=T \phi \cdot T \phi$, for $\frac{z-w}{z+w}$ we get $Y\left(-T \phi \cdot T \phi+T^{2} \phi \cdot \phi, w\right)$, which is zero as $T^{2} \phi=\phi$. Thus there are no first order poles in the OPEs of $h(z) h(w)$. The second order pole comes from

$$
\begin{aligned}
r_{z, w}\left(h_{\phi} \otimes h_{\phi}\right) & =r_{z, w}(\phi \cdot T \phi \otimes \phi \cdot T \phi)=-r_{z, w}(\phi \otimes \phi) r_{z, w}(T \phi \otimes T \phi)+r_{z, w}(\phi \otimes T \phi) r_{z, w}(T \phi \otimes \phi) \\
& =+\frac{1}{(z-w)^{2}}-\frac{1}{(z+w)^{2}}=\frac{4 z w}{\left(z^{2}-w^{2}\right)^{2}} .
\end{aligned}
$$

Further, we have $T h=\frac{1}{2} T \phi \cdot \phi=-h$, and since $Y(T h, z)=Y(h,-z)$ from the transfer of action axiom of twisted vertex algebras, we have $Y(h,-z)=-Y(h, z)$. Which means that we have only odd powers of $z$ in $Y(h, z)$, and we can index it $h(z)=\sum_{n \in \mathbb{Z}} h_{n} z^{-2 n-1}$. With this indexing, we have from the OPE:

$$
[h(z), h(w)]=\left(i_{z, w}-i_{w, z}\right) \frac{z w}{\left(z^{2}-w^{2}\right)^{2}}=\sum_{n \in \mathbb{Z}} n \frac{w^{2 n-1}}{z^{2 n+1}}=\frac{1}{4} \partial_{w}(\delta(z-w)+\delta(z+w)),
$$

which gives us the required commutation relations for the Heisenberg algebra $\mathcal{H}_{\mathbb{Z}}$ and proves Proposition III.16

Remark V.3 The field $h(z)$ has a very special property (which was also true for the B case), peculiar to twisted vertex algebras, and not possible in a super vertex algebra. $h(z)$ corresponds to the nonzero element $h$ in the space of fields of the twisted vertex algebra $V$, i.e., $h(z)=Y(h, z)$. But the projection of $h$ to the space of states is zero, as

$$
\pi_{T}(h)=\pi_{T}\left(\frac{1}{2} h_{\phi}\right)=\pi_{T}\left(\frac{1}{2} \phi \cdot T \phi\right)=\frac{1}{2} \phi \cdot \phi=0 .
$$


We now prove Proposition III.17 In order to decompose the space of states $F_{D}=W=H_{D}(\mathbb{C}\{\phi\})$ into Heisenberg submodules we need to identify the highest weight vectors in $W$. These are the elements $a_{\lambda}$ of $W$ such that $h(z) a_{\lambda}$ has only nonnegative powers of $z$. Let $a_{n}^{\text {even }}=\phi D^{(2)} \phi \cdots D^{(2 n)} \phi$, and denote as usual $\phi^{i}=D^{(i)} \phi$. We claim $a_{n}^{\text {even }}$ is a highest weight vector for $\mathcal{H}_{\mathbb{Z}}$. To prove that, we will be using the bicharacter formula (IV.22) and (IV.21). We can easily calculate the coproduct of $a_{n}^{\text {even: }}$

$$
\triangle\left(a_{n}^{\text {even }}\right)=\sum_{i}(-1)^{i-1} \phi \phi^{2} \cdots \widehat{\phi^{2 i}} \cdots \phi^{2 n} \otimes \phi^{2 i}+\sum_{i, j}(-1)^{i+j} \phi \cdots \widehat{\phi^{2 i}} \cdots \widehat{\phi^{2 j}} \cdots \phi^{2 n} \otimes \phi^{2 i} \phi^{2 j}+\ldots
$$

The only parts of the coproduct of consequence in this case are the parts with either single or quadratic terms in $\left(a_{n}^{\text {even }}\right)^{\prime \prime}$, as the bicharacter with any term from $h^{\prime \prime}$ will be 0 otherwise.

We first consider the even $a_{0}^{\text {even }}=1$ : We have

$$
h(z) 1=\mathcal{E}_{z} h=\frac{1}{2} e^{z D} \phi \cdot e^{-z D} \phi=\sum_{n \in \mathbb{Z}_{\geq 0}} z^{2 n+1} \sum_{p+q=2 n+1}(-1)^{q} D^{(p)} \phi D^{(q)} \phi=\sum_{n \in \mathbb{Z}_{\geq 0}} z^{2 n+1} \sum_{p+q=2 n+1}(-1)^{q} \phi^{p} \phi^{q},
$$

since we have $\sum_{p+q=\text { even }}(-1)^{q} D^{(p)} \phi D^{(q)} \phi=0$. Thus $a_{0}^{\text {even }}$ is a highest weight vector, with highest weight 0 (as $\left.h_{0} 1=0\right)$. Further, we have:

$$
\begin{gathered}
r_{z, 0}\left(\phi T \phi \otimes \phi^{i} \phi^{j}\right)=0 \quad \text { if } i, j \text { both even, or if } i, j \text { both odd } \\
r_{z, 0}\left(\phi T \phi \otimes \phi^{i} \phi^{j}\right)=-\frac{2}{z^{i+j+2}} \quad \text { if } i=\text { even, } j=\text { odd } \\
r_{z, 0}\left(\phi T \phi \otimes \phi^{i} \phi^{j}\right)=+\frac{2}{z^{i+j+2}} \quad \text { if } i=\text { odd, } j=\text { even. } \\
h(z) a_{n}^{\text {even }}= \\
\mathcal{E}_{z} h \cdot a_{n}^{\text {even }}+\frac{1}{2} e^{z D} \phi \cdot\left(\sum(-1)^{i} \phi \phi^{2} \phi^{4} \cdots \widehat{\phi^{2 i}} \cdots \phi^{2 n} r_{z, 0}\left(T \phi \otimes \phi^{2 i}\right)\right)- \\
\quad-\frac{1}{2} e^{-z D} \phi \cdot\left(\sum(-1)^{i} \phi \phi^{2} \phi^{4} \cdots \widehat{\phi^{2 i}} \cdots \phi^{2 n}\right) r_{z, 0}\left(\phi \otimes \phi^{2 i}\right)+ \\
+\sum_{i, j}(-1)^{2 i+2 j} 1 \cdot \phi \phi^{2} \phi^{4} \cdots \widehat{\phi^{2 i}} \ldots \widehat{\phi^{2 j}} \cdots \phi^{2 n} r_{z, 0}\left(\phi T \phi \otimes \phi^{2 i} \phi^{2 j}\right) .
\end{gathered}
$$

Hence

$$
h(z) a_{n}^{\text {even }}=\mathcal{E}_{z} h \cdot a_{n}^{\text {even }}-\frac{1}{2} \sum_{i}(-1)^{i}\left(e^{z D} \phi+e^{-z D} \phi\right) \cdot\left(\phi \phi^{2} \phi^{4} \cdots \widehat{\phi^{2 i}} \cdot \phi^{2 n} \frac{1}{z^{2 i+1}}\right) .
$$

Thus, there will be no nonzero contribution to any power of z less than -1 in (V.7), as we are multiplying by one of the $\phi^{2 l}$ that is already in the product $\phi \phi^{2} \phi^{4} \cdots \widehat{\phi^{2 i}} \cdot \phi^{2 n}$, and thus getting 0 . On the other hand, the contribution to the coefficient in front of $z^{-1}$ is $-2 n a_{n}^{\text {even }}$, as we get a $z^{-1}$ precisely when we are multiplying by the $\phi^{2 i} z^{2 i}$ term from $\frac{1}{2}\left(e^{z D} \phi+(-1)^{i} e^{-z D} \phi\right)$ which exactly complements the $\phi \phi^{2} \phi^{4} \cdots \widehat{\phi^{2 i}} \cdots \phi^{2 n}$. The minus sign in $-2 n a_{n}^{\text {even }}$ is due to the fact that when multiplying $(-1)^{i} \phi^{2 i} \cdot \phi \phi^{2} \phi^{4} \cdots \widehat{\phi^{2 i}} \cdots \phi^{2 n}$ we get $+a_{n}^{\text {even }}$. These

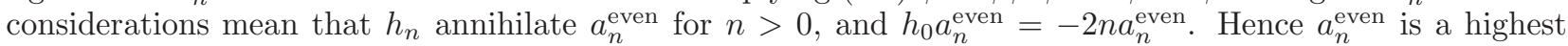
weight vector with weight $-2 n$.

Closer observation of the positive powers of $z$ in (V.7 shows which elements of $W=F_{D}$ can be generated from the highest weight vector $a_{n}^{\text {even }}$ : the elements with $n+2 m$ factors, $m \geq 0$, with exactly $m$ factors $\phi^{p}$ where $p$ is odd. We see that $W=F_{D}$ is bi-graded: first by the number $n$ of factors in an element $a=\phi^{k_{1}} \cdot \phi^{k_{2}} \cdots \phi^{k_{n}}$, $k_{1}<k_{2}<\cdots<k_{n}$, and second by the difference between how many of these $k_{i}$ are odd minus how many of them are even (we will call the second grading "derivative grading"). For example, the element $\phi D \phi$ is in the highest weight module generated by the highest vector $1=a_{0}^{\text {even }}$, as it has derivative grading 0 equal to the highest weight of 1 .

Now let $a_{n}^{\text {odd }}=\phi^{1} \phi^{3} \cdots \phi^{2 n-1}, a_{1}^{\text {odd }}=\phi^{1}=D \phi$. We claim $a_{n}^{\text {odd }}$ is a highest weight vector for $\mathcal{H}_{\mathbb{Z}}$. Similar calculations as for $a_{n}^{\text {even }}$ show that

$$
h(z) a_{n}^{\text {even }}=\mathcal{E}_{z} h \cdot a_{n}^{\text {odd }}+\frac{1}{2} \sum_{i}(-1)^{i}\left(e^{z D} \phi-e^{-z D} \phi\right) \cdot\left(\phi^{1} \phi^{3} \cdots \widehat{\phi^{2 i-1}} \cdots \phi^{2 n-1} \frac{1}{z^{2 i}}\right)
$$


Similar considerations as for $a_{n}^{\text {even }}$ hold for the $a_{n}^{\text {odd }}$, the difference is in the minus sign, i.e., $a_{n}^{\text {odd }}$ is a highest weight vector with highest weight $n$ for the Heisenberg algebra $\mathcal{H}_{\mathbb{Z}}$. We also see that any element with derivative grading $n \in \mathbb{Z}$ will be in the highest module with highest weight $n$.

From the above consideration it follows that the following decomposition of the space of states $F_{D}=W$ holds:

$$
W=F_{D} \cong \oplus_{i \in \mathbb{Z}} B_{i} .
$$

Any highest weight module for $\mathcal{H}_{\mathbb{Z}}$ with highest weight $k$ is isomorphic to $\mathbb{C}\left[x_{1}, x_{2}, \ldots, x_{n}, \ldots\right]$ via

$$
h_{n}=n \partial_{x_{n}} \quad \text { for } n>0 ; \quad h_{-n}=x_{n} . \quad \text { for } n>0 ; \quad h_{0}=k .
$$

Thus we can rewrite

$$
W=F_{D} \cong \oplus_{i \in \mathbb{Z}} B_{i} \cong \mathbb{C}\left[e_{\phi}^{\alpha}, e_{\phi}^{-\alpha}\right] \otimes \mathbb{C}\left[x_{1}, x_{2}, \ldots, x_{n}, \ldots\right]=B_{D},
$$

where $e_{\phi}^{n \alpha}, e_{\phi}^{-n \alpha}$ are for now just labels for the highest weight vectors, but as we will see in the next section the notation is used because it is coming from the bosonic side, which is a twisted vertex algebra based on a Leibnitz module over a rank one abelian group (see Example IV.10). Denote the right hand side of the isomorphism above by $B_{D}$, this is the bosonic space of states for the boson-fermion correspondence of type D-A.

We modify the labeling of the highest weight vectors, as formulas (III.16) and (III.17) of Proposition III.18 look simpler with such identification. Let

$$
\begin{aligned}
& e_{\phi}^{n \alpha} \cong \phi^{2 n-1} \cdot \phi^{2 n-3} \cdots \phi^{3} \cdot \phi^{1}=(-1)^{n-1} a_{n}^{\text {odd }} \quad n>0 \\
& e_{\phi}^{-n \alpha} \cong \phi^{2 n} \cdot \phi^{2 n-2} \cdots \phi^{2} \cdot \phi=(-1)^{n} a_{n}^{\text {even }} \quad n \geq 0
\end{aligned}
$$

To prove Proposition \II.15 we first calculate the OPE $h(z) \phi^{D}(w)$. We use CorollaryIV.31 as all the poles are simple here. The coefficients coming in front of the first order poles then are: for $\frac{1}{z-w}$ we get $-Y(T \phi \cdot 1, w)=$ $-Y(T \phi, w)$; for $\frac{1}{z+w}$ we get $-Y(T \phi \cdot 1, w)=-Y(T \phi, w)$. Thus

$$
\begin{aligned}
& h(z) \phi^{D}(w) \sim \frac{1}{2}\left(-\frac{Y(T \phi, w)}{z-w}-\frac{Y(T \phi, w)}{z+w}\right) \sim \frac{-z}{z^{2}-w^{2}}(T \phi)^{D}(w), \\
& h(z)(T \phi)^{D}(w) \sim \frac{1}{2}\left(-\frac{Y(\phi, w)}{z-w}-\frac{Y(\phi, w)}{z+w}\right) \sim \frac{-z}{z^{2}-w^{2}} \phi^{D}(w),
\end{aligned}
$$

and

$$
\begin{aligned}
h(z) \frac{1}{2}\left(\phi^{D}(w)+T \phi^{D}(w)\right) & \sim-\frac{z}{z^{2}-w^{2}} \frac{1}{2}\left(\phi^{D}(w)+T \phi^{D}(w)\right), \\
h(z) \frac{1}{2}\left(\phi^{D}(w)-T \phi^{D}(w)\right) & \sim \frac{z}{z^{2}-w^{2}} \frac{1}{2}\left(\phi^{D}(w)-T \phi^{D}(w)\right) .
\end{aligned}
$$

Denote $e_{\phi}^{-\alpha}(w)=\frac{1}{2}\left(\phi^{D}(w)+T \phi^{D}(w)\right), \quad e_{\phi}^{\alpha}(w)=\frac{1}{2}\left(\phi^{D}(w)-T \phi^{D}(w)\right)$, we have

$$
h(z) e_{\phi}^{-\alpha}(w) \sim-\frac{z}{z^{2}-w^{2}} e_{\phi}^{-\alpha}(w), \quad h(z) e_{\phi}^{\alpha}(w) \sim \frac{z}{z^{2}-w^{2}} e_{\phi}^{\alpha}(w) .
$$

These OPEs immediately imply the commutation relations

$$
\begin{aligned}
& {\left[h(z), e_{\phi}^{-\alpha}(w)\right]=-\left(i_{z, w}-i_{w, z}\right) \frac{z}{z^{2}-w^{2}} e_{\phi}^{-\alpha}(w)=-\frac{1}{2}(\delta(z-w)+\delta(z+w)) e_{\phi}^{-\alpha}(w),} \\
& {\left[h(z), e_{\phi}^{\alpha}(w)\right]=\left(i_{z, w}-i_{w, z}\right) \frac{z}{z^{2}-w^{2}} e_{\phi}^{\alpha}(w)=\frac{1}{2}(\delta(z-w)+\delta(z+w)) e_{\phi}^{\alpha}(w) .}
\end{aligned}
$$

From these commutation relations and the exact description of the split of $W=F_{D}$ into Heisenberg submodules, standard calculational lemmas (see for example [Kac90], [Wak01]) will give us

$$
\begin{aligned}
\frac{1}{2}\left(\phi^{D}(w)+\phi^{D}(-w)\right) & =e_{\phi}^{-\alpha}(z)=\exp \left(-\sum_{n \geq 1} \frac{h_{-n}}{n} z^{2 n}\right) \exp \left(\sum_{n \geq 1} \frac{h_{n}}{n} z^{-2 n}\right) e_{\phi}^{-\alpha} z^{-2 \partial_{\alpha}}, \\
\frac{1}{2}\left(\phi^{D}(w)-\phi^{D}(-w)\right) & =e_{\phi}^{\alpha}(z)=\exp \left(\sum_{n \geq 1} \frac{h_{-n}}{n} z^{2 n}\right) \exp \left(-\sum_{n \geq 1} \frac{h_{n}}{n} z^{-2 n}\right) e_{\phi}^{\alpha} z^{2 \partial_{\alpha}+1},
\end{aligned}
$$


where the operators $e_{\phi}^{\alpha}, e_{\phi}^{-\alpha}, z^{\partial_{\alpha}}$ and $z^{-\partial_{\alpha}}$ act in an obvious way on the space $F_{D} \cong B_{D}$ as in (V.9). This proves Proposition

Formulas (V.13) and (V.14) alone completely determine the twisted vertex algebra isomorphism between the two twisted vertex algebras: the fermionic with space of states $F_{D}$ and the bosonic with space of states $B_{D}$. Nevertheless, in the section $\mathrm{VG}$ we will present a bicharacter construction of the bosonic side of the boson-fermion correspondences.

Before moving to the bosonic sides, we want to give the bicharacter description of the fermionic side of the boson-fermion correspondence of type A. Even though it is a super vertex algebra (thus a twisted vertex algebra of order 1), the bicharacter construction gives us something new: a general formula for the vacuum expectation values that specializes to the determinant formula (II.16) in the case of the charged free fermions of type A.

\section{E. Twisted vertex algebras based on $\mathbb{C}\{\phi, \psi\}$ : determinant vacuum expectation values}

Recall that for the bicharacter construction examples are grouped based on the Hopf algebra $M$, i.e. one keeps $M$ the same, but changes the bicharacter. We dealt with two examples based on $M=\mathbb{C}\{\phi\}$. Now we want to work with $M=\mathbb{C}\{\phi, \psi\}$, as in example IV.13 A choice of a super symmetric bicharacter on $M=\mathbb{C}\{\phi, \psi\}$ is determined by the choice of three bicharacter values: $r_{z, w}(\phi \otimes \phi), r_{z, w}(\phi \otimes \psi)$ and $r_{z, w}(\psi \otimes \psi)\left(\right.$ as $r_{z, w}(\psi \otimes \phi)=-r_{w, z}(\phi \otimes \psi)$ from the super-symmetry). We will restrict ourselves with the case when $r_{z, w}(\phi \otimes \phi)=r_{z, w}(\psi \otimes \psi)=0$, thus we are only choosing $r_{z, w}(\phi \otimes \psi)$. The pair $(\mathbb{C}\{\phi, \psi\}, r)$ for any covariant bicharacter will generate a twisted vertex algebra with space of fields $V=H_{T_{\epsilon}}^{N}(\mathbb{C}\{\phi, \psi\})$, and space of states $W=H_{D}(\mathbb{C}\{\phi, \psi\})$ as in theorem IV.41.

We want to derive a formula for the vacuum expectation values in any twisted vertex algebra based on the pair $(\mathbb{C}\{\phi, \psi\}, r)$, with the bicharacter $r$ chosen as above. Recall $\langle\mid\rangle: W \otimes W \rightarrow \mathbb{C}$ is a symmetric bilinear form on the space of states $W$, such that the vacuum vector $1=|0\rangle$ is orthogonal to all other generators of the Hopf algebra $W$ and also has norm 1.

Proposition V.4 Let $V$ be a twisted vertex algebra based on $M=\mathbb{C}\{\phi, \psi\}$ and a supersymmetric bicharacter $r$ (i.e., $V=H_{D}(\mathbb{C}\{\phi, T \phi, \psi, T \psi\})$ and $W=H_{D}(\mathbb{C}\{\phi, \psi\})$. Denote by $\phi(z)$ and $\psi(z)$ the fields $Y(\phi, z)$ and $Y(\psi, z)$ produced by IV.22, via (IV.21). The following formula for the vacuum expectation values holds:

$$
\left\langle 0\left|\phi\left(z_{1}\right) \phi\left(z_{2}\right) \ldots \phi\left(z_{n}\right) \psi\left(w_{1}\right) \psi\left(w_{2}\right) \ldots \psi\left(w_{n}\right)\right| 0\right\rangle=(-1)^{n(n-1) / 2} i_{z, w} \operatorname{det}\left(r_{z_{i}, w_{j}}(\phi \otimes \psi)\right)_{i, j=1}^{n} .
$$

Here as usual det denotes the determinant of an $n$ by $n$ square matrix and $i_{z ; w}$ stands for the expansion $i_{z_{1}, z_{2}, \ldots, z_{n}, w_{1}, w_{2}, \ldots, w_{n}}$.

Proof: To calculate the vacuum expectation values we calculate instead the vacuum expectation values of the analytic continuation $X_{z_{1}, z_{2}, \ldots, z_{n}, w_{1}, w_{2}, \ldots, w_{n}}(\phi \otimes \phi \ldots \phi \otimes \psi \otimes \psi \cdots \psi)$. We will use Lemma IV.39 which gives us a formula for the analytic continuation in terms of the bicharacter. Both $\phi$ and $\psi$ are primitive elements, we have

$$
\begin{aligned}
& \triangle^{2 n}(\phi)=\phi \otimes 1 \otimes \ldots \otimes 1+1 \otimes \phi \otimes \ldots \otimes 1+1 \otimes 1 \otimes \phi \otimes \ldots \otimes 1+\ldots 1 \otimes 1 \otimes \ldots \otimes \phi, \\
& \triangle^{2 n}(\psi)=\psi \otimes 1 \otimes \ldots \otimes 1+1 \otimes \psi \otimes \ldots \otimes 1+1 \otimes 1 \otimes \psi \otimes \ldots \otimes 1+\ldots 1 \otimes 1 \otimes \ldots \otimes \psi
\end{aligned}
$$

We need three observations:

1. Since for the bilinear form the vacuum vector $1=|0\rangle$ spans an orthogonal subspace on its own (and in particular is orthogonal to $\phi$ and $\psi$ and their descendants), the only contributions to the vacuum expectation values will come from the terms in the multivariable field where the coproducts have 1 as a first term; the other terms will not contribute. That forces us to work with the $(2 n)$-character

$r_{z_{1}, z_{2}, \ldots, z_{n}, w_{1}, w_{2}, \ldots, w_{n}}(\phi \otimes \phi \ldots \phi \otimes \psi \otimes \psi \cdots \psi)$.

2. To continue the previous observation, no sign contribution will come from the first $(-1)$ factor in the $\operatorname{sign}\left(M_{\Delta^{2 n-1}}^{\vec{k}}(\phi, \ldots, \phi, \psi, \cdots, \psi)\right)$ as the only contributing matrices are those with the first columns consisting entirely of $1 \mathrm{~s}$ (as 1 is even).

3. Since $\phi$ and $\psi$ are primitive elements we have $r_{z, w}(\phi \otimes 1)=r_{z, w}(1 \otimes \phi)=0$ and $r_{z, w}(\psi \otimes 1)=r_{z, w}(1 \otimes \psi)=$ 0 for any bicharacter. Thus the only contributions in the $(2 n)$-character $r_{z_{1}, z_{2}, \ldots, z_{n}, w_{1}, w_{2}, \ldots, w_{n}}(\phi \otimes \phi \ldots \phi \otimes$ $\psi \otimes \psi \cdots \psi$ ) will come from the following situation: a nonzero summand in this $(2 n)$-character will be 
a product of nonzero bicharacter factors, and that happens when in the "permuted order" (see remark IV.40) we have a sequence of pairs: $(1,1)$ pairs (trivial, as $\left.r_{z, w}(1 \otimes 1)=1\right)$ or $(\phi, \psi)$ pairs (nontrivial). If there is a mixed pair $(1, \phi)$ or $(\phi, 1)$ as a factor in a summand, that summand will be 0 . Note also that that the definition of a $(2 n)$-character doesn't allow for pairs $(\psi, \phi)$, as it uses the "permuted order" in its definition. So a nonzero summand will have exactly $n$ such nontrivial contributing pairs $(\phi, \psi)$, and each pair forms one bicharacter $r_{z_{i}, w_{j}}(\phi \otimes \psi)$. Which means that a nonzero summand will consist of the product $\prod_{k=1}^{n} r_{z_{k}, w_{j_{k}}}(\phi \otimes \psi)$ times a sign factor, where $j_{1}, j_{2}, \ldots, j_{n}$ is a permutation of $1,2, \ldots, n$.

Thus, we have

$$
\begin{aligned}
& X_{z_{1}, z_{2}, \ldots, z_{n}, w_{1}, w_{2}, \ldots, w_{n}}(\phi \otimes \phi \ldots \phi \otimes \psi \otimes \psi \cdots \psi) \\
& =\sum_{\text {coproducts }} \operatorname{sign}\left(M_{\Delta^{2 n-1}}^{\vec{k}}(\phi, \ldots, \phi, \psi, \ldots, \psi)\right) \mathcal{E}_{z_{1}} \phi^{\prime} \ldots \mathcal{E}_{z_{n}} \phi^{\prime} \mathcal{E}_{w_{1}} \psi^{\prime} \ldots \mathcal{E}_{w_{n}} \psi^{\prime} . \\
& \quad \cdot r_{z_{1}, z_{2}, \ldots, z_{n}, w_{1}, w_{2}, \ldots, w_{n}}\left(\phi^{\prime \prime} \otimes \ldots \phi^{\prime \prime} \otimes \psi^{\prime \prime} \ldots \otimes \psi^{\prime \prime}\right) \\
& =\sum_{\substack{\text { contr. } \\
\text { coproducts }}} \operatorname{sign}\left(M_{\Delta^{2 n-1}}^{\vec{k}}(\phi, \ldots, \phi, \psi, \ldots, \psi)\right) 1 . \\
& =(-1)^{n(n-1) / 2} \sum_{P} \epsilon(P) 1 \cdot r_{z_{1}, w_{j_{1}}}(\phi \otimes \psi) r_{z_{2}, w_{j_{2}}}(\phi \otimes \psi) \cdots r_{z_{n}, w_{j_{n}}}(\phi \otimes \psi)+\text { other terms. }
\end{aligned}
$$

The sum is over all permutations $j_{1}, j_{2}, \ldots, j_{n}$ of $1,2, \ldots, n$. The sign contribution from any contributing matrix $\left.M_{\Delta^{2 n-1}}^{\vec{k}}(\phi, \ldots, \phi, \psi, \ldots, \psi)\right)$ consist of two factors: the first factor is the sign due to all the " $w$ 's passing through the " $z$ 's", which factor is exactly $(-1)^{n(n-1) / 2}$. The second factor is precisely the sign of the corresponding permutation, since $\phi$ and $\psi$ are odd (see remark IV.40 and observation 3 above, also permuting a pair across a pair contributes no minus sign). That produces precisely the determinant $(-1)^{n(n-1) / 2} \operatorname{det}\left(r_{z_{i}, w_{j}}(\phi \otimes \psi)\right)_{i, j=1}^{n}$.

\section{F. Super vertex algebra based on $\mathbb{C}\{\phi, \psi\}$ : the charged free fermions of type A}

We start with the fermionic side of the boson-fermion correspondence of type A, even though it is a super vertex algebra. We would like to recall that a super vertex algebra is in fact a twisted vertex algebra of order $N=1$. The bicharacter description of the fermionic side of the correspondence was given in [Ang08], we will recall it briefly for completeness, also the formula for the vacuum expectation values is new to this paper. We choose $V=H_{D}(\mathbb{C}\{\phi, \psi\}), W=V=H_{D}(\mathbb{C}\{\phi, \psi\})$, i.e., $\pi=I d_{W}$, and $r_{z, w}$ be the $H_{D} \otimes H_{D^{-} \text {covariant }}$ bicharacter $r^{A_{f}}$ on $V=W$ generated by

$$
r_{z, w}(\phi \otimes \psi)=\frac{1}{z-w}, \quad r_{z, w}(\phi \otimes \phi)=r_{z, w}(\phi \otimes \psi)=0,
$$

which produces a super-symmetric shift restricted bicharacter as in section VE Let $Y$ be the field-state correspondence defined by (IV.22), via (IV.21). The set of data $\left(V, W, \pi=I d_{W}, Y\right)$ constructed as above satisfies the definition of a twisted vertex algebra, which is in fact a super vertex algebra, as the bicharacter has poles only at $z=w$. Which vertex algebra it is is determined once we write out the OPEs for the Heisenberg relations. We can define a Heisenberg element $h=\phi \cdot \psi$, which leads to $h(z)=: \phi(z) \psi(z)$ immediately from lemma IV.33. We have from theorem IV.29 that

$$
h(z) h(w) \sim \frac{1}{(z-w)^{2}} \quad \text { and } \quad h(z) \phi(w) \sim \frac{1}{z-w} \phi(w),
$$

which in commutation relations is:

$$
[h(z), \phi(w)]=\left(i_{z, w}-i_{w, z}\right) \frac{1}{z-w} \cdot \phi(w)=\delta(z-w) \phi(w) .
$$

From the last two equations it is a standard calculation that formulas (II.22) and (II.23) follow (these calculations are done for example in [Kac98] specifically for the boson-fermion correspondence of type A, as well as in Wak01]). 
Thus we have shown that the pair $\left(\mathbb{C}\{\phi, \psi\}, r_{z, w}(\phi \otimes \psi)=\frac{1}{z-w}\right)$ generates and describes the super vertex algebra of the charged free fermions, which is the fermionic side of the boson-fermion correspondence of type A.

We can directly see that the determinant formula for the vacuum expectation values (II.16) is a special case of Proposition V.4.

We won't give more examples here of a twisted vertex algebra based on $\mathbb{C}\{\phi, \psi\}$, but as was shown above such examples are easy to produce, as one just chooses a different bicharacter value $r_{z, w}(\phi \otimes \psi)$. Instead we move to the twisted vertex algebras that constitute the bosonic sides of the boson-fermion correspondences.

\section{G. Twisted vertex algebras based on $\mathbb{C}[\mathbb{Z} \alpha]$ and a choice of a bicharacter}

In this section we fix $M$ to be the Hopf algebra $L_{1}=\mathbb{C}[\mathbb{Z} \alpha]$, the group algebra of the rank-one free abelian group $\mathbb{Z} \alpha$, as in Example IV.10. We constructed the free Leibnitz module $\tilde{V}=H_{T_{\epsilon}}^{N}\left(L_{1}\right)$, and its sub-Hopf algebra $\tilde{W}=H_{D}\left(L_{1}\right)$. If we want to define a $H_{T_{\epsilon}}^{N} \otimes H_{T_{\epsilon}}^{N}$-covariant bicharacter on $H_{T_{\epsilon}}^{N}(M)$ it is clear that we can only choose one bicharacter value, that of $r_{z, w}\left(e^{\alpha} \otimes e^{\alpha}\right)$. Recall (Example IV.10) that we defined an element $h=\left(D e^{\alpha}\right) e^{-\alpha}$, which is primitive; note that $h \in \tilde{W} \subset \tilde{V}$.

Lemma V.5 The following hold for any covariant bicharacter on $\tilde{V}$ :

$$
\begin{aligned}
& r_{z, w}\left(h \otimes e^{m \alpha}\right)=m \partial_{z} \log r_{z, w}\left(e^{\alpha} \otimes e^{\alpha}\right) \\
& r_{z, w}(h \otimes h)=\partial_{z} \partial_{w} \log r_{z, w}\left(e^{\alpha} \otimes e^{\alpha}\right) ; \\
& h(z) h(w) \sim I d_{W} \cdot r_{z, w}(h \otimes h) ; \\
& h(z) e^{m \alpha}(w) \sim e^{m \alpha}(w) \cdot r_{z, w}\left(h \otimes e^{m \alpha}\right) .
\end{aligned}
$$

\section{H. Twisted vertex algebras based on $\mathbb{C}[\mathbb{Z} \alpha]$ : product vacuum expectation values}

Let $V$ be a twisted vertex algebra based on $L_{1}=\mathbb{C}[\mathbb{Z} \alpha]$ and a supersymmetric bicharacter $r$ (by use of Theorem IV.41) with space of states $W=H_{D}\left(L_{1}\right)$. Denote by $e^{m \alpha}(z)$ the field $Y\left(e^{m \alpha}, z\right)$ produced by definition IV.22 via (IV.21). Let the projection map $\pi$ from the space of fields to the space of states satisfy $\pi\left(e^{m \alpha}\right) \neq 0$ for any $m \in \mathbb{Z}$ (which holds for $\pi=\pi_{T}$ ).

Proposition V.6 The following formula for the vacuum expectation values holds:

$$
\left\langle 0\left|e^{m_{1} \alpha}\left(z_{1}\right) e^{m_{2} \alpha}\left(z_{2}\right) \ldots e^{m_{n} \alpha}\left(z_{n}\right)\right| 0\right\rangle=i_{z} \delta_{m_{1}+m_{2}+\cdots+m_{n}, 0} \prod_{i<j=1}^{n} r_{z_{i}, z_{j}}\left(e^{m_{i} \alpha} \otimes e^{m_{j} \alpha}\right) .
$$

Here $i_{z}$ stands for the expansion $i_{z_{1}, z_{1}, \ldots, z_{n}}$.

Proof: From Lemma IV.39, the fact that the parity is entirely even, and the elements $e^{m_{k} \alpha}$ are grouplike we have

$X_{z_{1}, z_{2}, \ldots, z_{n}}\left(e^{m_{1} \alpha} \otimes e^{m_{2} \alpha} \otimes \ldots \otimes e^{m_{n} \alpha}\right)=\mathcal{E}_{z_{1}} e^{m_{1} \alpha} \cdot \mathcal{E}_{z_{2}} e^{m_{2} \alpha} \ldots \mathcal{E}_{z_{n}} e^{m_{n} \alpha} \cdot r_{z_{1}, z_{2}, \ldots, z_{n}}\left(e^{m_{1} \alpha} \otimes e^{m_{2} \alpha} \otimes \ldots \otimes e^{m_{n} \alpha}\right)$.

We have

$$
\left\langle 0 \mid \mathcal{E}_{z_{1}} e^{m_{1} \alpha} \cdot \mathcal{E}_{z_{2}} e^{m_{2} \alpha} \cdots \mathcal{E}_{z_{n}} e^{m_{n} \alpha}\right\rangle=\left\langle 0 \mid \pi_{T}\left(e^{\left(m_{1}+m_{2}+\cdots+m_{n}\right) \alpha}\right)+O(z)\right\rangle .
$$

Since we required that the bilinear form is such that the vacuum vector $|0\rangle$ is orthogonal to all $e^{m \alpha}$, except for the $m=0$, then

$$
\left\langle 0 \mid e^{\left(m_{1}+m_{2}+\cdots+m_{n}\right) \alpha}\right\rangle=\delta_{m_{1}+m_{2}+\cdots+m_{n}, 0} .
$$

Note also that the $O(z)$ terms contain non-vacuum descendants of the $e^{m_{k} \alpha}$, and so do not contribute to the vacuum expectation value. Thus

$$
\left\langle 0 \mid X_{z_{1}, \ldots, z_{n}}\left(e^{m_{1} \alpha} \otimes \ldots \otimes e^{m_{n} \alpha}\right)\right\rangle=\delta_{m_{1}+\cdots+m_{n}, 0} r_{z_{1}, z_{2}, \ldots, z_{n}}\left(e^{m_{1} \alpha} \otimes e^{m_{2} \alpha} \otimes \ldots \otimes e^{m_{n} \alpha}\right) .
$$

Now since the elements $e^{m_{k} \alpha}$ are grouplike, the $n$-character $r_{z_{1}, z_{2}, \ldots, z_{n}}\left(e^{m_{1} \alpha} \otimes \ldots \otimes e^{m_{n} \alpha}\right)$ also has especially simple form:

$$
r_{z_{1}, z_{2}, \ldots, z_{n}}\left(e^{m_{1} \alpha} \otimes e^{m_{2} \alpha} \otimes \ldots \otimes e^{m_{n} \alpha}\right)=\prod_{i<j=1}^{n} r_{z_{i}, z_{j}}\left(e^{m_{i} \alpha} \otimes e^{m_{j} \alpha}\right)
$$




\section{Super vertex algebra based on $\mathbb{C}[\mathbb{Z} \alpha]$ : the free boson of type $A$}

We start with the bosonic side of the boson-fermion correspondence of type A, even though it is a super vertex algebra. We would like to recall that a super vertex algebra is in fact a twisted vertex algebra of order $N=1$. This section gives the bicharacter description of the bosonic side of the correspondence.

We choose $V=H_{D}\left(L_{1}\right), W=V=H_{D}\left(L_{1}\right)$, i.e., $\pi=I d_{W}$, and $r_{z, w}^{A_{b}}$ be the $H_{D} \otimes H_{D}$-covariant bicharacter on $V=W$ generated by

$$
r_{z, w}\left(e^{\alpha} \otimes e^{\alpha}\right)=z-w,
$$

which is a super-symmetric shift restricted bicharacter. As was derived in the previous section for general bicharacter

$$
r_{z, w}\left(h \otimes e^{m \alpha}\right)=m \frac{1}{z-w}, \quad r_{z, w}(h \otimes h)=\frac{1}{(z-w)^{2}} .
$$

Let $Y$ be the field-state correspondence defined by (IV.22), via (IV.21). The set of data $\left(V, W, \pi=I d_{W}, Y\right)$ constructed as above satisfies the definition of a twisted vertex algebra, which is in fact a super vertex algebra, as the bicharacter has poles only at $z=w$. Which particular vertex algebra we get is determined once we write the OPEs for the Heisenberg relation (V.21) and the exponential relation (V.22) as commutation relations:

$$
[h(z), h(w)]=\left(i_{z, w}-i_{w, z}\right) \frac{1}{(z-w)^{2}}=\partial_{w} \delta(z-w),
$$

which is precisely (II.18); and

$$
\left[h(z), e^{m \alpha}(w)\right]=\left(i_{z, w}-i_{w, z}\right) \frac{m}{z-w} \cdot e^{m \alpha}(w)=m \delta(z-w) e^{m \alpha}(w) .
$$

From the last two equations it is a standard calculation that formulas (II.22) and (II.23) follow (these calculations are done for example in [Kac98] specifically for the boson-fermion correspondence of type A, as well as in Wak01]).

Thus we have shown that the pair $\left(L_{1}, r_{z, w}\left(e^{\alpha} \otimes e^{\alpha}\right)=z-w\right)$ generates and describes the super vertex algebra of the rank one odd lattice, which is the bosonic side of the boson-fermion correspondence of type A. To summarize all these considerations:

Theorem V.7 The boson-fermion correspondence of type $A$ is the isomorphism between two super vertex algebras: the fermionic side, which is the vertex algebra based on the pair $\left(\mathbb{C}\{\phi, \psi\}, r^{A_{f}}\right)$; and the bosonic side, which is the vertex algebra based on the pair $\left(\mathbb{C}[\mathbb{Z} \alpha], r^{A_{b}}\right)$.

We can directly see that (II.24) is a special case of Proposition V.6. as

$$
r_{z_{i}, w_{j}}\left(e^{\alpha} \otimes e^{-\alpha}\right)=\frac{1}{z_{i}-w_{j}}, \quad r_{z_{i}, z_{j}}\left(e^{\alpha} \otimes e^{\alpha}\right)=z_{i}-w_{j} \quad r_{w_{i}, w_{j}}\left(e^{-\alpha} \otimes e^{-\alpha}\right)=w_{i}-w_{j},
$$

thus

$$
\left\langle 0\left|e^{\alpha}\left(z_{1}\right) e^{\alpha}\left(z_{2}\right) \ldots e^{\alpha}\left(z_{n}\right) e^{-\alpha}\left(w_{1}\right) e^{-\alpha}\left(w_{2}\right) \ldots e^{-\alpha}\left(w_{n}\right)\right| 0\right\rangle=i_{z, w} \frac{\prod_{i<j}^{n}\left(\left(z_{i}-z_{j}\right)\left(w_{i}-w_{j}\right)\right)}{\prod_{i, j=1}^{n}\left(z_{i}-w_{j}\right)} .
$$

Here $i_{z ; w}$ stands for the expansion $i_{z_{1}, z_{2}, \ldots, z_{n}, w_{1}, \ldots, w_{n}}$. We see that since the boson-fermion correspondence identifies the fields $e^{\alpha}(z)=\phi^{B}(z)$, then Corollary $\amalg .15$ follows directly.

\section{J. Twisted vertex algebra based on $\mathbb{C}[\mathbb{Z} \alpha]$ : the free boson of type B}

We continue with the bosonic side of the boson-fermion correspondence of type B. This is the first example where the spaces of states and fields are not free Leibnitz modules, but quotients of a free Leibnitz module.

Recall the free Leibnitz module $\tilde{V}=H_{T_{\epsilon}}^{N}\left(L_{1}\right)$, and its sub-Hopf algebra $\tilde{W}=H_{D}\left(L_{1}\right)$. We again take $\epsilon=-1$ and write just $T$ instead of $T_{\epsilon}$. Let

$$
V=\tilde{V} /\left\{T e^{\alpha}=e^{-\alpha}\right\} .
$$


Denote the quotient relations generated from $\left\{T e^{\alpha}=e^{-\alpha}\right\}$ by $\mathcal{R}_{\mathcal{B}}$.

In order to define a $H_{T_{\epsilon}}^{2} \otimes H_{T}^{2}$-covariant bicharacter on $V$ we can only choose the bicharacter value $r_{z, w}\left(e^{\alpha} \otimes\right.$ $\left.e^{\alpha}\right)$. Further, in order to be able to restrict this bicharacter to a bicharacter on $V=\tilde{V} / \mathcal{R}_{\mathcal{B}}$, it needs to be consistent with the relations $\mathcal{R}_{\mathcal{B}}$, thus

$$
r_{-z, w}\left(e^{\alpha} \otimes e^{\alpha}\right)=r_{z,-w}\left(e^{\alpha} \otimes e^{\alpha}\right)=\frac{1}{r_{z, w}\left(e^{\alpha} \otimes e^{\alpha}\right)} .
$$

Hence, we can choose

$$
r_{z, w}\left(e^{\alpha} \otimes e^{\alpha}\right)=\frac{z-w}{z+w}
$$

and this bicharacter value will generate a bicharacter $r^{B_{b}}$ on $V$ by covariance.

Now we turn to the exact description of the space of fields $V$ and the space of states $W$ of the twisted vertex algebra generated by the pair $\left(L_{1} / \mathcal{R}_{\mathcal{B}}, r^{B_{b}}\right)$. From Example IV.10 the free Leibnitz module $H_{T}^{2}\left(L_{1}\right)$ is isomorphic to $L_{2} \otimes H_{T}^{2}(\mathbb{C}[h])$, where $L_{2}$ is the group algebra $L_{2}=\mathbb{C}\left[\mathbb{Z} \alpha, \mathbb{Z} \alpha_{1}\right]$ of the free abelian group of rank 2 (we identify $T e^{\alpha}$, which is grouplike, with $\left.e^{\alpha_{1}}\right)$. Denote by $h_{\alpha}^{B}$ the element $h_{\alpha}^{B}=\frac{1}{2}\left(D e^{\alpha}\right) T e^{\alpha} \in V$, which coincides with $\frac{1}{2}\left(D e^{\alpha}\right) e^{-\alpha} \in V$ under the relation $\mathcal{R}_{\mathcal{B}}$. It follows then that $T D e^{\alpha}=-D T e^{\alpha}=-D e^{-\alpha}$. Therefore

$$
T h_{\alpha}^{B}=h_{\alpha}^{B}
$$

Thus under the imposed relations $\mathcal{R}_{\mathcal{B}}$ in $V$ we have $H_{T}^{2}(\mathbb{C}[h]) / \mathcal{R}_{\mathcal{B}}=H_{D}\left(\mathbb{C}\left[h_{\alpha}^{B}\right]\right)$ and

$$
V=L_{1} \otimes H_{D}\left(\mathbb{C}\left[h_{\alpha}^{B}\right]\right)
$$

The space of states $W$ is defined via the projection map $\pi: V \rightarrow W$, and in order to apply Theorem IV.41 we use as projection map the map from definition IV.15 adapted to the relations $\mathcal{R}_{\mathcal{B}}$. More precisely, define $\pi: V \rightarrow W$ to be the linear map defined by

$$
\pi\left(H_{T}^{2}\left(\mathbb{C}\left[h_{\alpha}^{B}\right]\right) / \mathcal{R}_{\mathcal{B}}\right)=I d, \quad \pi\left(T e^{n \alpha}\right)=e^{n \alpha}, \quad \pi\left(e^{n \alpha}\right)=e^{n \alpha}, n \in \mathbb{Z} .
$$

Denote by $\bar{v}$ the element of $W$ that is the projection of the element $v \in V$. We have

$$
\overline{1}=\overline{e^{\alpha}} \overline{e^{-\alpha}}=\pi\left(e^{\alpha} e^{-\alpha}\right)=\pi\left(e^{\alpha} T e^{\alpha}\right)=\pi\left(e^{\alpha}\right) \pi\left(T e^{\alpha}\right)=\pi\left(e^{2 \alpha}\right)=\overline{e^{2 \alpha}},
$$

thus we have in $W$

$$
\overline{e^{2 \alpha}}=1, \quad \overline{e^{\alpha}}=\overline{e^{-\alpha}}
$$

Thus $W=H_{D}\left(\mathbb{C}\left[h_{\alpha}^{B}\right]\right) \oplus e^{\alpha} H_{D}\left(\mathbb{C}\left[h_{\alpha}^{B}\right]\right)$ and as expected, $W=B_{B}$ as in Lemma III.11. Moreover, from Lemma $\mathrm{V.5} h_{\alpha}^{B}$ is a Heisenberg element: we use (V.21), which in this case specializes from (V.20) to

$$
h_{\alpha}^{B}(z) h_{\alpha}^{B}(w) \sim 1 \cdot r_{z, w}\left(h_{\alpha}^{B} \otimes h_{\alpha}^{B}\right) \sim 1 \cdot \frac{1}{4} \partial_{w} \partial_{z} \log \frac{z-w}{z+w} \sim 1 \cdot \frac{z^{2}+w^{2}}{2\left(z^{2}-w^{2}\right)^{2}} .
$$

Now the unexpected twist here is that $T h_{\alpha}^{B}=h_{\alpha}^{B}$, hence the field $h_{\alpha}^{B}(z)$ has only even powers of $z$, and we can write $h_{\alpha}^{B}(z)=\sum_{n \in \mathbb{Z}} h_{2 n+1} z^{-2 n}$. This immediately leads to the required commutation relations

$$
\left[h_{m}, h_{n}\right]=\frac{m}{2} \delta_{m+n, 0}, \quad m, n \text { odd integers. }
$$

Note that we can reindex this field $h_{\alpha}^{B}(z)$ in the following form: $h_{\alpha}^{B}(z)=\sum_{n \in \mathbb{Z}+1 / 2} \tilde{h}_{(n)} z^{-2 n-1}\left(\right.$ i.e., $h_{2 n}=\tilde{h}_{(n)}$ for $n \in \mathbb{Z}+1 / 2$ ), which translates to

$$
\left[\tilde{h}_{(m)}, \tilde{h}_{(n)}\right]=m \delta_{m+n, 0}, \quad m, n \in \mathbb{Z}+1 / 2
$$

and explains the name $\mathcal{H}_{\mathbb{Z}+1 / 2}$ for this Heisenberg algebra. 
Remark V.8 Note that

$$
h_{\alpha}^{B}(z)=z \cdot h_{\phi}^{B}(z),
$$

which is allowed in an isomorphism of twisted vertex algebras: the doubly infinite sequences of the modes of the two fields $h_{\alpha}^{B}(z)$ and $h^{B}(z)$ are identical, except for the shift in the indexing (the multiplication by $z$ ).

For the OPEs of the fields $e^{m \alpha}(w)$ with $h_{\alpha}^{B}(z)$, from (V.22) we get

$$
h_{\alpha}^{B}(z) e^{m \alpha}(w) \sim m e^{m \alpha}(w) \cdot \frac{w}{z^{2}-w^{2}} .
$$

We see that if we identify $e^{\alpha}(z)=\phi^{B}(z)$, this OPE implies the exponential operator formula (III.10) of the boson-fermion correspondence.

Thus we have shown that the pair $\left(L_{1} / \mathcal{R}_{\mathcal{B}}, r^{B_{b}}\right)$ generates the twisted vertex algebra which constitutes the bosonic side of the boson-fermion correspondence of type $\mathrm{B}$, and we have

Theorem V.9 The boson-fermion correspondence of type $B$ is an isomorphism between two twisted vertex algebras: the fermionic side, which is the vertex algebra based on the pair $\left(\mathbb{C}\{\phi\}, r^{B_{f}}\right)$; and the bosonic side, which is the twisted vertex algebra based on the pair $\left(\mathbb{C}[\mathbb{Z} \alpha] / \mathcal{R}_{\mathcal{B}}, r^{B_{b}}\right)$.

Lemma 111.15 follows directly: Since the boson-fermion correspondence identifies the fields $e^{\alpha}(z)=\phi^{B}(z)$, we can directly calculate the vacuum expectation values on each side of the correspondence of type $B$ and equate. As a special case of Proposition V.6 we have for the bosonic side:

$$
\left\langle 0\left|e^{\alpha}\left(z_{1}\right) e^{\alpha}\left(z_{2}\right) \ldots e^{\alpha}\left(z_{2 n}\right)\right| 0\right\rangle=i_{z} \prod_{i<j}^{2 n} \frac{z_{i}-z_{j}}{z_{i}+z_{j}} .
$$

Similarly, the vacuum expectation values on the fermionic side follow directly from Proposition $\nabla .1$.

\section{K. Twisted vertex algebra based on $\mathbb{C}[\mathbb{Z} \alpha]$ : the free boson of type D-A}

We continue with the bosonic side of the boson-fermion correspondence of type D-A.

We are again working with the free Leibnitz module $\tilde{V}=H_{T_{\epsilon}}^{N}\left(L_{1}\right)$, and its sub-Hopf algebra $\tilde{W}=H_{D}\left(L_{1}\right)$, $T=T_{\epsilon}$. For the bosonic space of type D-A we let

$$
V=\tilde{V} /\left\{T e^{\alpha}=e^{\alpha}\right\},
$$

i.e., $V$ is the quotient Leibnitz module modulo the relations $\mathcal{R}_{\mathcal{D}}$ generated by $\left\{T e^{\alpha}=e^{\alpha}\right\}$.

As we did in the previous section, if we want to define a $H_{T}^{2} \otimes H_{T}^{2}$-covariant bicharacter on $V$, we need to choose a bicharacter value $r_{z, w}\left(e^{\alpha} \otimes e^{\alpha}\right)$ which is consistent with the relations $\mathcal{R}_{\mathcal{D}}$. It follows that $r_{z, w}\left(e^{\alpha} \otimes e^{\alpha}\right)$ needs to be even as a function of both $z$ and $w$, as well as supersymmetric with exchange of $z$ and $w$. Thus we can choose

$$
r_{z, w}\left(e^{\alpha} \otimes e^{\alpha}\right)=z^{2}-w^{2},
$$

which will generate a bicharacter $r^{D_{b}}$ on $V$ by covariance.

Now we turn to the exact description of the space of fields $V$ and the space of states $W$ of this twisted vertex algebra. Denote by $h_{\alpha}^{D}$ the element $\frac{1}{2}\left(D e^{\alpha}\right) e^{-\alpha} \in V$, which we know is a Heisenberg element. In $V$ due to the relations $\mathcal{R}_{\mathcal{D}}$ we have $T D e^{\alpha}=-D T e^{\alpha}=-D e^{\alpha}$, and $T e^{-\alpha}=e^{-\alpha}$. Thus

$$
T h_{\alpha}^{D}=\frac{1}{2}\left(T D e^{\alpha}\right) T e^{-\alpha}=-\frac{1}{2} D T e^{\alpha} e^{-\alpha}=-\frac{1}{2} D e^{\alpha} e^{-\alpha}=-h_{\alpha}^{D} .
$$

Hence

$$
T h_{\alpha}^{D}=-h_{\alpha}^{D},
$$

thus under the imposed relations $\mathcal{R}_{\mathcal{D}}$ in $V$ we again have $H_{T}^{2}(\mathbb{C}[h]) / \mathcal{R}_{\mathcal{D}}=H_{D}\left(\mathbb{C}\left[h_{\alpha}^{D}\right]\right)$, although now $h_{\alpha}^{D}$ is odd under $T$. Hence

$$
V=L_{1} \otimes H_{D}\left(\mathbb{C}\left[h_{\alpha}^{D}\right]\right) .
$$


We define the space of states $W$ to be equal to $V$, i.e., we take as projection map $\pi: V \rightarrow W$ the identity map on $V$, which is consistent with the projection map $\pi_{T}$ modulo $\mathcal{R}_{\mathcal{D}}$. Hence we can again use Theorem IV.41 to get a twisted vertex algebra.

From (V.21) and (V.20) we have

$$
h_{\alpha}^{D}(z) h_{\alpha}^{D}(w) \sim 1 \cdot \frac{z w}{\left(z^{2}-w^{2}\right)^{2}} .
$$

Here $T h_{\alpha}^{D}=-h_{\alpha}^{D}$, hence the field $h_{\alpha}^{D}(z)$ has only odd powers of $z$, and we can write it as $h_{\alpha}^{D}(z)=$ $\sum_{n \in \mathbb{Z}} h_{n} z^{-2 n-1}$. Note that the field $h_{\alpha}^{D}(z)$ has the same OPE as the Heisenberg field $h^{D}(z)$, (III.13), as required.

On the bosonic side it is easy to identify the split into irreducible Heisenberg modules, as the highest weight vectors are precisely the elements $e^{n \alpha} \in V \equiv W, n \in \mathbb{Z}$. Hence as Heisenberg modules

$$
\begin{aligned}
V \equiv W & \equiv \mathbb{C}\left[e^{\alpha}, e^{-\alpha}\right] \otimes \mathbb{C}\left[h_{\alpha}^{D}, D h_{\alpha}^{D}, \ldots, D^{(n)} h_{\alpha}^{D}, \ldots\right] \cong \\
& \cong \mathbb{C}\left[e^{\alpha}, e^{-\alpha}\right] \otimes \mathbb{C}\left[x_{1}, x_{2}, \ldots, x_{n}, \ldots\right] \cong \oplus_{i \in \mathbb{Z}} B_{i}=B_{D}
\end{aligned}
$$

Now it remains to calculate the OPEs of the fields $e^{m \alpha}(w)$ with $h_{\alpha}^{D}(z)$, from (V.22) we get

$$
h_{\alpha}^{D}(z) e^{m \alpha}(w) \sim m e^{m \alpha}(w) \cdot \frac{z}{z^{2}-w^{2}} .
$$

The commutation relations for $e^{\alpha}(z)$ and $e^{-\alpha}(z)$ thus are:

$$
\left[h(z), e^{ \pm \alpha}(w)\right]= \pm\left(i_{z, w}-i_{w, z}\right) \frac{z}{z^{2}-w^{2}} \cdot e^{ \pm \alpha}(w)= \pm \frac{1}{2}(\delta(z-w)+\delta(z+w)) e^{ \pm \alpha}(w) .
$$

From the standard vertex operator calculations this commutation relations immediately imply the exponential operator formulas

$$
\begin{gathered}
e_{D}^{-\alpha}(z)=e^{-\alpha}(z)=\exp \left(-\sum_{n \geq 1} \frac{h_{-n}}{n} z^{2 n}\right) \exp \left(\sum_{n \geq 1} \frac{h_{n}}{n} z^{-2 n}\right) e^{-\alpha} z^{-2 \partial_{\alpha}}, \\
e_{D}^{\alpha}(z)=e^{\alpha}(z)=\exp \left(\sum_{n \geq 1} \frac{h_{-n}}{n} z^{2 n}\right) \exp \left(-\sum_{n \geq 1} \frac{h_{n}}{n} z^{-2 n}\right) e^{-\alpha} z^{2 \partial_{\alpha}} .
\end{gathered}
$$

Note that both of these are entirely even in the variable $z$ operators, i.e., $e_{D}^{-\alpha}(z)=e_{D}^{-\alpha}(-z)$ and $e_{D}^{\alpha}(z)=$ $e_{D}^{\alpha}(-z)$, which is of course consistent with the relations $\mathcal{R}_{\mathcal{D}}\left(T e^{-\alpha}=e^{-\alpha}\right.$ and $\left.T e^{\alpha}=e^{\alpha}\right)$. Note that

$$
e_{D}^{\alpha}(z)=e_{A}^{\alpha}\left(z^{2}\right), \quad e_{D}^{-\alpha}(z)=e_{A}^{-\alpha}\left(z^{2}\right),
$$

where the operators $e_{A}^{\alpha}(z)$ and $e_{A}^{-\alpha}(z)$ describe the boson-fermion correspondence of type A. This is a very interesting occurrence, and is discussed also [RT12] from the point of view of the "fermionization". One should note though that an isomorphism of the spaces of states as Heisenberg modules and the "change of variables" formula (V.51) do not in imply an isomorphism as twisted vertex algebras - these two vertex algebras have a different set of singularities in the OPEs. The equivalence, as [RT12] notes, is as CAR algebras.

We see that the boson-fermion correspondence of type D-A identifies

$$
\phi^{D}(z)=e_{D}^{-\alpha}(z)+z e_{D}^{\alpha}(z), \quad(T \phi)^{D}(z)=e_{D}^{-\alpha}(z)-z e_{D}^{\alpha}(z) .
$$

Here again we see the "shifts" allowed in an isomorphism of vertex algebras.

Thus we have shown that the pair $\left(L_{1} / \mathcal{R}_{\mathcal{D}}, r_{z, w}\left(e^{\alpha} \otimes e^{\alpha}\right)=z^{2}-w^{2}\right)$ describes the twisted vertex algebra which is the bosonic side of the boson-fermion correspondence of type D-A. To summarize all these considerations we have:

Theorem V.10 The boson-fermion correspondence of type D-A is an isomorphism between two twisted vertex algebras: the fermionic side, which is the twisted vertex algebra based on the pair $\left(\mathbb{C}\{\phi\}, r^{D_{f}}\right)$; and the bosonic side, which is the twisted vertex algebra based on the pair $\left(\mathbb{C}[\mathbb{Z} \alpha] / \mathcal{R}_{\mathcal{D}}, r^{D_{b}}\right)$. 
In order to prove Lemma III.21 we need to compare the vacuum expectation values on the bosonic side with those on the fermionic side. We need to take into account the isomorphism formula (V.52) if we are to apply Proposition V.6.

$$
\begin{gathered}
\left\langle 0\left|\phi^{D}\left(z_{1}\right) \phi^{D}\left(z_{2}\right) \ldots \phi^{D}\left(z_{2 n}\right)\right| 0\right\rangle=\left\langle 0\left|\left(e_{D}^{-\alpha}\left(z_{1}\right)+z_{1} e_{D}^{\alpha}\left(z_{1}\right)\right)\left(e_{D}^{-\alpha}\left(z_{2}\right)+z_{2} e_{D}^{\alpha}\left(z_{2}\right)\right) \ldots\left(e_{D}^{-\alpha}\left(z_{2 n}\right)+z_{2 n} e_{D}^{\alpha}\left(z_{2 n}\right)\right)\right| 0\right\rangle \\
=\left\langle 0\left|e^{-\alpha}\left(z_{1}\right) e^{-\alpha}\left(z_{2}\right) \ldots e^{-\alpha}\left(z_{2 n}\right)\right| 0\right\rangle+\sum_{i=1}^{2 n} z_{i}\left\langle 0\left|e^{-\alpha}\left(z_{1}\right) \ldots e^{\alpha}\left(z_{i}\right) \ldots e^{-\alpha}\left(z_{2 n}\right)\right| 0\right\rangle \\
\quad+\sum_{i<j}^{2 n} z_{i} z_{j}\left\langle 0\left|e^{-\alpha}\left(z_{1}\right) \ldots e^{\alpha}\left(z_{i}\right) \ldots e^{\alpha}\left(z_{j}\right) \ldots e^{-\alpha}\left(z_{2 n}\right)\right| 0\right\rangle+\ldots \\
\quad+\sum_{i_{1}<i_{2} \ldots<i_{k}}^{2 n} z_{i_{1}} z_{i_{2}} \ldots z_{i_{k}}\left\langle 0\left|e^{-\alpha}\left(z_{1}\right) \ldots e^{\alpha}\left(z_{i_{1}}\right) \ldots e^{\alpha}\left(z_{i_{k}}\right) \ldots e^{-\alpha}\left(z_{2 n}\right)\right| 0\right\rangle+\ldots
\end{gathered}
$$

The factor of $\delta_{m_{1}+m_{2}+\cdots+m_{n}, 0}$ in the right-hand side of the Proposition $\nabla .6$ forces all the sums but one to vanish: the only remaining sum has the product of exactly $n$ factors of $z_{i_{k}}$ in it, as it will have exactly $n e^{\alpha}$ 's and as many $e^{-\alpha}$ 's in it. Denote in this non-vanishing sum the indexes corresponding to $e^{-\alpha}$ by $z_{j_{k}}$. Hence we have a disjoint split $\{1,2, \ldots 2 n\}=\left\{i_{1}, i_{2}, \ldots, i_{n}\right\} \sqcup\left\{j_{1}, j_{2}, \ldots, j_{n}\right\}$ and we can write the non-vanishing sum as

$$
\sum_{i_{1}<i_{2} \cdots<i_{n}}^{2 n} z_{i_{1}} z_{i_{2}} \cdots z_{i_{n}}\left\langle 0\left|e^{-\alpha}\left(z_{j_{1}}\right) e^{\alpha}\left(z_{i_{1}}\right) e^{-\alpha}\left(z_{j_{2}}\right) e^{\alpha}\left(z_{i_{2}}\right) \ldots e^{-\alpha}\left(z_{j_{n}}\right) e^{\alpha}\left(z_{i_{n}}\right)\right| 0\right\rangle .
$$

From Proposition V.6 and $r_{z_{i}, z_{j}}\left(e^{\alpha} \otimes e^{-\alpha}\right)=r_{z_{i}, z_{j}}\left(e^{-\alpha} \otimes e^{\alpha}\right)=\frac{1}{z_{i}^{2}-z_{j}^{2}}$

$$
\left\langle 0\left|\phi^{D}\left(z_{1}\right) \phi^{D}\left(z_{2}\right) \ldots \phi^{D}\left(z_{2 n}\right)\right| 0\right\rangle=i_{z} \frac{\sum_{i_{1}<i_{2} \cdots<i_{n}}^{2 n} z_{i_{1}} z_{i_{2}} \cdots z_{i_{n}} \prod_{k<l}^{n}\left(z_{i_{k}}^{2}-z_{i_{l}}^{2}\right) \prod_{p<q}^{n}\left(z_{j_{p}}^{2}-z_{j_{q}}^{2}\right)}{\prod_{k, p}^{n} z_{i_{k}}^{2}-z_{j_{p}}^{2}} .
$$

Thus since the boson-fermion correspondence identifies the fields $\phi^{D}(z)=e_{D}^{-\alpha}(z)+z e_{D}^{\alpha}(z)$, lemma $\llbracket 1.21$ follows directly as the Pfaffian equality for the vacuum expectation values on the fermionic side is a special case of Proposition V.1. It is important that this Pfaffian identity follows directly from the correspondence of type $\mathrm{D}$, and is a representative "imprint" of the boson-fermion correspondence.

\section{Boson-fermion correspondence of type D-A and order $N$}

In this section we briefly show how the boson-fermion correspondence of type D-A extends to order $N$ (and thereby give two examples of twisted vertex algebra of order $N$ ). Similarly to section $V D$ we are working with a space of fields $V=H_{T}^{N}(\mathbb{C}\{\phi\})$, and a space of states $W=H_{D}(\mathbb{C}\{\phi\})$, but now we allow $N$ to be any natural number, $N \geq 2$. The projection map (recall definition IV.15) is again the algebra homomorphism defined by $\pi_{T}\left(T^{i} \phi\right)=\phi$, for any $0 \leq i \leq N-1$. As in Section VD the bicharacter $r^{D}: V \otimes V \rightarrow \mathbf{F}^{N}(z, w)^{+, w}$ is defined by $r_{z, w}^{D}(\phi \otimes \phi)=\frac{1}{z-w}$. Theorem IV.41 guarantees that the pair $\left(\mathbb{C}\{\phi\}, r_{z, w}^{D}\right)$ will generate a twisted vertex algebra of order $N$. In the twisted vertex algebra we have the following descendant fields $T^{i} \phi^{D}(z)=\phi^{D}\left(\epsilon^{i} z\right)$, for any $0 \leq i \leq N-1$, with OPEs

$$
T^{i} \phi^{D}(z) T^{j} \phi^{D}(w) \sim \frac{1}{\epsilon^{i} z-\epsilon^{j} w} .
$$

The boson-fermion correspondence is determined via the Heisenberg element

$$
h=\frac{1}{N} \sum_{i=0}^{N-1} \epsilon^{i-1}\left(T^{i-1} \phi\right)\left(T^{i} \phi\right) .
$$

We have $T h=\epsilon^{-1} h$, hence we index the field $h(z):=Y(h, z)=\sum_{n \in \mathbb{Z}} h_{n} z^{-N n-1}$. Lemma III.22 then holds immediately. Denote

$$
e_{\phi}^{\alpha}(w)=\frac{1}{N}\left(\sum_{i=0}^{N-1} \epsilon^{-i} T^{i} \phi^{D}(w)\right), \quad e_{\phi}^{\epsilon^{k} \alpha}(w)=\frac{1}{N}\left(\sum_{i=0}^{N-1} \epsilon^{(k-1) i} T^{i} \phi^{D}(w)\right) .
$$


We get

$$
e_{\phi}^{\epsilon^{k} \alpha}(z)=\exp \left(\epsilon^{-k} \sum_{n \geq 1} \frac{h_{-n}}{n} z^{N n}\right) \exp \left(\epsilon^{k} \sum_{n \geq 1} \frac{h_{n}}{n} z^{-N n}\right) U_{\epsilon^{k} \alpha}(z),
$$

where $U_{\alpha}(z)$ acts as a constant on each Heisenberg submodule, $U_{\epsilon^{k} \alpha}(z)=e_{\phi}^{\epsilon^{k} \alpha} z^{1-k+N \partial_{\alpha}}$, and $e_{\phi}^{\epsilon^{k} \alpha}$ is the highest weight vector of the Heisenberg submodule. This establishes the boson-fermion correspondence of order $N$.

\section{Other examples of twisted vertex algebras; the correspondence of type C}

There are other important examples in the literature of boson-fermion (or boson-boson) correspondences which can be shown to be isomorphisms of twisted vertex algebras: in particular the CKP correspondence (also called correspondence of type C, DJKM81b] and [vOS11]), and the "super boson-fermion correspondence of type B" ([KvdL89]), are both isomorphisms of twisted vertex algebras.

Lemma V.11 The correspondence of type $C$ is an isomorphism of twisted vertex algebras, where one of the sides is a twisted vertex algebra based on the pair $\left(\mathbb{C}[h], r^{C}\right)$ via Theorem IV.41.

Proof: The "left-hand side" of the CKP correspondence has a space of states $V=H_{T}^{2}(\mathbb{C}[h]$ ) (recall the free Leibnitz module $H_{T}^{2}(\mathbb{C}[h])$ of example IV.9), and the twisted vertex algebra is generated by the specific bicharacter value

$$
r_{z, w}^{C}(h \otimes h)=\frac{1}{z+w} .
$$

The OPE for the corresponding field $h_{\phi}(z)$ directly follows from the fact that $h_{\phi}$ is primitive:

$$
h_{\phi}(z) h_{\phi}(w) \sim \frac{1}{z+w} \sim h_{\phi}(w) h_{\phi}(z) .
$$

We use $\phi_{j}$ as notation for the modes of $h_{\phi}(z)$ to follow [vOS11]. The field $h_{\phi}(z)$ is indexed as follows: $h_{\phi}(z)=\sum_{j \in \mathbb{Z}+1 / 2} \phi_{j} z^{j-1 / 2}$. Theorem IV.41 guarantees that the pair $\left(\mathbb{C}[h], r_{z, w}^{C}\right)$ will generate a twisted vertex algebra of order 2 .

Other examples of twisted vertex algebras are supplied by the representation theory of affine Lie algebras and affine Lie super algebras, see for example [Fre80], FF85], [FFR91] and [KWY98].

\section{SUMMARY}

There are three main results of this paper: first, we derived the new boson-fermion correspondence of type D-A. Second, we defined the new concept of a twisted vertex algebra of order $N$, which generalize supervertex algebras (in the sense that a super vertex algebra is a special case of twisted vertex algebras of order 1). This new concept of a twisted vertex algebra was designed to answer the following question "What is a boson-fermion correspondence - isomorphism of what mathematical structures?". As a technical set of tools we developed the bicharacter construction which provided us with a general way of producing examples of twisted vertex algebras. We proved formulas for the OPEs, analytic continuations, normal ordered products and vacuum expectation values using the underlying Hopf algebra structure and the bicharacter construction. Finally, we proved that the correspondences of types B, C and D-A are isomorphisms of twisted vertex algebras.

[ACJ13] Iana I. Anguelova, Ben Cox, and Elizabeth Jurisich. N-point locality for vertex operators: normal ordered products, operator product expansions, twisted vertex algebras. arxiv, submitted, 2013.

[Ang08] Iana I. Anguelova. Super-bicharacter construction of $H_{D}$-quantum vertex algebras. Rep. Math. Phys., 61(2):253-263, 2008.

[Ang13] Iana Anguelova. Boson-fermion correspondence of type B and twisted vertex algebras. In Proceedings of the 9-th International Workshop "Lie Theory and Its Applications in Physics" (LT-9), Varna, Bulgaria, Springer Proceedings in Mathematics and Statistics, 2013. 
[BFFO04] Christian Brouder, Bertfried Fauser, Alessandra Frabetti, and Robert Oeckl. Quantum field theory and Hopf algebra cohomology. J. Phys. A, 37(22):5895-5927, 2004.

[BK04] Bojko Bakalov and Victor G. Kac. Twisted modules over lattice vertex algebras. In Lie theory and its applications in physics V, pages 3-26. World Sci. Publ., River Edge, NJ, 2004.

[Bor86] Richard E. Borcherds. Vertex algebras, Kac-Moody algebras, and the Monster. Proc. Nat. Acad. Sci. U.S.A., 83(10):3068-3071, 1986.

[Bor01] Richard E. Borcherds. Quantum vertex algebras. In Taniguchi Conference on Mathematics Nara '98, volume 31 of Adv. Stud. Pure Math., pages 51-74, Tokyo, 2001. Math. Soc. Japan.

[DJKM81a] Etsurō Date, Michio Jimbo, Masaki Kashiwara, and Tetsuji Miwa. Transformation groups for soliton equations. III. Operator approach to the Kadomtsev-Petviashvili equation. J. Phys. Soc. Japan, 50(11):3806$3812,1981$.

[DJKM81b] Etsurō Date, Michio Jimbo, Masaki Kashiwara, and Tetsuji Miwa. Transformation groups for soliton equations. VI. KP hierarchies of orthogonal and symplectic type. J. Phys. Soc. Japan, 50(11):3813-3818, 1981.

[DJKM82] Etsurō Date, Michio Jimbo, Masaki Kashiwara, and Tetsuji Miwa. Transformation groups for soliton equations. IV. A new hierarchy of soliton equations of KP-type. Phys. D, 4(3):343-365, 1981/82.

[FBZ04] Edward Frenkel and David Ben-Zvi. Vertex algebras and algebraic curves, volume 88 of Mathematical Surveys and Monographs. American Mathematical Society, Providence, RI, second edition, 2004.

[FF85] Alex J. Feingold and Igor B. Frenkel. Classical affine algebras. Adv. in Math., 56(2):117-172, 1985.

[FFR91] Alex J. Feingold, Igor B. Frenkel, and John F. X. Ries. Spinor construction of vertex operator algebras, triality, and $E_{8}^{(1)}$, volume 121 of Contemporary Mathematics. American Mathematical Society, Providence, RI, 1991.

[FHL93] Igor Frenkel, Yi-Zhi Huang, and James Lepowsky. On axiomatic approaches to vertex operator algebras and modules. Mem. Amer. Math. Soc., 104(494):viii+64, 1993.

[FLM88] Igor Frenkel, James Lepowsky, and Arne Meurman. Vertex operator algebras and the Monster, volume 134 of Pure and Applied Mathematics. Academic Press Inc., Boston, MA, 1988.

[FR97] E. Frenkel and N. Reshetikhin. Towards Deformed Chiral Algebras. In Proceedings of the Quantum Group Symposium at the XXIth International Colloquium on Group Theoretical Methods in Physics, Goslar 1996, pages 6023-+, 1997.

[Fre80] Igor B. Frenkel. Spinor representations of affine Lie algebras. Proc. Nat. Acad. Sci. U.S.A., 77(11, part 1):6303-6306, 1980.

[Fre81] Igor B. Frenkel. Two constructions of affine Lie algebra representations and boson-fermion correspondence in quantum field theory. J. Funct. Anal., 44(3):259-327, 1981.

[Kac90] Victor G. Kac. Infinite-dimensional Lie algebras. Cambridge University Press, Cambridge, third edition, 1990.

[Kac98] Victor Kac. Vertex algebras for beginners, volume 10 of University Lecture Series. American Mathematical Society, Providence, RI, second edition, 1998.

[Kas95] Christian Kassel. Quantum groups, volume 155 of Graduate Texts in Mathematics. Springer-Verlag, New York, 1995.

[KR87] V. G. Kac and A. K. Raina. Bombay lectures on highest weight representations of infinite-dimensional Lie algebras, volume 2 of Advanced Series in Mathematical Physics. World Scientific Publishing Co. Inc., Teaneck, NJ, 1987.

[KvdL89] V. G. Kac and J. W. van de Leur. Super boson-fermion correspondence of type B. In Infinite-dimensional Lie algebras and groups (Luminy-Marseille, 1988), volume 7 of Adv. Ser. Math. Phys., pages 369-406. World Sci. Publ., Teaneck, NJ, 1989.

[KW94] Victor Kac and Weiqiang Wang. Vertex operator superalgebras and their representations. In Mathematical aspects of conformal and topological field theories and quantum groups (South Hadley, MA, 1992), volume 175 of Contemp. Math., pages 161-191. Amer. Math. Soc., Providence, RI, 1994.

[KWY98] Victor G. Kac, Weiqiang Wang, and Catherine H. Yan. Quasifinite representations of classical Lie subalgebras of $W_{1+\infty}$. Adv. Math., 139(1):56-140, 1998.

[Li94] Hai Sheng Li. Symmetric invariant bilinear forms on vertex operator algebras. J. Pure Appl. Algebra, 96(3):279297, 1994.

[Li96] Haisheng Li. Local systems of vertex operators, vertex superalgebras and modules. J. Pure Appl. Algebra, 109(2):143-195, 1996.

[Li06] Haisheng Li. A new construction of vertex algebras and quasi-modules for vertex algebras. Adv. Math., 202(1):232-286, 2006.

[LL04] James Lepowsky and Haisheng Li. Introduction to vertex operator algebras and their representations, volume 227 of Progress in Mathematics. Birkhäuser Boston Inc., Boston, MA, 2004.

[MJD00] T. Miwa, M. Jimbo, and E. Date. Solitons: differential equations, symmetries and infinite dimensional algebras. Cambridge tracts in mathematics. Cambridge University Press, 2000.

[RT12] Karl-Henning Rehren and Gennaro Tedesco. Multilocal fermionization. Letters in Mathematical Physics, pages 1-18, September 2012.

[vdLO09] J. W. van de Leur and A. Yu. Orlov. Random turn walk on a half line with creation of particles at the origin. Phys. Lett. A, 373(31):2675-2681, 2009.

[vOS11] J. W. van de Leur, A. Y. Orlov, and T. Shiota. CKP Hierarchy, Bosonic Tau Function and Bosonization 
Formulae. ArXiv e-prints, February 2011.

[Wak01] Minoru Wakimoto. Lectures on infinite-dimensional Lie algebra. World Scientific Publishing Co. Inc., River Edge, NJ, 2001.

[Wan99] Weiqiang Wang. Dual pairs and infinite dimensional Lie algebras. In Recent developments in quantum affine algebras and related topics (Raleigh, NC, 1998), volume 248 of Contemp. Math., pages 453-469. Amer. Math. Soc., Providence, RI, 1999.

[Xu98] Xiaoping Xu. Introduction to vertex operator superalgebras and their modules, volume 456 of Mathematics and its Applications. Kluwer Academic Publishers, Dordrecht, 1998.

[You89] Yuching You. Polynomial solutions of the BKP hierarchy and projective representations of symmetric groups. In Infinite-dimensional Lie algebras and groups (Luminy-Marseille, 1988), volume 7 of Adv. Ser. Math. Phys., pages 449-464. World Sci. Publ., Teaneck, NJ, 1989. 